\title{
Advanced Image Deconvolution Techniques for Super-resolution Microscopy
}

\author{
Shun Qin
}

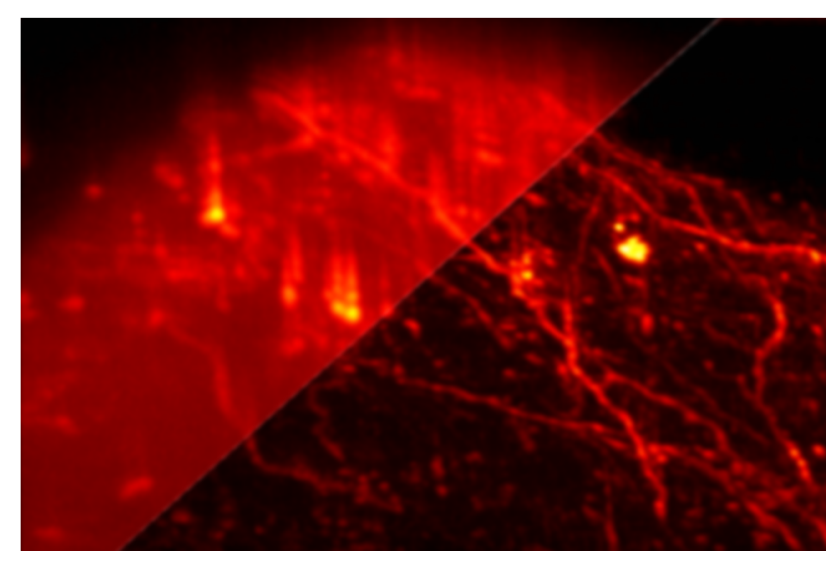





\title{
Advanced Image Deconvolution Techniques for Super-resolution Microscopy
}

\author{
Dissertation \\ for the award of the degree \\ "Doctor rerum naturalium" \\ of the Georg-August-Universität Göttingen \\ within the doctoral program \\ Physics of Biological and Complex Systems \\ of the Göttingen Graduate School of Neurosciences, Biophysics, and \\ Molecular Biosciences (GGNB) \\ of the Georg-August University School of Science (GAUSS)
}

\author{
submitted by \\ Shun Qin \\ from Guigang, China
}

Göttingen, July 2019 


\section{Thesis Committee}

Prof. Dr. Jörg Enderlein

Third Institute of Physics-Biophysics

Georg-August-Universität Göttingen

Dr. Michael Habeck

Institute for Mathematical Stochastics

Group Georg-August-Universität Göttingen

Prof. Dr. Ulrich Parlitz

Biomedical Physics

Max Planck Institute for Dynamics and Self-Organisation, Göttingen

\section{Members of the Examination Board}

Referee: Prof. Dr. Jörg Enderlein

Third Institute of Physics-Biophysics

Georg-August-Universität Göttingen

$2^{\text {nd }}$ Referee: Prof. Dr. Ulrich Parlitz

Biomedical Physics Group

Max Planck Institute for Dynamics and Self-Organisation, Göttingen

\section{Further members of the Examination Board}

Dr. Michael Habeck

Institute for Mathematical Stochastics

Georg-August-Universität Göttingen

Prof. Dr. Markus Zweckstetter

Protein structure determination using NMR

Max Planck Institute for Biophysical Chemistry

Dr. Florian Rehfeldt

Third Institute of Physics-Biophysics

Georg-August-Universität Göttingen

Prof. Dr. Jens Frahm

Biomedical NMR

Max Planck Institute for Biophysical Chemistry

Date of oral examination: 10.09.2019 


\section{CONTENTS}

\section{Introduction of Super-Resolution Fluorescence Microscopy 1}

1.1 Fluorescence Microscopy ............................................................................... 1

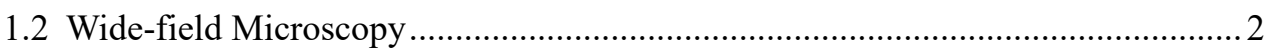

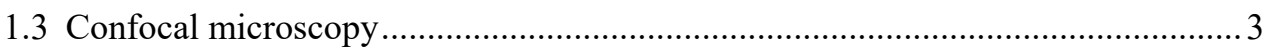

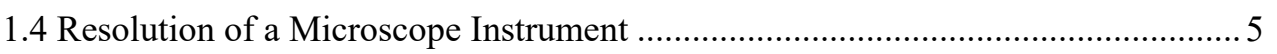

1.5 Overview of the state-of-the-art super-resolution microscopies …………............ 5

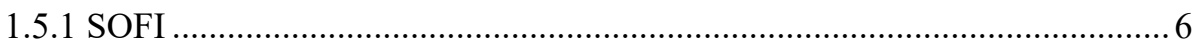

1.5.2 Structured Illumination Microscopy ........................................................

1.5.3 Image Scanning Microscopy ……........................................................ 7

1.5.4 Deconvolution for Super-Resolution Microscopy Image Restoration........... 8

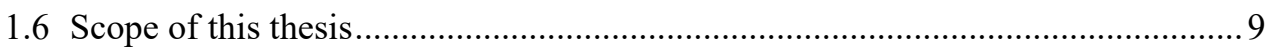

2 Super-resolution Optical Fluctuation Imaging $\quad 10$

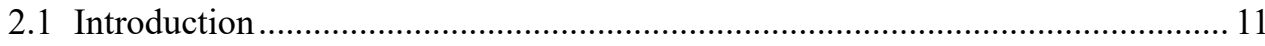

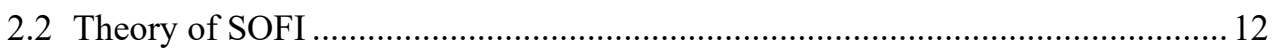

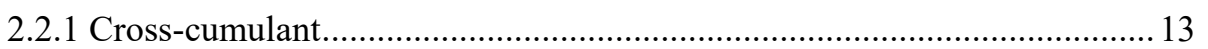

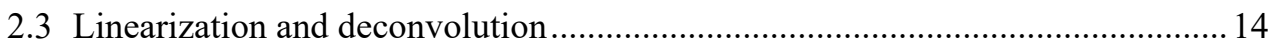

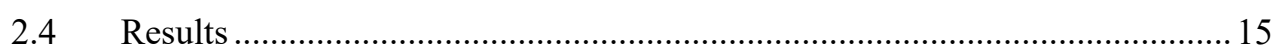

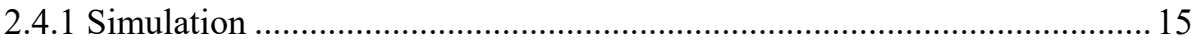

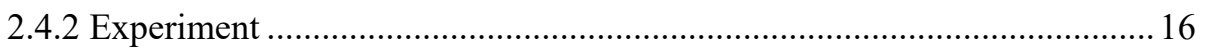

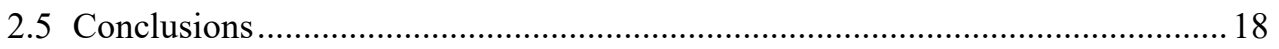

3 Spinning Disk Confocal-Image Scanning Microscopy 18

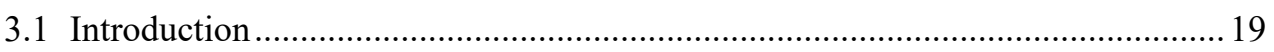

3.2 Theory of Image Scanning Microscopy ………................................................ 20

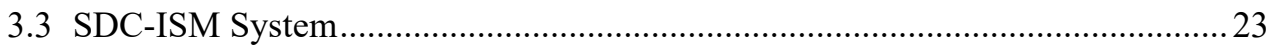

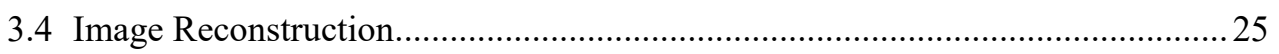

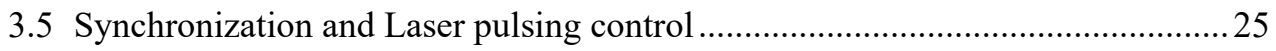

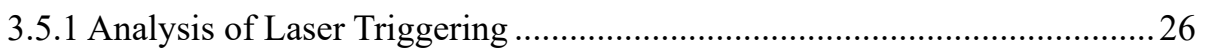

3.5.2 Implementation on a Field Programmable Gate Arrays (FPGA) ............... 28

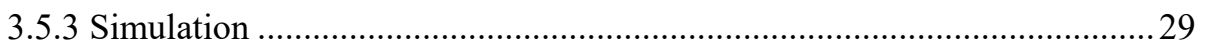

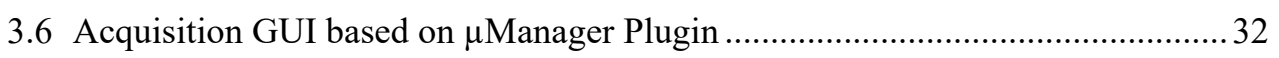

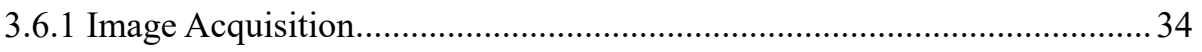

3.6.2 Image Reconstruction Software ………………….................................... 35

3.7 Confocal Light Spots Detection and Localization ................................................... 37

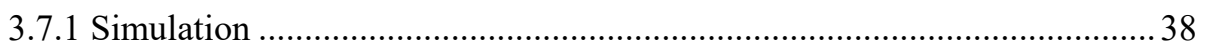

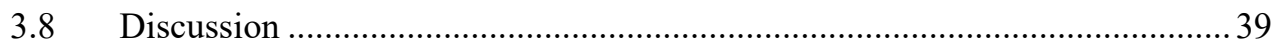

4 Accurate Gaussian Fitting 39

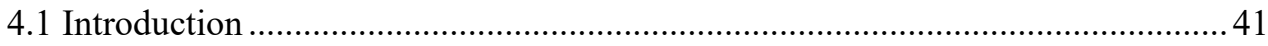

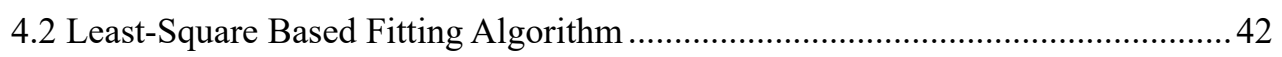

4.3 Maximum Likelihood Estimation Based Gaussian Fitting Algorithm ................. 45

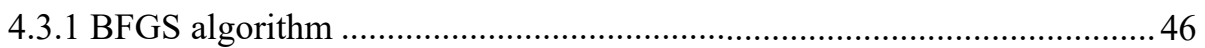




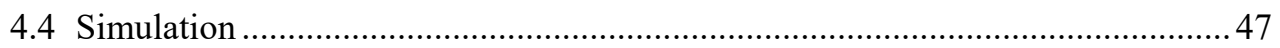

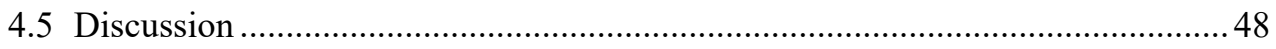

5 Simulation of Structured Illumination Microscopy 47

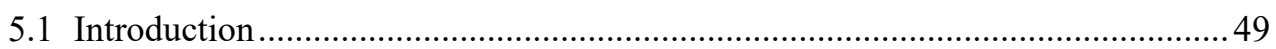

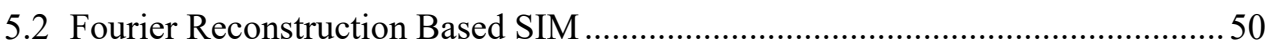

5.2.1 Calculate Frequency and Phase of the Modulation Pattern..........................53

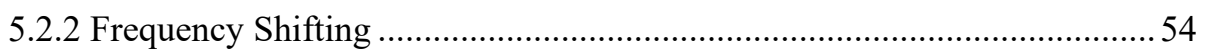

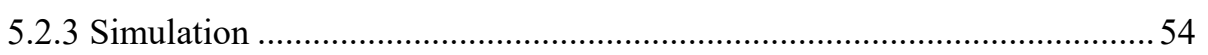

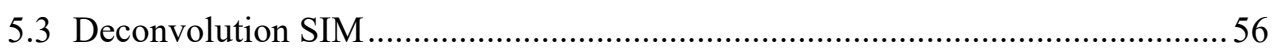

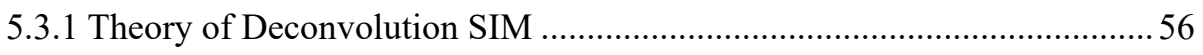

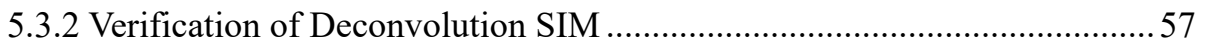

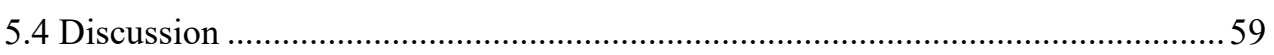

6 Solving L1-Norm Regularized Problems Based on Non-linear Optimization 58

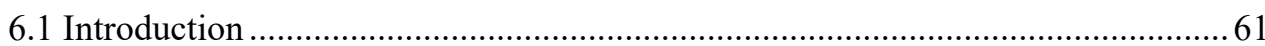

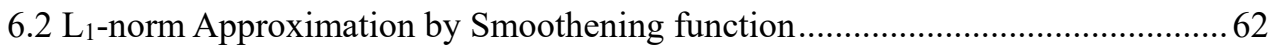

6.3 Compressed Sensing Reconstruction Problem...................................................... 63

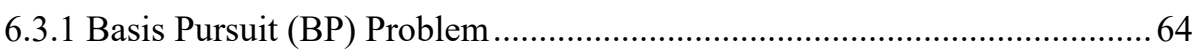

6.3.2 Fourier Measured Compressed Sensing Reconstruction Problem............... 64

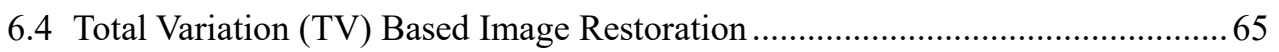

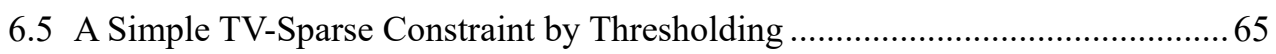

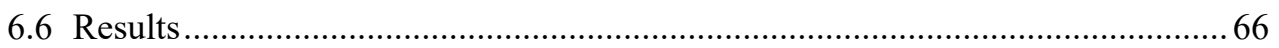

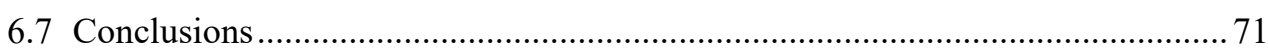

7 Artifact Removal Deconvolution $\quad 69$

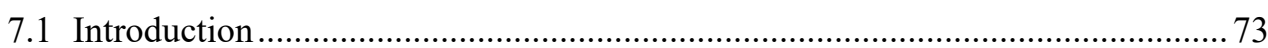

7.2 Concept of Deconvolution and some Basic Deconvolution Methods ................... 74

7.3 Maximum a Posteriori (MAP) Estimation Based Deconvolution with

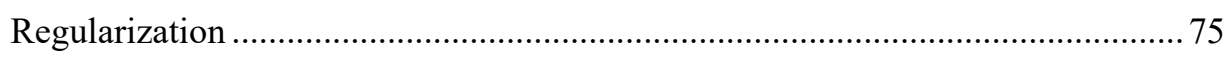

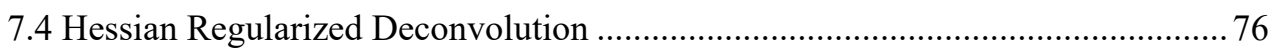

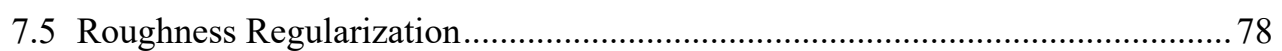

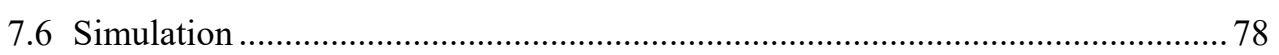

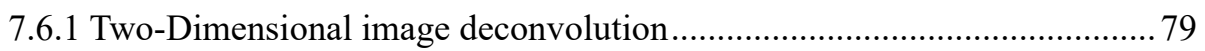

7.6.2 Three-Dimensional image deconvolution.................................................... 81

7.7 Artifact Removal Deconvolution with Hessian Regularization ................................ 82

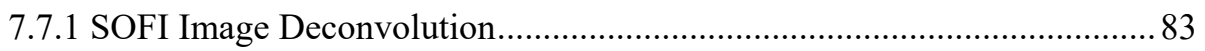

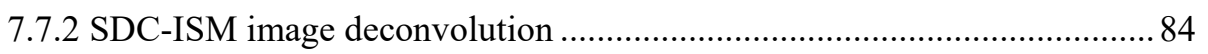

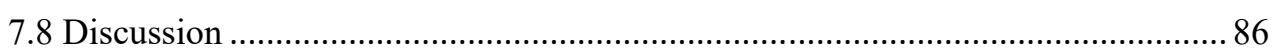

8 Airy Beam Light-Sheet Microscopy $\quad 83$

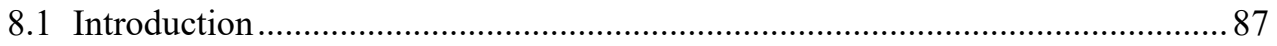

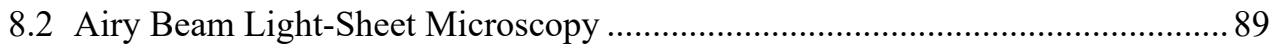

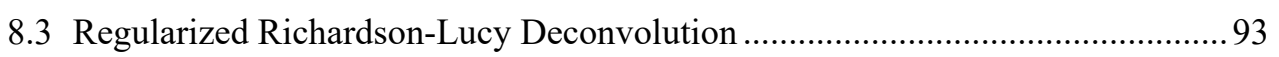

8.3.1 Richardson-Lucy Deconvolution with TV regularization (RLTV)............. 93

8.3.2 Richardson-Lucy Deconvolution with Roughness Regularization (RLRF)94 


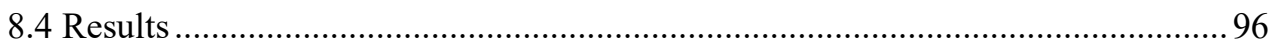

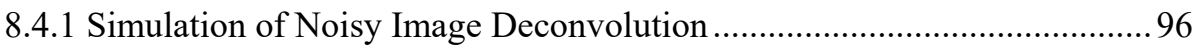

8.4.2 Deconvolution of Real Sample Image ........................................................... 97

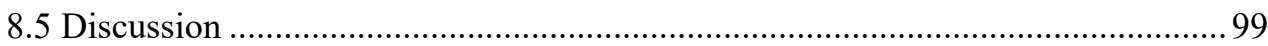

9 Discussion and Outlook 101

$\begin{array}{lr}\text { BIBLIOGRAPHY } & 103\end{array}$

$\begin{array}{ll}\text { ACKNOWLEDGEMENTS } & 111\end{array}$ 



\section{Chapter $1 \quad$ Introduction of Super-Resolution Fluorescence} Microscopy

\subsection{Fluorescence Microscopy}

Fluorescence microscopy is an essential technique for modern biological research to explore function and dynamics of proteins, cells, and tissue [1-3]. The principle of fluorescence microscopy is based on the physical property of fluorophores to absorb light at specific wavelengths and to emit this absorbed energy as fluorescence at longer wavelengths. This process of excitation and emission of a fluorophore [4] is depicted in figure 1.1. An electron in the fluorophore's ground state absorbs a photon with energy $\mathrm{hv}_{1}$ and jumps into a higher electronic state. From there, it spontaneously returns to the ground state while emitting a fluorescence photon with lower energy and larger wavelength.

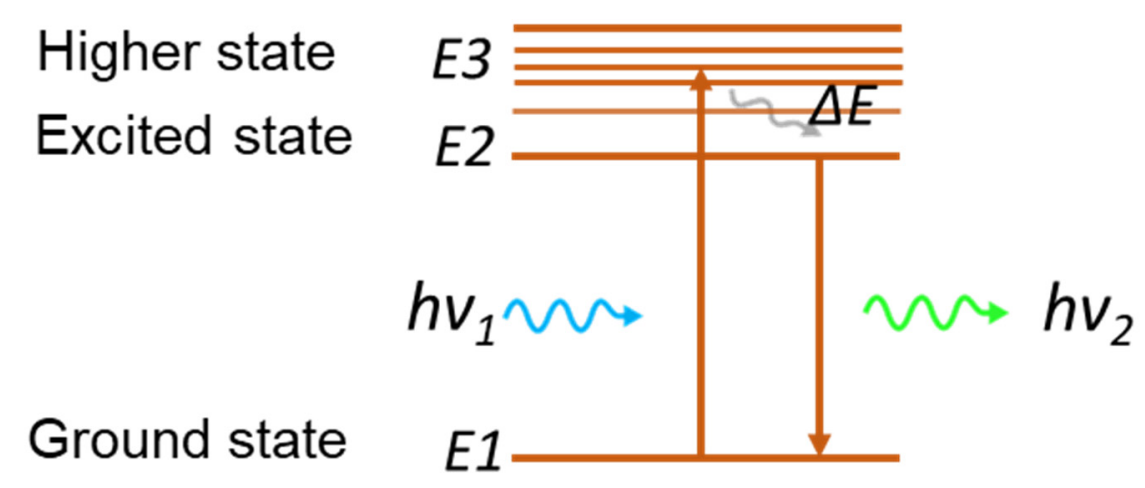

Figure 1.1

In fluorescence microscopy, one employs fluorophores as labels of specific molecules or structures in cells, using special antibodies or other chemical coupling protocols. These fluorescent labels then emit fluorescence when illuminated by excitation light. Because only the labeled molecules or structure in the sample do generate fluorescence, only the structures of interest are visible in a microscopy image, if one uses correct spectral filters that transmit the fluorescent light but reject the excitation light. This fluorescence is typically recorded a camera that is sensitive to fluorescence light. So far, wide-field microscopy and confocal microscopy are the two most widely used fluorescence microscopies. 


\subsection{Wide-field Microscopy}

Figure 1.2 shows the principle of wide-field microscopy [5]. The excitation light firstly goes through an excitation filter, is then reflected by a dichroic mirror, and is finally focused on the sample by an objective. When the excitation light is on, the fluorophores in the sample emit fluorescence, which is collected through the same objective as used for excitation (epi-fluorescence setup), then passes the dichroic mirror, and is finally imaged by a tube lens onto a camera, which records the image.

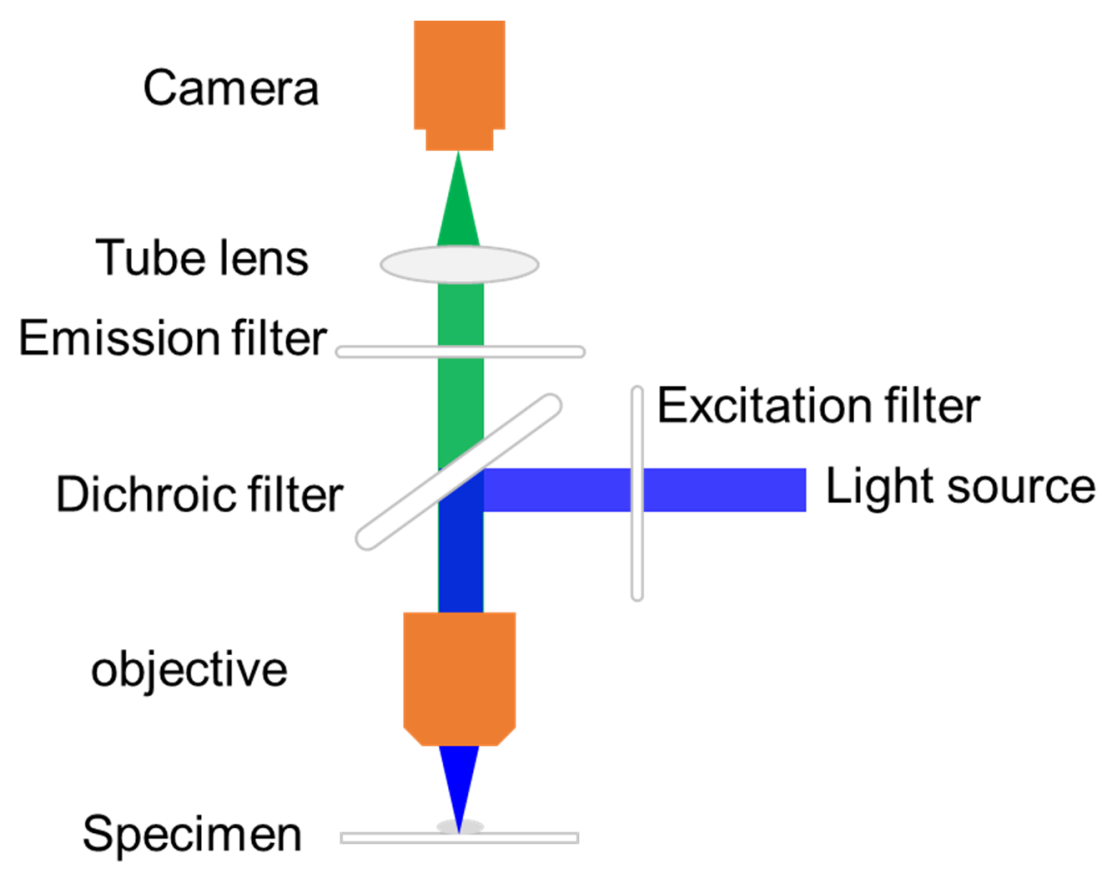

Figure 1.2

Wide-field microscopy has the advantage to be fast, because an entire field of view in the sample is illuminated and imaged instantaneously. However, a wide-field microscope captures all the fluorescence that is generated throughout the whole volume of a sample, so that it does not have so-called z-sectioning capability and can thus not easily be used to record three-dimensional images of a sample.

It is simpler to understand the performance of a wide-field microscope via its Point Spread Function (PSF) and Optical Transfer Function (OTF). The 3D PSF of a widefield microscope is shown in Figure 1.3. It shows that the resolution along the optical axis ( $z$-direction) of a wide-field microscope is worse than its lateral resolution. The $3 \mathrm{D}$ OTF shows the missing cones of frequencies around the optical axis, which means that information of spatial frequencies along the $z$-direction is not transmitted, so that there is no axial information measured by a wide-field microscope. This explain in Fourier domain why the wide-field microscopy does not have the capacity of z-sectioning in 3D imaging. 

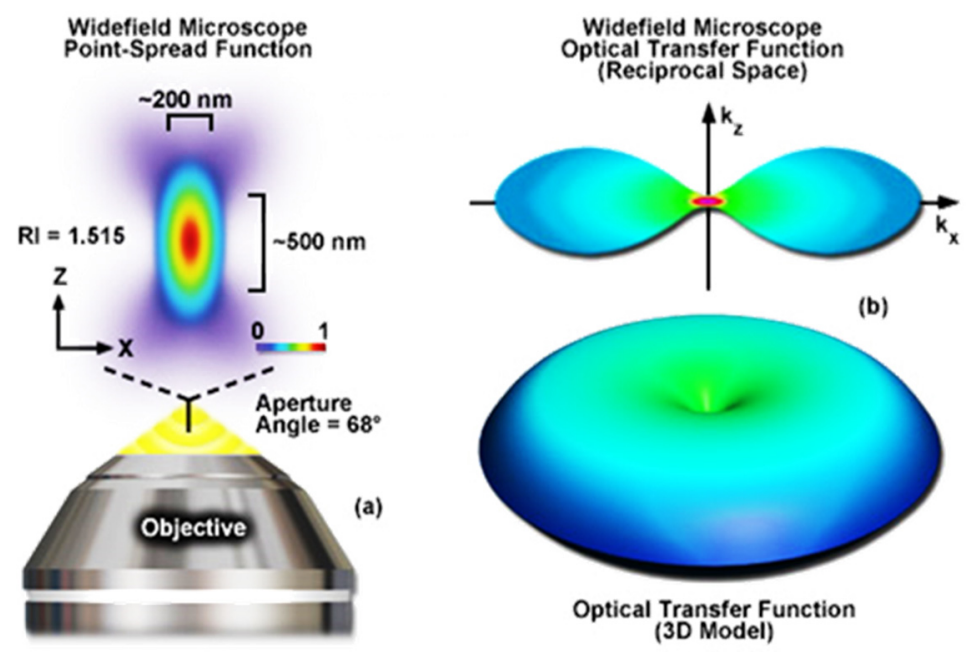

Figure 1.3 PSF and OTF of wide-field microscope. Images taken from ref. [6]. Left: PSF, right:

OTF

The imaging function of wide-field microscopy can be simply described by

$$
I(x, y, z)=\iiint U(x-u, y-v, w-z) O(u, v, w) d u d v d w
$$

where $U(x, y, w)$ is the PSF of the imaging system, $O(u, v, w)$ is the object function, $I(x, y, z)$ is the image of slices measured by detector along $z$-direction. Based on this imaging model, deconvolution can be used to improve the image quality of wide-field microscopy.

\subsection{Confocal microscopy}

Confocal microscopy is the second most important fluorescence microscopy, which was is developed specifically for $z$-sectioning (recoding three-dimensional images of a sample) [7]. Compared to wide-field microscopy, confocal microscopy introduces a pinhole before the detector, just as shown in Figure 3. The effect of the pinhole is that the outof-focus light is blocked and cannot be captured by the detector. This property enables confocal microscopy to perform $z$-sectioning. Confocal microscopy is a very important and widely used technique for 3D fluorescence imaging. The $z$-sectioning image is improved significantly compared to a wide-field microscopy image, as the background is removed. Apparently, the PSF of confocal microscopy is also improved compared to wide-field microscopy.

Mathematically, the analysis of the PSF of a confocal microscope is a little bit more complex than that of a wide-field microscope as a pinhole is introduced. The PSF of a confocal microscope is determined by both excitation PSF and detection PSF, which are written herein by $P S F_{E X}(r)$ and $P S F_{D E}(r)$, respectively. Strictly, the excitation PSF and detection PSF are not identical. Again, let us discuss the property of confocal 
microscopy by its OTF.

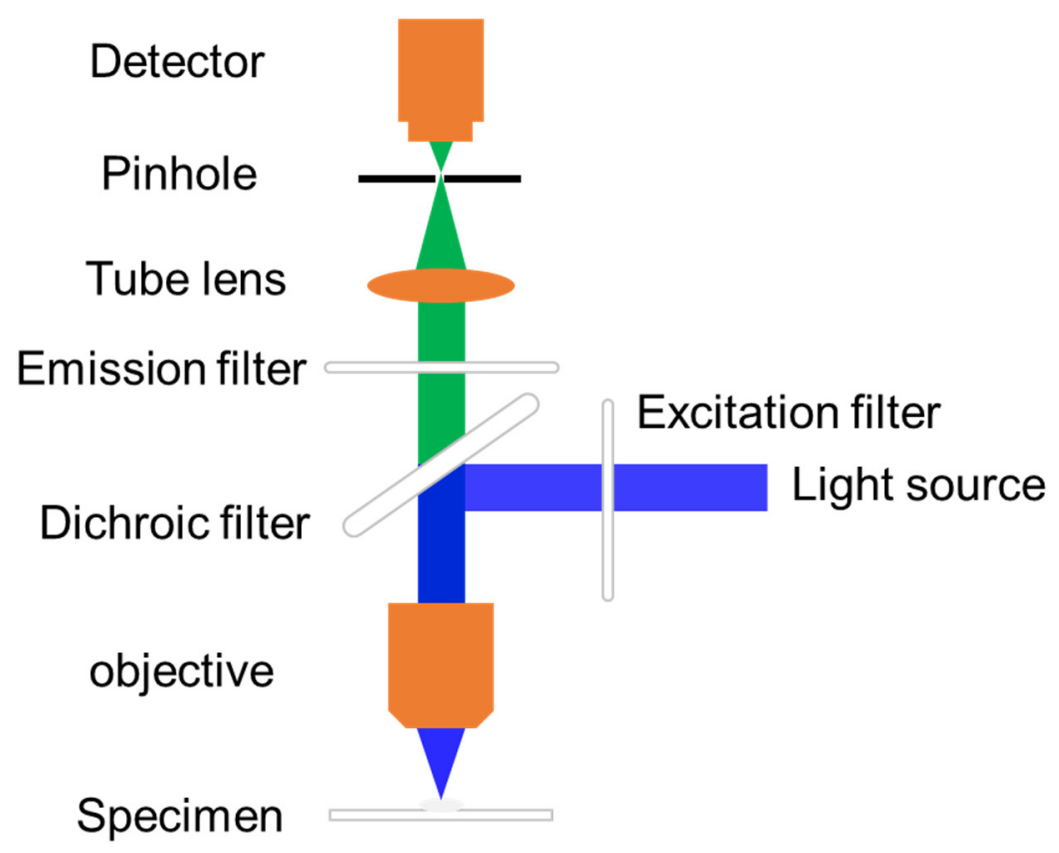

Figure 1.4

With the effect of pinhole, The PSF of confocal microscopy is the product of the excitation PSF and detection PSF, which can be written by

$$
P S F(r)=P S F_{E X}(r) \cdot P S F_{D E}(r)
$$

In practice, the excitation PSF and detection PSF are roughly the same as the PSF of wide-field microscope. According to convolution theorem [8], the multiplication in space domain results in that the OTF of confocal microscope is equal to the convolution between the excitation OTF and the detection OTF. The resulted OTF of confocal microscopy is as Figure 1.5 shows.

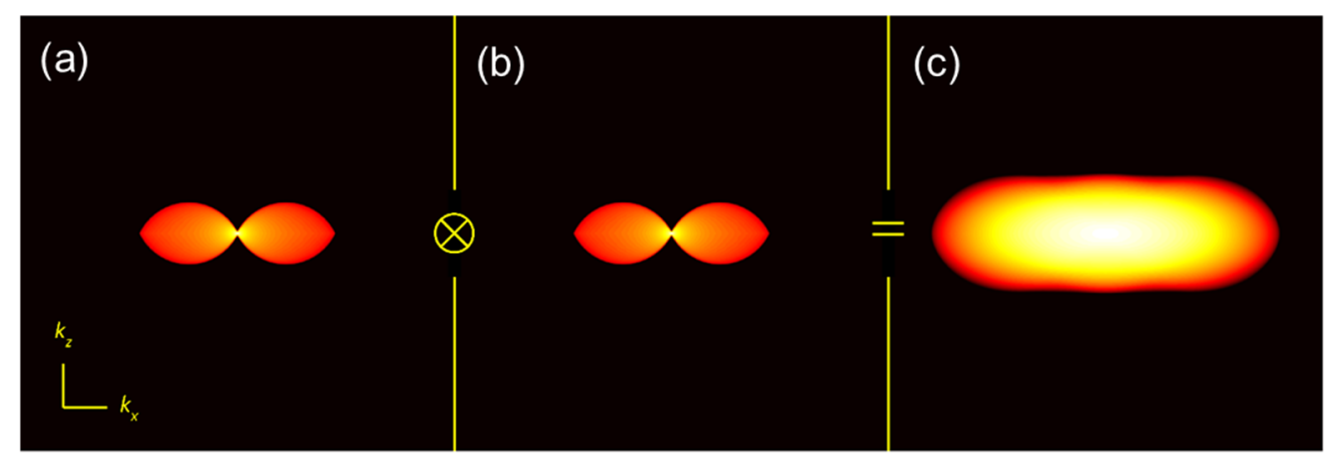

Figure 1.5 confocal microscope OTF (a) Excitation OTF, (b) Detection OTF, (c) convolution between (a) and (b). Images taken from Ref. [9].

The OTF shows that frequencies along the optical axis are now transmitted by the microscope, which means that the confocal microscope can acquire information along the $z$-direction. Therefore, the performance of a confocal microscope in terms of resolution and $z$-sectioning are better than those of wide-field microscopy. However, it results in a poorer signal-to-noise ratio, because the utilization of illumination of excitation light 
is not efficient as the out-of-focus light cannot be used. Besides that, laser scanning slows down imaging speed.

\subsection{Resolution of a Microscope Instrument}

The resolution of a microscope is defined as the smallest distance between two points in the field of view that can still be distinguished by the instrument used. The diffraction-limited resolution of a microscope can be described by Abbe's limit function [10]

$$
\Delta x=\frac{\lambda}{2 N A}
$$

where $\lambda$ is the wavelength of emission light, $N A$ is the numerical aperture which indicates the ability of capacity of collecting light by a lens. Abbe's limit function shows that the diffraction-limited resolution of a microscope is proportional to the imaging wavelength and inversely proportional to the numerical aperture. With Abbe's theory, we can estimate the resolution limit of a modern microscope, which is about $250 \mathrm{~nm}$ laterally, and $500 \mathrm{~nm}$ axially.

State-of-the-art manufacturing technology of microscope optics has brought the optical resolution down to the limit of optical diffraction. In practice, it is very challenge to improve the resolution further by simply improving the $N A$ of an objective when such a limit is closely approached, which creates a bottleneck for traditional optical manufacture technology. Therefore, novel techniques beyond advanced manufacturing technology have to be explored to break the diffraction limit. Nowadays, many super-resolution microscopies have been developed and investigated.

\subsection{Overview of the state-of-the-art super-resolution microscopies}

During the last decade, super-resolution imaging technology has seen its greatest progress in the field of florescence microscopy. The most representative techniques are non-linear super-resolution microscopies such as Stimulated Emission Depletion Microscopy (STED) [11], Photoactivated Localization Microscopy (PALM) [12], Stochastic Optical Reconstruction Microscopy STORM[13], Super-resolution Optical Fluctuation Imaging (SOFI), and linear super-resolution techniques such as 4Pi-microscopy $[14,15]$, Structured Illumination Microscopy (SIM) [16], Image Scanning Microscopy (ISM) $[17,18]$ and some variants of Structured Light-Sheet Microscopy [19].

In practice, non-linear super-resolution are much more powerful in super-resolution enhancement. Many advanced techniques of non-linear super resolution microscopies are based on either stochastic switching (PALM, STORM, SOFI) or fluorescence nonlinearity (STED). These methods either need to measure very large numbers of frames or require very high excitation power. 
In contrast, other types of super-resolution methods that are strictly based on linear fluorescence microscopy are also developed at the same time. Compared to non-linear super-resolution microscopies, linear super-resolution microscopies are less powerful in resolution enhancement. However, linear super-resolution microscopies enable fast imaging and require only low excitation power, which is beneficial by reducing photobleaching during imaging. As mentioned above, there exist many types of linear superresolution microscopies. Here, I only introduce some novel super-resolution techniques that I am going to study in this thesis.

\subsubsection{SOFI}

SOFI is one novel technique to realize super-resolution imaging, which uses a correlation analysis of the temporal intensity fluctuations of emitters for improving the spatial resolution of an image [20]. Compared to other super-resolution imaging technique, such as STED, PALM or STORM, it is a technique that is based on computer calculations rather than complex hardware and/or control techniques [21]. The advantages of SOFI are fast 3D imaging capability, background removal, and easy implementation into a conventional wide-field microscope $[22,23]$.

The principle of SOFI is quite simple. Firstly, SOFI needs a sample labeled with blinking fluorophores, quantum dots for example, which are so small in size that they can be regarded as point objects. The important thing is that their intrinsic emission intensity (brightness) fluctuates randomly and statistically independently from each other. The next step is to take a movie by camera, which records the blinking dynamic of fluorescence over time. Finally, a SOFI image is generated by calculating the temporal correlation of the time-trace in each pixel.

The main process of an implementation of SOFI is the correlation calculation for the time trace of each pixel, which can be easily done with a computer. Actually, it is a statistics-based method that requires very little hardware control. By correlation, it is very easy to remove uncorrelated signals, e.g. background and noise. SOFI take advantage of time-correlation of emitters to enhance resolution, which is determined by the order of correlation. Theoretically, higher order correlations result in larger gains of optical resolution, in principle by a factor of $\sqrt{\mathrm{n}}$ for $\mathrm{n}^{\text {th }}$ order correlation SOFI.

Currently, SOFI has been successfully used for 3D and live-cell multiplane three-dimensional super-resolution imaging [24].

\subsubsection{Structured Illumination Microscopy}

Structured Illumination Microscopy (SIM) is a very powerful and widely used linear super-resolution technique [25]. It is based on conventional wide-field microscopy, but employs a smart illumination technique to double spatial resolution. In fact, the principle of SIM is very simple, and its key aspect is the structured illumination. In theory, any kind of structured illumination can be employed to obtain super-resolution. This 
can be understood by recalling the convolution theorem that states that a multiplication in real space results in a convolution in the Fourier domain. A convolution of two distributions with finite frequency support in Fourier space result in a frequency distribution with extended support. This is the reason why SIM is able to enhance resolution.

The simplest kind of a structured illumination would be a cosine pattern illumination, which is widely used for its simplicity. The Fourier transform of a cosine function is a Dirac function. Therefore, a cosine illumination will shift the Fourier spectrum of the sample function by a value that is equal to the frequency of the cosine function. This allows for measuring higher frequency information of the sample. The power of resolution enhancement of SIM is determined by the frequency of the modeling function. For example, if the frequency of the cosine illumination is just equal to the support boundary of the frequency of the detection OTF, then a full resolution doubling can be obtained by SIM. Except to this super-resolution, the advantages of cosine SIM are that it is very fast, and it yields higher contrast when compared to wide-field microscopy. The drawback is that the reconstruction algorithm is complex and only a lateral resolution enhancement can be obtained. Another problem is that any imperfection in illumination quickly leads to image artifacts. In order to extend SIM to 3D, a three-beam variation of SIM was developed, which creates also a structured illumination along axial direction $[26,27]$. An axial structured illumination is generated by the interference of three coherent beams. 3D structured illumination results in super-resolution not only along the $x y$-directions, but also along the $z$-direction.

\subsubsection{Image Scanning Microscopy}

Image Scanning Microscopy (ISM) achieves higher resolution by scanning the sample with a single or multiple foci of excitation light, and then recording an image at each scan position. From this four/five-dimensional data (two/three dimensions of fcus position in the sample, plus two dimensions of the imaging camera), a super-resolved image can be computed, with a lateral resolution that twice better than that of a confocal microscope. This technique was first conceived by Colin Sheppard in 1988 and then experimentally realized by Müller and Enderlein in 2010 [18]. In this first implementation, the pinhole of the confocal microscope had been replaced by a camera, and an image was taken at every scan position of the focus. A super-resolved image can then be reconstructed by a very simple reconstruction algorithm which is called pixel reassignment [28]. There has been several successful applications of ISM in combination with other microscopy techniques [29-31].

As the laser scanning scheme mentioned above makes the image acquisition very time consuming, speeding up image acquisition is key for the application of ISM. This problem was firstly solved by a multi-focal ISM technique [32], which uses a DMD to realize a parallel confocal illumination with a sparse lattice of excitation foci. An image is then reconstructed by pixel reassignment and subsequent deconvolution. The reconstructed image achieves a resolution improvement of $\sqrt{2}$, and reaches a value of 1.63 
resolution enhancement after deconvolution.

Another way to speed up ISM is to combine it with Spinning Disk Confocal (SCD) Microscopy [33]. The laser scanning scheme mentioned above makes the image acquisition very time consuming. To overcome this limitation, a system combining ISM and Confocal Spinning Disk Microcopy (SDC-ISM) was developed, which is able to achieve about 1.5-fold resolution enhancement, while improving image contrast significantly. This technique is ideally suited for fast 3D super-resolution confocal imaging. A commercial version SDC-ISM system introduces an additional micro-lens array into the SDC module to realize an all-optical version of ISM, which makes ISM even faster [34].

The third version of ISM that I would like to mention here is rapid nonlinear ISM [35]. This technique combines ISM with two photon excitation microscopy, which can be used to excited fluorescence or for second-harmonic generation. This is also an alloptical version of ISM, based on the ideas of rescan ISM [36]. This method achieves high frame rates and high image contrast, which is very attractive for live cell imaging. Besides the above mentioned ISM variants, there exist many more variants of ISM, see Refs. [29, 37, 38].

\subsubsection{Deconvolution for Super-Resolution Microscopy Im- age Restoration}

Deconvolution is computational technique to improve image quality, which has been studied for a long time and has become a standard method for image quality improvement in fluorescence microscopy. During imaging, the object image is blurred by the PSF of imaging systems and will contain additional noise, such as detector thermal noise, readout noise, or sample background noise. Thus, the measured raw image is usually not optimal in resolution or contrast. However, if the PSF of the imaging system is known, and if one has a good model of the origin of the image noise, it is possible to restore an improved object's image by solving an inverse problem. Since imaging is equivalent to a convolution of the object (sample) function with the PSF, as just mentioned in section 1.2, and because this convolution is a linear operation, the deconvolution is computed as the inverse of this convolution. Although though there is an analytical solution for the deconvolution, the inverse of the convolution is an ill-posed problem due to the noise, which is the main problem of deconvolution in microscopy. A deconvolution with inappropriate parameters or inaccurate estimate of the PSF will not improve the resolution, but will generate artifacts in the de-convolved image. The achievable resolution enhancement depends on the signal-to-noise ratio (SNR) of the measured image, the accuracy of the PSF estimation, the noise model, and the used deconvolution algorithm.

Among existing deconvolution methods are linear deconvolution methods such as inverse filtering [39] and Wiener filtering [40], and non-linear deconvolution methods 
such as the Richardson-Lucy algorithm [41, 42], or regularized deconvolution [43]. There are many software packages available for deconvolution, such as the commercial software Huygens [44], or the open source software DeconvolutionLab2 [45].

\subsection{Scope of this thesis}

In this thesis, we are going to focus on linear super-resolution fluorescence microscopies and advanced deconvolution methods for super-resolution image restoration. In chapter 2, I present a deeper look onto SOFI and develop a deconvolution method to remove artifacts that are caused by the correlation calculation. In chapter 3, I describe the principle of SDC-ISM comprehensively, starting from the setup and ending with the final image reconstruction. In chapter 4, I present simulations of SIM. In chapter 5, I show how to solve L1-norm regularized minimization problems by non-linear optimization. In chapter 6, I study two types of Gaussian fitting algorithms for ISM confocal light-spot localization. In chapter 7, I develop a method for artifact removal in deconvolved super-resolution images. In chapter 8 , I study the image reconstruction problem for Airy light-sheet microscopy, and I summarize the whole work of my thesis in chapter 9, where I also give an outlook on possible future work. 


\section{Chapter 2 Super-resolution Optical Fluctuation Imaging}

\subsection{Introduction}

Existing super-resolution techniques, such as STED microscopy or PALM/STORM, are powerful but have also some drawbacks. STED requires very high excitation power for inducing stimulated emission (depletion), which can damage the sample and induce photo-bleaching; in contrast, PALM/STORM do not require high excitation intensities but are slow as they require the recording of many frames for reconstructing a single super-resolved image.

SOFI is a super-resolution technique that takes advantage of the temporal correlation of blinking emitters, and it achieves super-resolution by calculating the correlation of time traces in all pixels. It is quite simple to implement: a) prepare a sample by labeling it with blinking emitters and then record a sufficiently long movie of the labeled sample with a conventional wide-field microscope; b) calculate cumulants for each time trace pixel by pixel. In theory, an $n^{\text {th }}$ order cumulant image should result in a $\sqrt{n}$ times resolution enhancement. With deconvolution, this resolution enhancement can be increased to $\mathrm{n}$ times. Basically, a SOFI image is obtained by calculating the auto-correlation for a time trace separately all pixels. In practice, one calculates also often the cross-correlation between time traces of different pixels, to increase signal-to-noise ration and to eliminate finite-pixel-size artifacts. This process generates new virtual pixels and results in smoother images. The quality of a SOFI image can be further optimized by an adequate post-processing.

A first goal is to maximize resolution of SOFI. For $\mathrm{n}^{\text {th }}$ order SOFI, one increases the spatial frequency support by $\mathrm{n}$ times, but the SOFI image is still convolved with a $P S F=U^{n}(r)$, which gives only a gain of $\sqrt{\mathrm{n}}$ in optical resolution. In other words, SOFI still has the potential to gain more in resolution. Theoretically, this can be achieved either by going to higher order SOFI, or by improving the resolution with deconvolution. Several schemes have been proposed to maximize resolution by deconvolution, such as Richardson-Lucy deconvolution [41, 42].

A second goal is to linearize the SOFI image. An intensity non-linearization problem emerges when one goes to higher order SOFI. Higher order SOFI causes more serious non-linearization problems, because in $\mathrm{n}^{\text {th }}$ order SOFI, each emitter contributes to the final SOFI image proportionally to the $\mathrm{n}^{\text {th }}$ power of its actual brightness. This nonlinearity problem seriously hinders the application of high order SOFI. This is the reason why only $2^{\text {nd }}$ order SOFI is widely used in practice so far. Balanced SOFI tries to 
linearize intensity distribution of a SOFI image [46], but the results seems to improve the situation only slightly.

The last goal is artifact removal. The drawback of cross-correlation is that it causes very sharp checkerboard artifacts. In reference [20], a method has been proposed to smoothen the cross-correlation image, and it has been implemented by Theo Lasser's group at the EPFL [24]. However, it was found that it does not remove all artifact, especially for very noisy images.

In this chapter, I employ a simple but efficient approximate linearization method and introduce regularized deconvolution with a well-known regularization technique, the Tikhonov regularization, to remove artifacts in a SOFI image. Finally, the results will be compared to that of existing method.

\subsection{Theory of SOFI}

The principle of SOFI can be exemplified by imaging two emitters as shown in the following picture.

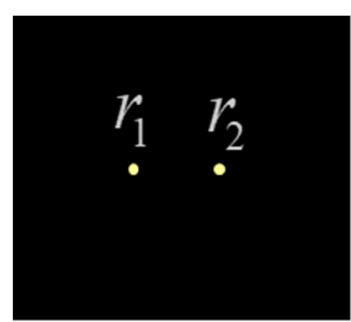

(a)

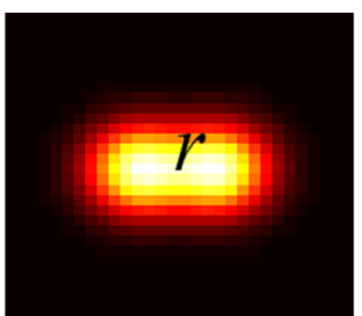

(b)

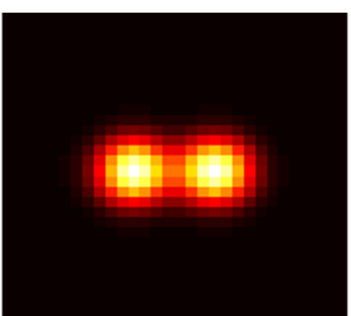

(c)

Figure 2.1 (a) Two emitters; (b) image taken by wide-filed microscope; (c) SOFI image.

Take $2^{\text {nd }}$ order SOFI as an example, the signal in a position $r$ generated by the two emitters can be represented as

$$
s(t)=s_{1}(t)+s_{2}(t)
$$

and the time-delayed signal (with lag time $\tau$ ) can be written as $s^{\prime}=s(t-\tau)$. Now we can determine the magnitude response with respect to position $r$ by autocorrelation as follows:

$$
\begin{aligned}
E\left(s s^{\prime}\right) & =E\left[\left(s_{1}+s_{2}\right)\left(s_{1}^{\prime}+s_{2}^{\prime}\right)\right] \\
& =E\left(s_{1} s_{1}^{\prime}\right)+E\left(s_{1} s_{2}^{\prime}\right)+E\left(s_{1}^{\prime} s_{2}\right)+E\left(s_{2} s_{2}^{\prime}\right)
\end{aligned}
$$

where $E$ means taking the mean value over time. Since the blinking between different emitters is statistically independent and it can be assumed a zero-mean stochastic process, the uncorrelated terms will be zero, so after calculation, we have

$$
C_{2}(r, \tau)=E\left(s_{1} s_{1}^{\prime}\right)+E\left(s_{2} s_{2}^{\prime}\right)
$$


which actually is equal to

$$
\begin{aligned}
C_{2}(r, \tau)=U^{2}(r- & \left.r_{1}\right) \cdot \varepsilon_{1}^{2} \cdot E\left[v_{1}(t) v_{1}(t-\tau)\right] \\
& +U^{2}\left(r-r_{2}\right) \cdot \varepsilon_{2}^{2} \cdot E\left[v_{2}(t) v_{2}(t-\tau)\right]
\end{aligned}
$$

where $U(r)$ is the PSF, $\varepsilon_{i}(i=1,2)$ is the brightness of an emitter, which can be regarded as constant, and $v_{i}(i=1,2)$ is the switch function of an emitter. In reality, there are usually only limited states that take place, e.g. for a quantum dot, so that $v$ will be a binary vector with elements $\frac{0}{1}$, which corresponds to discrete on/off states. So the pixels' value of a SOFI image is the sum of the product of the PSF, times brightness and autocorrelation of every single emitter. Due to the vanishing of the cross terms in the autocorrelation function, even very close emitters can be distinguished despite their overlap in the image. This is how SOFI can realize super-resolution. Although this is just a very simple case shown here, the theory can be expanded to cases with any number of emitters and for any order of SOFI. The $\mathrm{n}^{\text {th }}$ order SOFI can be written as

$$
C_{n}\left(r, \tau_{1}, \ldots, \tau_{n-1}\right)=\sum_{i=1}^{N} U^{n}\left(r_{i}-r\right) \cdot \varepsilon_{i}^{n} \cdot w_{i}\left(\tau_{1}, \ldots, \tau_{n-1}\right)
$$

where $U(r)$ is the PSF, $\varepsilon_{i}$ is the brightness of an emitter at location $r_{i}$, and $w_{i}\left(\tau_{1}, \ldots, \tau_{n-1}\right)$ is an autocorrelation based weighting function, which can be described by a cumulant [47]. Since it strongly depends on the fluctuation characteristics of each emitter, any un-correlated signal is suppressed. This endows SOFI with a very strong capacity to remove noise and background.

From the above derivation, for a $n^{\text {th }}$ order SOFI image, the PSF is replaced by the $n^{\text {th }}$ power of the original PSF. The shrinkage of the PSF is the key to the optical resolution enhancement. However, the non-linear effect of power-law scaling of the brightness of the emitters is a problem. Due to this effect, the intensity transform function from raw image to SOFI is actually scaling as $\varepsilon_{i}^{n}$, e.g. the cumulant magnifies the intensity by a power equal to the order number. For instance, an emitter that has a two times larger molecular brightness will appear $2^{\mathrm{n}}$ times brighter in a SOFI image of the $n^{\text {th }}$ order. Thus, cumulant calculation will result in a SOFI image which is eventually biased to larger intensities so that the contrast of a SOFI image is totally skewed as compared to the true sample brightness distribution. Furthermore, the computational complexity increases significantly with the increase of the order of SOFI. Non-linearity and high computational complexity are important problems at high orders. These problems require new and advanced image processing techniques to realize linear high-order SOFI.

\subsubsection{Cross-cumulant}

In the last section, I have shown that a SOFI image can be calculated by temporal correlation, in fact, it is also possible to calculate spatial correlation between pixels, because adjacent pixels contain information from emitters around [20]. For simplicity, I take the $2^{\text {nd }}$ order cross-cumulant as an example, which is defined by 


$$
\begin{array}{r}
X C_{2}\left(r_{1}, r_{2}, \tau_{1}, \tau_{2}\right)=\sum_{i=1}^{N} U\left(r_{i}-r_{1}\right) U\left(r_{i}-r_{2}\right) \varepsilon_{i}^{2} E\left[s_{i}\left(t+\tau_{1}\right) s_{i}\left(t+\tau_{2}\right)\right] \\
=U\left(\frac{r_{1}-r_{2}}{\sqrt{2}}\right) \sum_{i=1}^{N} U^{2}\left(r_{i}-\frac{r_{1}+r_{2}}{\sqrt{2}}\right) \varepsilon_{i}^{2} E\left[s_{i}\left(t+\tau_{1}\right) s_{i}\left(t+\tau_{2}\right)\right]
\end{array}
$$

The above formula shows that the cross-cumulant results in a "new pixel" at the geometric center of the cross-cumulated pixel-pair, and the intensity of the "new pixel" is modulated by a factor of $U\left(\frac{r_{1}-r_{2}}{\sqrt{2}}\right)$, which is correlated to the distance of the crosscumulated pixel-pair.

A feature of the cross-cumulant approach is that more pixels can be generated, while the weighting factor leads to intensity differences which results in sharp grid artifacts. In applications, these artifacts have to be removed to create a high-quality super-resolved SOFI image.

\subsection{Linearization and deconvolution}

In wide-field microscopy, imaging can be described as the convolution of the object function $F$ with the system's Point Spread Function (PSF), which results in a blurred image given by

$$
G=P S F * F
$$

Let us assume that the temporal correlation is identical for each emitter, then the widefield SOFI image can be written as

$$
\operatorname{SOFI}(n)=P S F^{n} *\left(W \cdot F^{n}\right)
$$

For deconvolution, the cost function can be written as

$$
E(F)=\left\|P S F^{n} *\left(W \cdot F^{n}\right)-\operatorname{SOFI}(n)\right\|^{2}
$$

Since cumulant calculations obey a power law, it is easy to see that taking the $\mathrm{n}^{\text {th }}$ root of a SOFI image linearizes the brightness response:

$$
G=\sqrt[n]{S O F I}
$$

This is the simplest way to linearize a SOFI image approximately, which does not involve complex calculation. Theoretically, the final super-solved image can be restored via deconvolution, which maximizes the resolution of a SOFI image. Although the Fourier domain support of a SOFI image has been doubled as compared to that of a widefield microscopy image, the PSF is not optimal. Therefore, a linear super-resolution image needs to be further enhanced by linear deconvolution. That is why I employ here a linear robust deconvolution technique to retrieve the object. Unlike a RichardsonLucy algorithm, which can be used for efficient deconvolution, it is easy to generate artifacts without regularization. Besides, it is actually a non-linear deconvolution technique; a non-linear deconvolution is not appropriate for the linearization of SOFI image. 
Based on the deconvolution model mentioned above, it is easy to write the objective function with Tikhonov regularization as follow

$$
E(f)=\frac{1}{2}\|H * f-g\|_{2}^{2}+\lambda\|f\|_{2}^{2}
$$

where $f$ is a vector of one-dimensional $F, \lambda$ is a regularization parameter, $\|\cdot\|_{2}$ mean 2-norm, $g$ is vector of $G$, and $H$ is the convolution matrix define by the PSF which causes blurring. This is a classic linear inverse problem which can be solved by a Conjugate Gradient algorithm.

For calculation purposes, (2.11) can be to be transformed into a non-constraint optimization problem. We set $f=x^{2}$ and $X$ a diagonal matrix with $X_{i i}=x_{i}$ [48], then the objective function is modified to

$$
E(x)=\frac{1}{2}\left\|H * x^{2}-g\right\|_{2}^{2}+\lambda\left\|x^{2}\right\|_{2}^{2}
$$

and the gradient can be written as

$$
\nabla E=2 X\left[H^{T}\left(H x^{2}-g\right)+2 \lambda x^{2}\right]
$$

where symbol $T$ means transposition. This explicitly induces a non-negative constraint. With a given PSF, the object function can be retrieved by minimizing (2.12) using any un-constraint optimization method.

\subsection{Results}

In this section, the proposed algorithm is checked by simulation and experimental data analysis, and the results are then compared to existing methods.

\subsubsection{Simulation}

The simulation is based on the software tool described in Ref. [49]. The ground-truth of the object is shown in Figure 2.2. The positions of the emitters are generated randomly, and each emitter is represented by a Gaussian distribution.

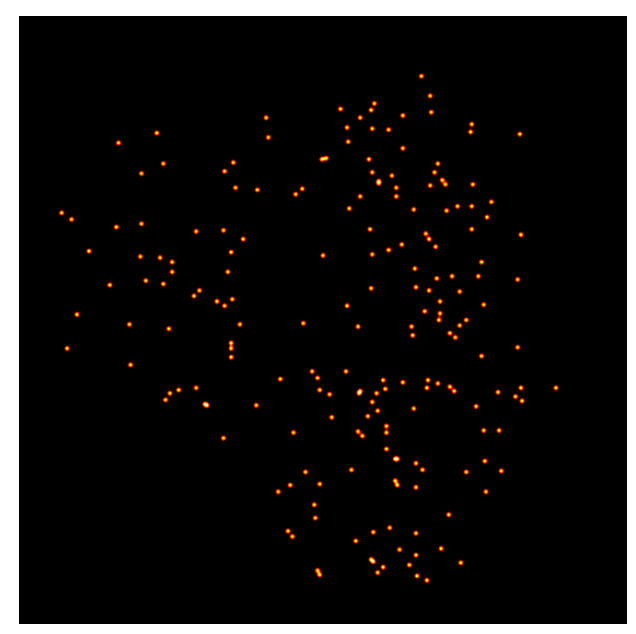

Figure 2.2 
The De-convolved SOFI images generated by two methods are shown in Fig. 2.3, where the first row shows the $2^{\text {nd }}$ order SOFI image and the corresponding de-convolved image, the middle row shows the $3^{\text {rd }}$ order SOFI image and the corresponding de-convolved image, and the third row shows the $4^{\text {th }}$ order SOFI image and the corresponding de-convolved image.
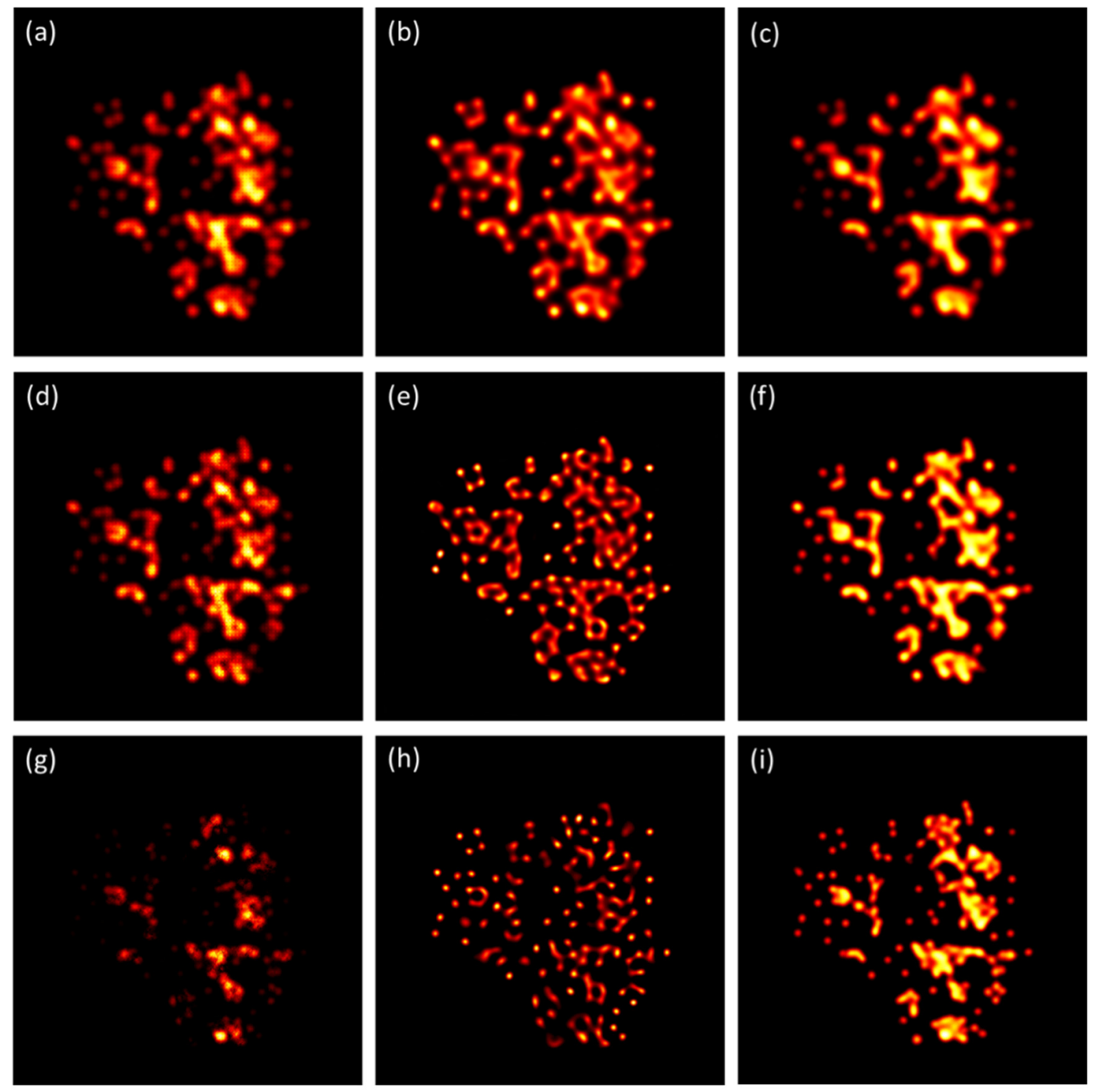

Figure 2.3 Left column: 2 4-order SOFI image. Middle column: results by proposed method. Right column: results by existing method

The simulation results show that the proposed method outperforms the existing method, especially for high order SOFI image deconvolution. The existing method has a good performance at low label density. However, its performance becomes very poor at high label density.

\subsubsection{Experiment}

I tested my algorithm using experimental SOFI data, in which a raw stack of images 
contains 3000 recorded frames of a sample, Rat hippocampal neurons with immunestained neurotransmitter receptor subunit GABABR1, with labels of quantum dots QD525 (Invitrogen). For the sake of computing time, I performed the analysis on a truncated $(128 \times 128)$ sub-image of the full image.

In order to guarantee a smooth cumulant result, I calculated $2^{\text {nd }}$ and $4^{\text {th }}$ order cumulants via Fourier SOFI [50], which employs Fourier interpolation to increase the grid density of a SOFI image. To linearize the SOFI image, I took the $\mathrm{n}^{\text {th }}$ root of the $\mathrm{n}^{\text {th }}$ order SOFI image. The PSF is estimated from the raw image by fitting a Gaussian function to the image of a single quantum dot, and the resulting FWHM of this function is 1.1190 pixels. Finally, I de-convolved the linearized SOFI image by a nonlinear Conjugate Gradient algorithm to solve eq. (9). The results are shown in Figs. 2.4 and 2.5.
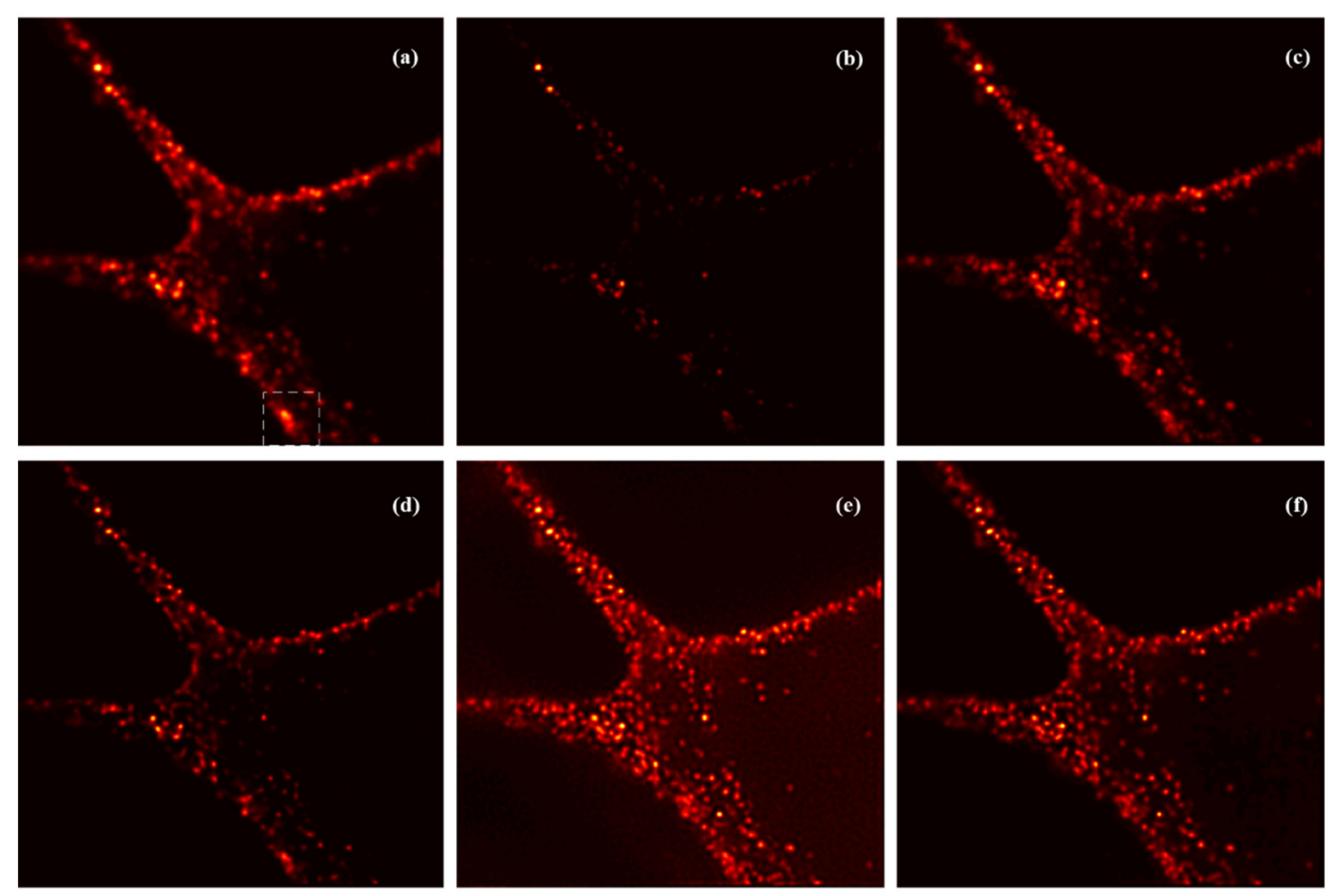

Figure 2.4. Image processing results for experimental images: (a) $2^{\text {nd }}$ order SOFI image; (b) $4^{\text {th }}$ order SOFI image; (c) square root of (b); (d) deconvolution to (a) directly by RL algorithm; (e-f) deconvolution to linearized (b) by RL algorithm (f) and proposed method.

Fig. (c) shows the deconvolved $2^{\text {nd }}$-order SOFI image shown in Fig.(a). Even though the deconvolved $2^{\text {nd }}$-order SOFI image is improved significantly, its resolution is still not parallel to higher order SOFI image, which can be told by naked eye.

Fig. 2.4 (c) shows the square root of a $4^{\text {th }}$ order SOFI image. We can see that this higher order SOFI image shows more details than the $2^{\text {nd }}$ order SOFI image. For better comparison, Fig. 2.5 shows the results side by side; an area with high density labels is selected for resolution comparison. 

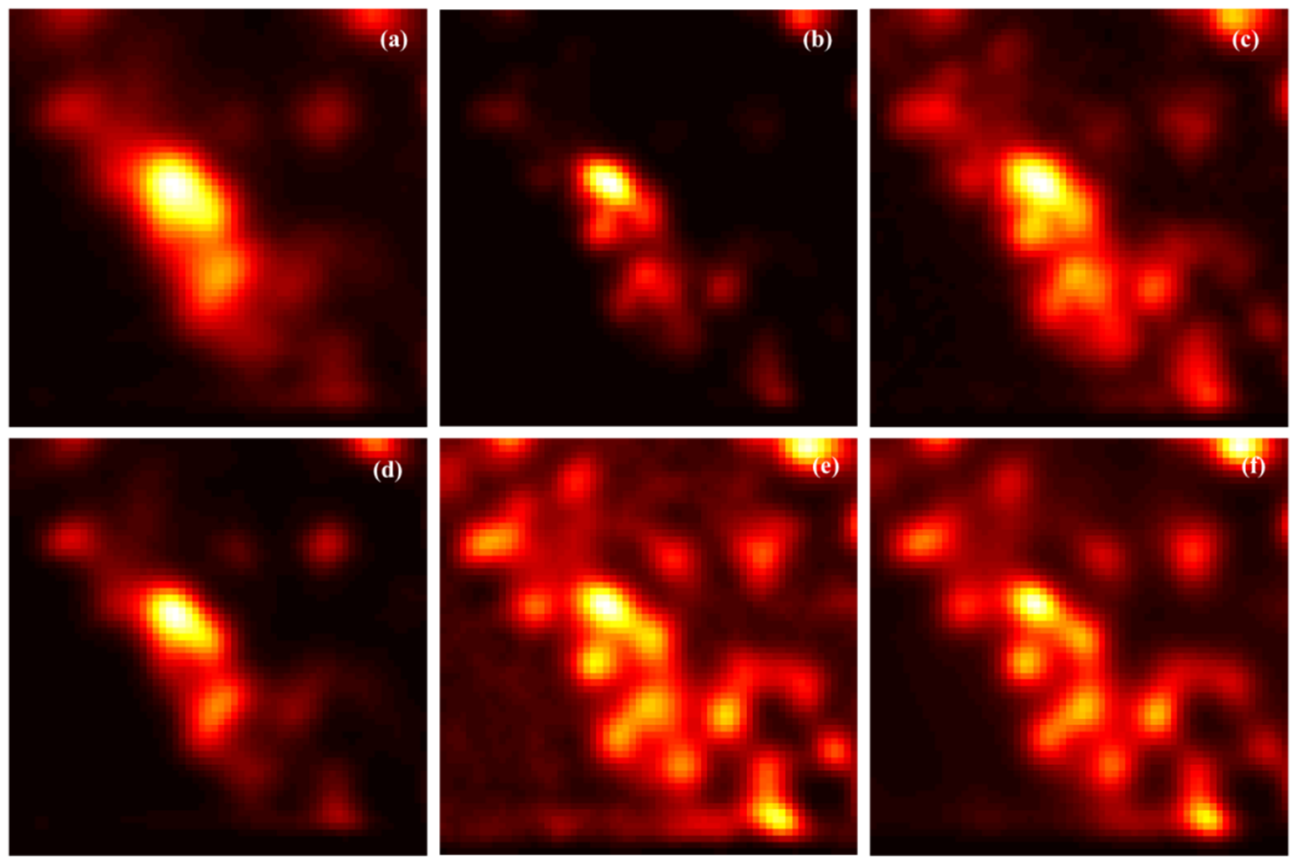

Figure 2.5 Zoom-in images in area inside the rectangular marked in figure 2.4 (a) of each figure in

figure 2.4

In this figure, we can see that the de-convolved $4^{\text {th }}$ order SOFI images show a higher resolution than the $2^{\text {nd }}$ order SOFI image. Roughly, resolution is increased by about two times. In addition, the contrast of de-convolved $4^{\text {th }}$ order SOFI image looks better than that of the $2^{\text {nd }}$ order SOFI image. By deconvolution with the proposed method, the image resolution and image quality are improved significantly. In contrast, even though Richardson-Lucy algorithm also results in high resolution, it causes a lot of artifacts.

\subsection{Conclusions}

In this chapter, I presented a simple but efficient approach for how to realize a linearized high order SOFI deconvolution. I analyzed the theory of how to improve the optical resolution by higher SOFI, and I solved the problem of resolution maximization and intensity linearization for high order SOFI. I showed that higher resolution and high quality of high order SOFI images can be obtained by deconvolution with regularization; SOFI image can be linearized approximately just by taking its $\mathrm{n}^{\text {th }}$ root; with deconvolution, the optical resolution can be improved significantly. I validated my method on experimentally measured $4^{\text {th }}$ order SOFI images of a sample that was labeled with quantum dots. The results show that higher order SOFI image give true optical resolution enhancement; deconvolution with regularization has more advantages than existing methods in artifact removal. 


\section{Chapter 3 Spinning Disk Confocal - Image Scanning Microscopy}

\subsection{Introduction}

Spinning disk confocal (SDC) microscopy solves the problem of low image acquisition speed of conventional laser scanning microscopy by introducing a spinning disk, which allows scanning with thousands of beams in parallel. In this way, the speed of confocal scanning can be increased significantly. However, the lateral resolution is still the same as before and equal to that of a wide-field microscope, although confocal microscopy is capable of $z$-sectioning. Image Scanning Microscopy (ISM) is a clever technique for super-resolution confocal microscopy, which has been demonstrated to be compatible also with SDC microscopy.

A typical setup of a SDC microscope consists of a standard microscope containing an objective, a tube lens, a sample stage, and the confocal spinning disk unit (CSU) [51]. This unit outputs a synchronization signal indicating the position of the spinning disk, connected to a camera port of the microscope. ISM is very easy to implement based on an existing SDC system. Technologically, one just needs to synchronize the camera, excitation laser, and the CSU to measure the confocal light spot image at each scan position. The image is then reconstructed by resampling the area around each illumination spot to improve the resolution by a factor of $\sqrt{2}$, and then summing up all the upsampled images. Finally, an ISM image achieves a two-fold resolution enhancement after de-convolution.

The SDC-ISM system has been built and applied by Olaf Schulz at al. [33]in 2013 . The details of resolution analysis have been presented there, together with several biological applications. It is very worth to spread this novel technique to broader audiences, since there exist so many SDC microscopes all over the world. Therefore, I have developed a precise and flexible control unit and an easy-to-use software for an upgrade of an SDC system to SDC-ISM. The developed software will be packaged and published online, so that any potential user can upgrade their existing SDC system to SDC-ISM setup quickly.

In this chapter, I show the theory of ISM and explain why ISM have the capacity of super-resolution and how to maximize the resolution of ISM image. I also show the design of the FPGA core for synchronizing signal generation and the design of image acquisition software based on the open source platform MicroManager Plugin. Finally, I introduce the method for reconstruction of ISM image. 


\subsection{Theory of Image Scanning Microscopy}

To understand the principle of ISM, confocal light spot reassignment is the key. Imagine that a sample is canned with a focal spot. For the mathematical analysis, we denote the sample space coordinate by $r^{\prime}$, the CCD space coordinate by $s$, and with $r$ the scan focus position. Furthermore, $P(r)$ is the excitation PSF, and $U(r)$ is the emission PSF. We assume that both PSFs are perfectly symmetric, so that we have

and

$$
P(r)=P(-r)
$$

$$
U(r)=U(-r)
$$

For wide-field microscopy ISM, in coordinate of 2D image sensor (e.g. the camera), the respond of laser excitation at position $r$ can be represented as

$$
I(r, s)=\int U\left(s-r^{\prime}+r\right) P\left(r-r^{\prime}\right) f\left(r^{\prime}\right) d r^{\prime}
$$

which forms the confocal light sports image of ISM imaging.

Based on the measured image by (3.3), there are two ways to reconstruct an image basically. The most simple way would be integrating $I(r, s)$ over $\mathrm{s}$,

$$
I(r)=\iint U\left(s-r^{\prime}+r\right) d s P\left(r-r^{\prime}\right) f\left(r^{\prime}\right) d r^{\prime}
$$

which lead to

$$
I(r) \propto \int P\left(r-r^{\prime}\right) f\left(r^{\prime}\right) d r^{\prime}
$$

This is indeed the process of general conventional confocal microscopy with a large pinhole. For general confocal microscopy, the resolution is mainly determined by excitation PSF; with a large pinhole, the resolution is close to wide-field microscopy. The other way would be integrating $I(r-s, s)$ over $\mathrm{s}$,

$$
I(r)=\iint P\left(r-s-r^{\prime}\right) d s U\left(-r^{\prime}+r\right) f\left(r^{\prime}\right) d r^{\prime}
$$

which lead to

$$
I(r) \propto \int U\left(r-r^{\prime}\right) f\left(r^{\prime}\right) d r^{\prime}
$$

The effective PSF become $U\left(r-r^{\prime}\right)$, which means that this is actually an image measured by the wide-field microscopy. Both image reconstruction methods result in diffraction limited resolution.

In fact, the optimal reconstruction method should be integrating $I\left(r-\frac{s}{2}, s\right)$ over s, 


$$
I_{I S M}(r)=\iint P\left(r-r^{\prime}+\frac{s}{2}\right) U\left(r-r^{\prime}-\frac{s}{2}\right) d s f\left(r^{\prime}\right) d r^{\prime}
$$

which lead to an effective PSF of ISM image by

$$
P S F(r)=\int P\left(r+\frac{s}{2}\right) U\left(r-\frac{s}{2}\right) d s
$$

It would be convenient to study the performance of ISM in Fourier domain. The Fourier transformation of (3.9) can be derived and finally the OTF of wide-field ISM is given by

$$
\operatorname{OTF}(q, w)=2 \pi \int U\left(\frac{q}{2}, w-w^{\prime}\right) P\left(\frac{q}{2}, w^{\prime}\right) d w^{\prime}
$$

It shows that the OTF of ISM is obtained by stretching the general wide-field OTF along lateral direction by factor of 2 , and then convolve by itself by z-direction.

(A)

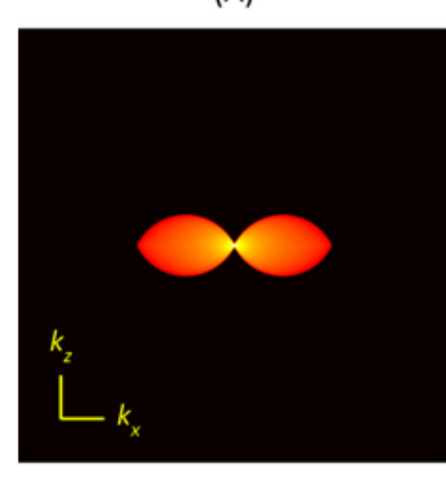

(B)

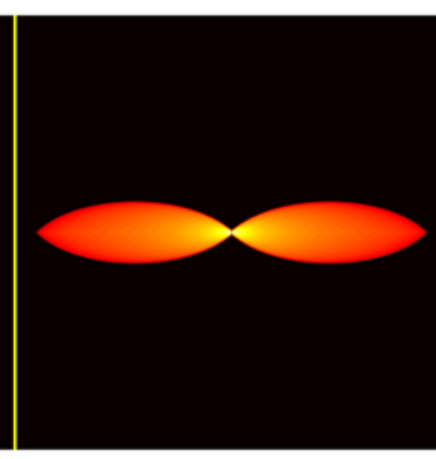

(C)

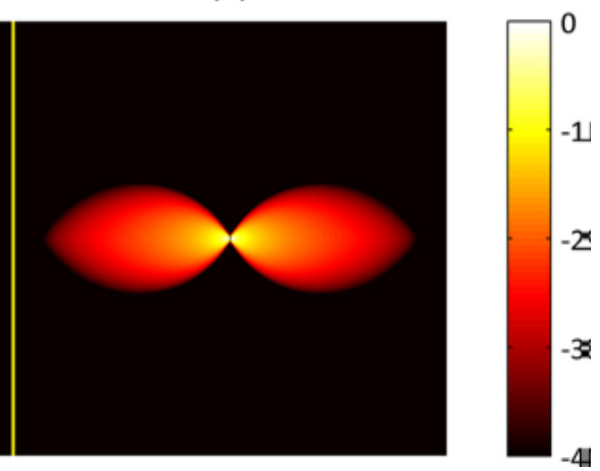

Figure 3.1 (A) OTF of wide-field microscopy. (B) Laterally stretched OTF of wide-field microscopy. (C) OTF of wide-field ISM. Images taken from Ref. [9].

Fig 3.1 shows that the shape of OTF of wide-field ISM is quite similar to that of a widefield microscopy. There is almost no resolution enhancement in z-direction. The above analysis shows that he resolution enhancement is mainly dependent on both of excitation PSF and emission PSF. The performance of the ISM resolution enhancement can be estimated by

$$
\frac{1}{\sigma_{i s m}^{2}} \approx \frac{1}{\sigma_{e x}^{2}}+\frac{1}{\sigma_{d e}^{2}}
$$

which shows that only when both of PSF are identical, can ISM achieve the highest resolution enhancement. Otherwise, the resolution will be mainly determined by the PSF with minimal FWHM, take STED for example, ISM will not improve resolution to STED, since the excitation PSF of STED is much sharper than its detection PSF.

In case of confocal microscopy, the measured ISM image can be represented by

$$
I(r, s)=\int A(s) U\left(s-r^{\prime}+r\right) P\left(r-r^{\prime}\right) S\left(r^{\prime}\right) d r^{\prime}
$$

where $\mathrm{A}$ is pinhole function. Apply ISM as above analysis to wide-field ISM, we have 


$$
\begin{aligned}
I_{I S M}(r) & =\int I\left(r-\frac{s}{2}, s\right) d s \\
& =\iint A(s) U\left(r-r^{\prime}+\frac{s}{2}\right) P\left(r-r^{\prime}-\frac{s}{2}\right) S\left(r^{\prime}\right) d s d r^{\prime}
\end{aligned}
$$

The PSF of ISM image is given by

$$
P S F_{i s m}=\int A(s) U\left(r+\frac{s}{2}\right) P\left(r-\frac{s}{2}\right) d s
$$

The derivation of OTF of confocal ISM can be found in reference [33]. Fig 3.2 shows the OTF of confocal ISM versus OTF of wide-field microscopy and confocal microscopy. Similar to wide-field ISM, the final resolution enhancement of confocal ISM only takes place in lateral direction and the resolution along optical axis keep the same as confocal microscopy.

(A)

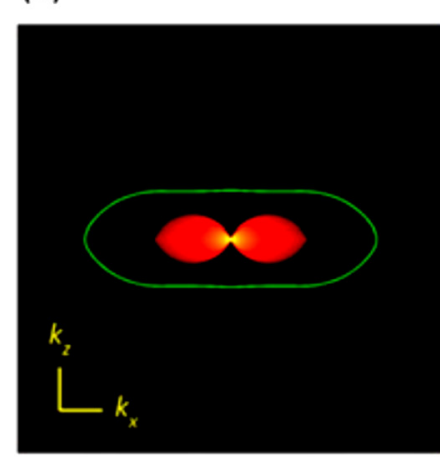

(B)

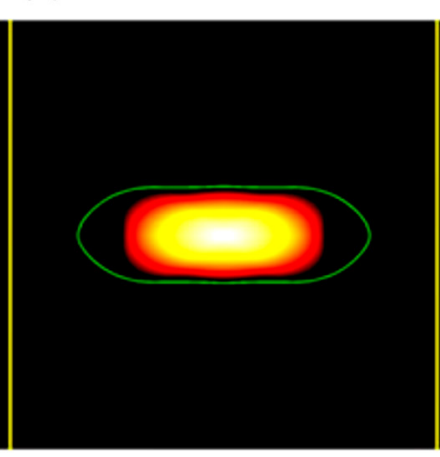

(C)

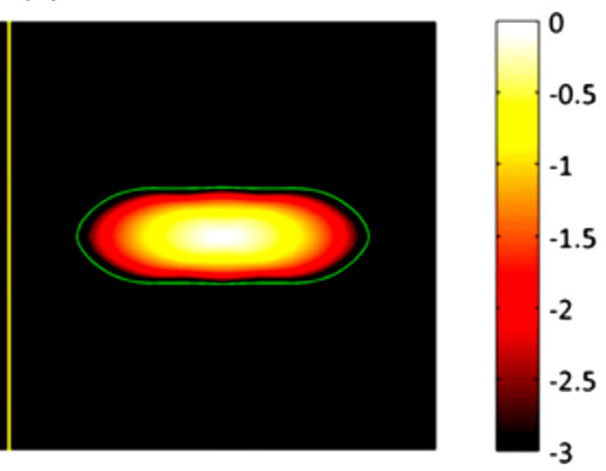

Figure 3.2 (A) OTF of wide-field microscopy. (B) OTF of confocal microscopy. (C) OTF of confocal ISM [33].

Based on the above analysis, one will find that the OTF of ISM is actually approximately equal to the multiplication of the laterally-stretched excitation OTF and detection OTF, which results in that the intensity the amplitude distribution of the OTF of ISM is not optimal as that of a general microscope objective. Therefore, the reconstructed ISM image needs be modified by Fourier reweighting, which can be realized by applying the following filter

$$
W(k)=\frac{1}{U\left(\frac{k}{2}\right)+\varepsilon}
$$

where $U\left(\frac{k}{2}\right) \approx P\left(\frac{k}{2}\right)$ and $\varepsilon$ is a parameter that avoid noise amplification in high frequencies in Fourier domain, to the reconstructed ISM image.

The above ISM imaging model analysis shows that ISM is indeed a very clever image measurement and reconstruction method to obtain linear super-resolution. As all pixels 
of measured images are used for reconstructing an ISM image, no photon is lost, therefore, the image contrast can be improved significantly. Besides, it is very easy way to be realized on a computer, and the computation cost is very small.

\subsection{SDC-ISM System}

In this section, I discuss the principle of confocal ISM with a spinning disk unit designed by Yokogawa ${ }^{9-}$, (e.g. CSU-X1) which is among the most advanced and popular commercial systems. Compared to other super-resolution methods, ISM is a method which can be realized in a straightforward manner for achieving super-resolution imaging in SDC microscopy. The combination of SDC and ISM (SDC-ISM) results in a fast super-resolution confocal microscopy. The concept of SDC-IMS can be described based on Fig. 3.3.

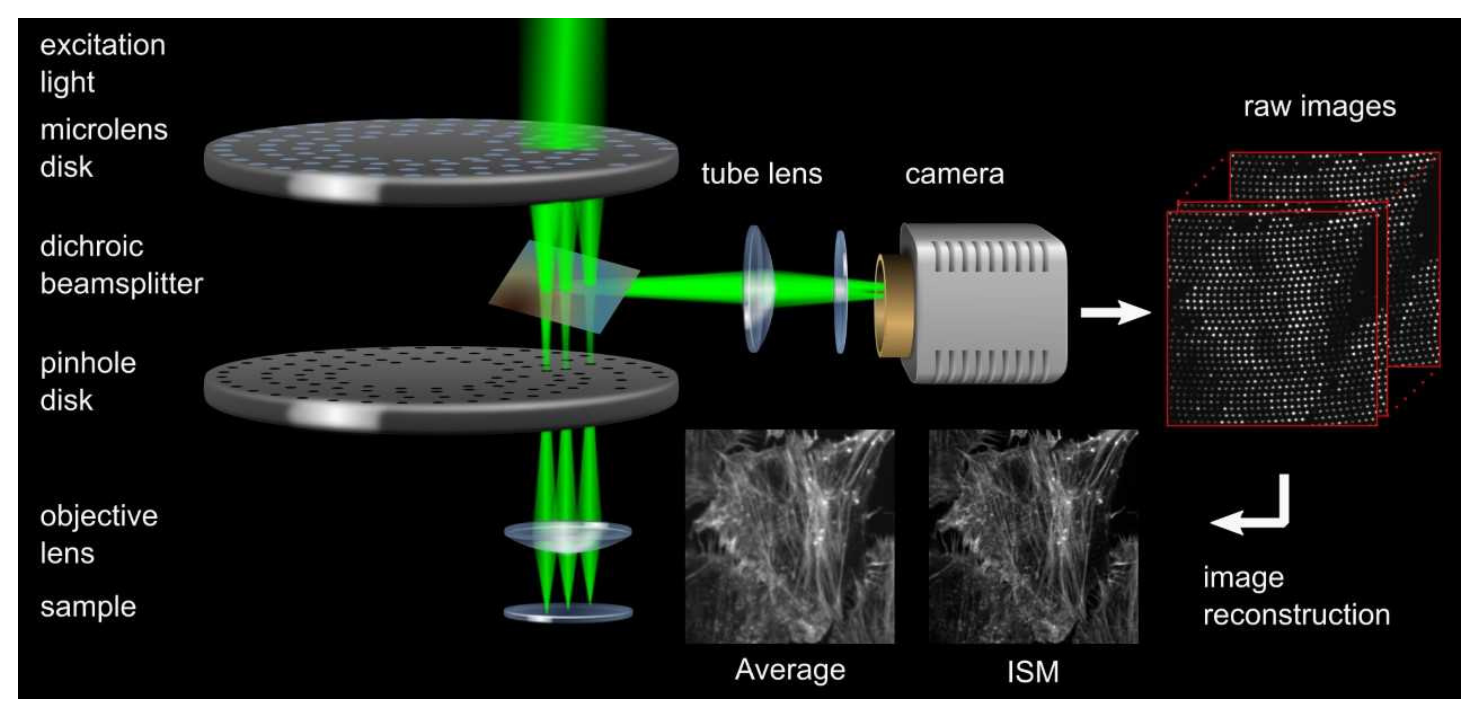

Figure 3.3 Schematic diagram of SDC-ISM. Image taken from Ref. [52].

Instead of scanning continuously, SDC-ISM scans the sample for a series of exact rotation angles of the spinning disk. Each frame corresponds to one rotation angle, and the fluorescence light that can go through the pinholes forms an ISM raw image. Because the measured confocal light spots do not overlap in these images, a fairly simple reconstruction algorithm can be derived to obtain a super-resolved image.

The hardware is shown in Fig. 3.4. Basically, the hardware consist of a commercial wide-field microscope, a spinning disk unit, a camera, a multi-wavelength laser system, a computer with a plugin FPGA, and a cable hub. All these modules are quite common and available in many bio-imaging labs, except the FPGA. In fact, the FPGA board is also a very common device nowadays, there are many function-integrated products available, take NI FPGA board for example. Therefore, the hardware of SDC-ISM is quite accessible and easy to build based on an existing setup. In our project, NI FPGA board is selected for its friend interface to the application on desktop computer, by which commend or data can be send to and received from FPGA by programming. 


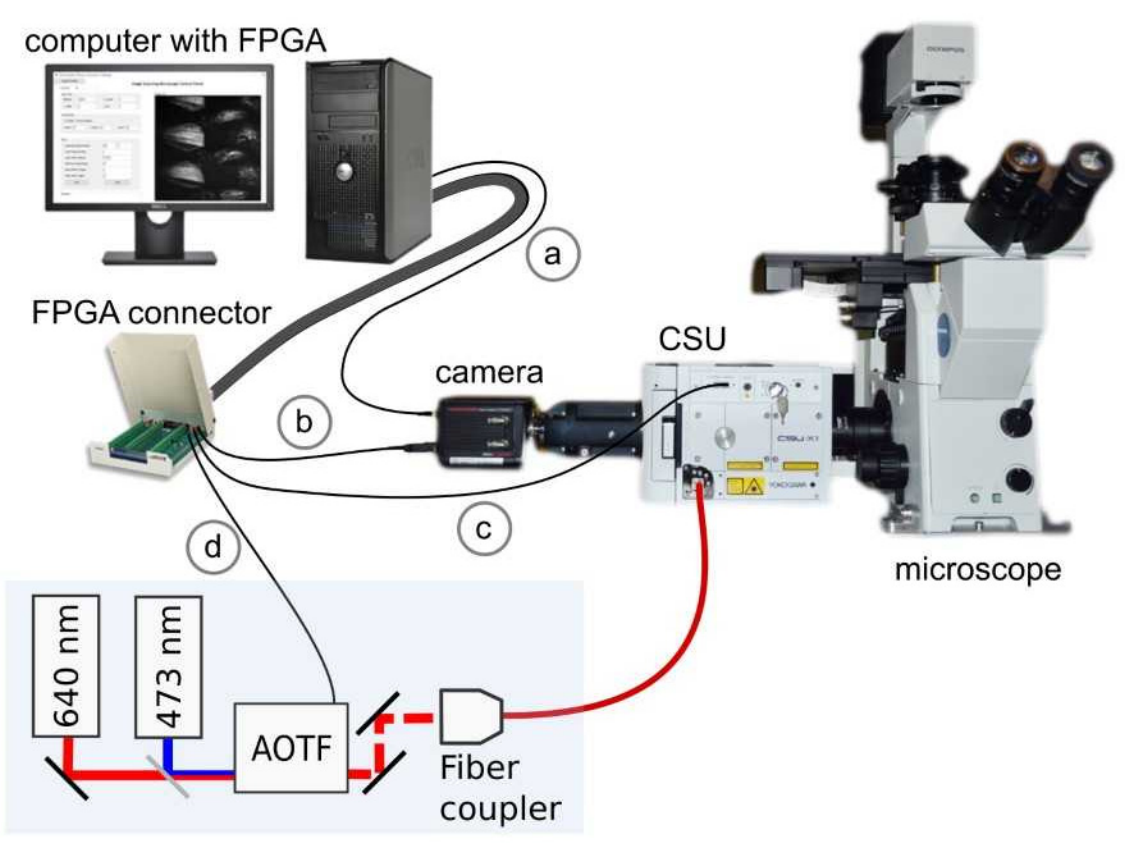

Figure 3.4 SDC-ISM hardware system [52].

The whole system is based on an existing SDC system. With an AOTF, lasers of different color are coupled to the spinning disk unit via an optical fiber. The FPGA board is embedded in a computer via a PCI interface. The input/output pins of the FPGA are extended by a cable and a NI terminal box. Thus, input signal comes from the SDC unit (cable $\mathrm{c}$ ), the control signals are transmitted by cable from the terminal box to the camera (cable b) and laser (cable d), and the acquired data of the camera is transferred via a special cable (cable a) to the computer.

In the CSU, the input light is collimated to a large perfectly shaped beam and projected onto a rotating disk. In this disk, there are many individual micro-lenses arranged in a dedicated pattern of nested spirals. The micro-lenses focus the excitation light that passes a dichroic mirror and reaches a second disk containing small pinholes at the positions corresponding to the foci of the micro-lenses in the first disk. After going through the pinholes, the foci are projected into the sample. In this way, a multitude of parallel and well-separated scanning beams excite the sample. Fluorescence is collected in epi-configuration, projected back through the pinholes and reflected by the dichroic mirror between the disks. Finally, a lens projects the fluorescent light to a camera. The synchronous rotation of the two disks realizes a multi-laser-beam scanning of the sample. After rotation of the disks by a certain angle, the beams have scanned the sample evenly and the image is read out from the camera. Thus, SDC microscopy accelerates imaging by scanning the sample with many confocal laser beams in parallel.

Based on the hardware scheme shown in Fig. 3.2, I present a software package to upgrade any commercial spinning disk microscope with this fast super-resolution option without compromising the function of the microscope in any way. In particular, this upgrade does not require to change any optical components in the spinning disk unit. 


\subsection{Image Reconstruction}

An ISM image is constructed from a stack of images each recorded at a different scan position. Each image consists of individual spots determined by the pinhole positions of the disk (see Fig 5). If the positions of the pinholes are known, the final ISM image is obtained by pasting the spots to a new pixel grid that is twice as large. This is repeated for every image of the stack.

a

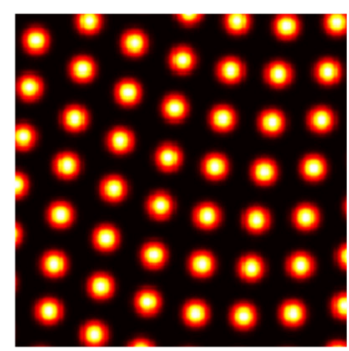

b

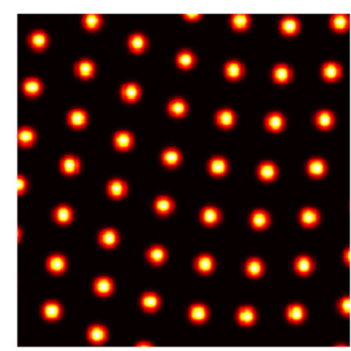

Figure 3.5 Principle of ISM reconstruction. (a) One raw image $(\mathbf{N} \times \mathbf{N})(\mathrm{b})$ Reconstructed ISM image $(\mathbf{2} \mathbf{N} \times \mathbf{2 N})$. During reconstruction, the relative distances between spots are always kept the same. Technically, this can be easily implemented by copying each spot from the raw image with a proper window and paste it to the same relative position in the new pixel array. Actually, the shape of a single spot in the ISM image does not change, but the size of an ISM image is doubled, so the light spots look sharper compared to the raw image.

The reconstruction algorithm for an ISM image can be described by the following steps:

Step1: Record a stack of reference images from a sample with a homogeneous intensity distribution (e.g. a dye solution).

Step2: Localize the precise positions of each pinhole $\left(\mathrm{x}_{\mathrm{I}}, \mathrm{y}_{\mathrm{I}}\right)$ in each reference image.

Step3: Create an empty grid $R$ of size $(2 \mathrm{~N} \times 2 \mathrm{~N})$ if $\mathrm{N}$ is the size of the camera image.

Step4: For each sample image, copy the pixels covering the spot around $\left(\mathrm{x}_{\mathrm{I}}, \mathrm{y}_{\mathrm{I}}\right)$ and add them at the position $\left(2 \mathrm{x}_{\mathrm{I}}, 2 \mathrm{y}_{\mathrm{I}}\right)$ to $\mathrm{R}$.

In ISM image reconstruction, it is necessary to take a reference measurement to calculate the position at every scan position. This, however, just needs to be done once, and only steps 3 and 4 are needed to calculate an ISM image, so the computational cost is relatively small. The algorithm is exceptionally easy to implement and, for example, much simpler than the mathematics needed for computing a SIM image.

\subsection{Synchronization and Laser pulsing control}

The key to SCD-ISM is to obtain images of all light spots of the confocal pinholes by a camera at precise positions of the disks. Since the disks are continuously spinning, 
one must avoid motion artifacts. This is achieved by a stroboscopic illumination. The lasers are on just short enough ( $\sim 6 \mu \mathrm{s})$, so that the disk can be considered to be stationary. Furthermore, the time to switch on the laser must be highly accurate, such that the pinhole position is strictly the same as for the reference image. Therefore, the triggering of the lasers and the camera requires a precise timing control that must be also synchronized to the spinning disk rotation. A Field Programmable Gate Array (FPGA) is the method of choice to realize this. The online synchronization during the measurement of the spinning disk position and the laser pulses provide a high reproducibility of the positions of the pinholes on the camera. The FPGA is controlled by our Micro-Manager $(\mu \mathrm{M})$ plugin that runs an embedded program on freely available FPGA hardware.

Lasers and camera have to be synchronized to the CSU. In each scanning cycle, $N_{\mathrm{P}}$ laser pulses are fired with a uniform spacing. In practice, more scanning cycles can be used to improve the signal as one achieves a longer exposure time and more fluorescence light is captured by the camera. For the next frame of the total stack of ISM images, a delay is added to the timing of the laser pulses. This effectively shifts the spots to the next scan position. In practice, the selection of the number of frames $\left(N_{\text {ISM }}\right)$ and the number of laser pulses $\left(N_{\mathrm{P}}\right)$ are important parameters for SCD-ISM imaging. Choosing a too small value for $N_{\text {ISM }}$ leads to potential under-sampling and artifacts can occur during reconstruction. Optimally, half-overlap of the spots between two neighboring frames is desirable. On the other hand, more frames reduce the total speed of acquisition. By increasing $N_{\mathrm{P}}$, more scan positions are imaged in one frame. To maximize efficiency, $N_{\mathrm{P}}$ should be as large as possible. However, one must avoid overlapping of light spots within a frame. Experimentally, we find that the optimal number of laser pulses is 4 or 6 with 250 frames.

\subsubsection{Analysis of Laser Triggering}

Basically, the timing control is based on a precise clock-driving counter. In the FPGA, a counter $\mathrm{c}$ is used as a ticker, which is driven by a clock and has a precision of $25 \mathrm{~ns}$, e.g. the clock period, and it is used to measure the single image-scanning period. $C$ is clear when the rising edge of the spinning-disk signal arrives and starts to count and measure the next cycle. Another register $c_{0}$ is used to store the measured value by the counter $c$. One cycle of the spinning-disk signal can be divided into many time slots so that the relationship between counting, pulsing and imaging can be recovered. Firstly, it is divided into $N_{p}$ sections, each section takes place of one pulse and assumes that the gaps between pulses are uniform; then each section is divided into $N_{i}$ units. The time slot can then be determined by

$$
\Delta T=\frac{c_{0}}{N_{i} N_{p}}
$$

where $N_{i}$ and $N_{p}$ are the number of frames to be taken and the number of laser pulses for imaging each frame, respectively. Then delay the pulses with an increment of $\Delta T$, and after each delay, a single frame is taken; finally, $N_{i}$ images are measured by $N_{i}$ steps of $N_{p}$-pulses shifts. Observe by an oscilloscope how the laser triggering pulses 
shift from left to right and go through a single cycle of the spinning-disk signal. To realize a complete scan, the pulse-shifting should be done as a circle-shifting and it should stop one step, e.g. by time $\Delta T$, before the pulses of the last frame overlap the pulses of the first frame again. According to this condition, the rising moment of each laser pulse in each cycle of the spinning-disk signal can be determined by

$$
t_{n}=\Delta T \cdot\left(n \cdot N_{i}\right)+\Delta T \cdot m
$$

Where $n=\left[0,1, \ldots, N_{p}-1\right]$ is the index of the laser pulse in each cycle, and $m=$ $\left[0,1, \ldots, N_{i}-1\right]$ is the index of the frame. For simplicity, let the laser pulse width $\mathrm{W}_{\mathrm{p}}$ be equal to $2 \Delta T$, namely $W_{p}=2 \Delta T$, then a laser pulse can be generated by calculating in the FPGA

$$
t_{n} \leq c \leq t_{n}+2 \Delta T
$$

which, after substituting into (3.17), leads to

$$
\left\{\begin{array}{l}
c \geq \Delta T \cdot\left(n \cdot N_{i}\right)+m \cdot \Delta T \\
c<\Delta T \cdot\left(n \cdot N_{i}\right)+m \cdot \Delta T+2 \Delta T
\end{array}\right.
$$

Substituting $\Delta T$ by $\frac{c_{0}}{N_{i} N_{p}}$, we have

which finally yields

$$
\left\{\begin{array}{l}
c \geq \frac{c_{0}}{N_{i} N_{p}} \cdot\left(n \cdot N_{i}\right)+m \cdot \frac{c_{0}}{N_{i} N_{p}} \\
c<\frac{c_{0}}{N_{i} N_{p}} \cdot\left(n \cdot N_{i}\right)+m \cdot \frac{c_{0}}{N_{i} N_{p}}+2 \frac{c_{0}}{N_{i} N_{p}}
\end{array}\right.
$$

$$
\left\{\begin{array}{l}
c \cdot N_{i} N_{p} \geq c_{0} \cdot\left(n \cdot N_{i}\right)+c_{0} \cdot m \\
c \cdot N_{i} N_{p}<c_{0} \cdot\left(n \cdot N_{i}\right)+c_{0} \cdot m+2 c_{0}
\end{array}\right.
$$

The above transformations significantly simplify the calculation of the pulsing moments. Technically, the above inequality can be easily implemented on the FPGA by using only multiplication, addition and comparison operations.

More generally, let the width of a laser pulse be variable. In that case, the time of risingedge and the time of falling-edge of the laser pulses can be determined in the following way:

Condition I: $t_{n} \leq c<t_{n}+W_{p}$

$$
\left\{\begin{array}{l}
c \geq \Delta T \cdot\left(n \cdot N_{i}\right)+m \cdot \Delta T \\
c<\Delta T \cdot\left(n \cdot N_{i}\right)+m \cdot \Delta T+W_{p}
\end{array}\right.
$$

substitute $\Delta T$ by $\frac{c_{0}}{N_{i} N_{p}}$, we have 
which is equal to

$$
\left\{\begin{array}{l}
c \geq \frac{c_{0}}{N_{i} N_{p}} \cdot\left(n \cdot N_{i}\right)+m \cdot \frac{c_{0}}{N_{i} N_{p}} \\
c<\frac{c_{0}}{N_{i} N_{p}} \cdot\left(n \cdot N_{i}\right)+m \cdot \frac{c_{0}}{N_{i} N_{p}}+W_{p}
\end{array}\right.
$$

$$
\left\{\begin{array}{l}
c \cdot N_{i} N_{p} \geq c_{0} \cdot\left(n \cdot N_{i}\right)+c_{0} \cdot m \\
c \cdot N_{i} N_{p}<c_{0} \cdot\left(n \cdot N_{i}\right)+c_{0} \cdot m+W_{p} \cdot N_{i} N_{p}
\end{array}\right.
$$

Condition I is not adequate for generating all laser pulses, as pulses appear when $c \geq$ $W_{p}$, rather than the whole scanning period. Pulses that should appear before $W_{p}$ can be determined by the following extra condition.

Condition II: $\mathrm{t}_{\mathrm{n}} \leq c+c_{0}<t_{n}+W_{P}$, which can be expanded as

$$
\left\{\begin{array}{l}
c \cdot N_{i} N_{p}+c_{0} \cdot N_{i} N_{p} \geq c_{0} \cdot\left(n \cdot N_{i}\right)+c_{0} \cdot m \\
c \cdot N_{i} N_{p}+c_{0} \cdot N_{i} N_{p}<c_{0} \cdot\left(n \cdot N_{i}\right)+c_{0} \cdot m+W_{p} \cdot N_{i} N_{p}
\end{array}\right.
$$

Logically, all laser pulses can be generated by

$$
\text { Output }=\text { Condition I } \| \text { Condition II }
$$

where $\|$ denote or operation between condition I and condition II.

\subsubsection{Implementation on a Field Programmable Gate Ar- rays (FPGA)}

The above analysis is very easy to understand, however, it is not the most elegant way to implement it on a FPGA because a lot of calculations are involved. Instead, a counter can be used to control the width of a laser pulse. In this way, only the moment of the rising edge of each laser pulse is required to calculate:

$$
c \cdot N_{i} \cdot N_{p} \geq n \cdot\left(c_{0} \cdot N_{i}\right)+c_{0} m
$$

Namely, the FPGA core only needs to keep checking whether (3.27) is satisfied; when this condition is satisfied, the counter for each laser pulse is triggered, and it is cleared when the specified number of ticks is over.

The term $n \cdot\left(c_{0} \cdot N_{i}\right)$ is calculated in parallel to generate $N_{p}$ laser pulse triggers by

$$
\left\{\begin{array}{c}
\text { Left } \geq 1 \cdot\left(c_{0} \cdot N_{i}\right)+c_{0} m \\
\text { Left } \geq 2 \cdot\left(c_{0} \cdot N_{i}\right)+c_{0} m \\
\text { Left } \geq 3 \cdot\left(c_{0} \cdot N_{i}\right)+c_{0} m \\
\quad \vdots \\
\text { Left } \geq N_{p} \cdot\left(c_{0} \cdot N_{i}\right)+c_{0} m
\end{array}\right.
$$

where Left $=c \cdot N_{i} \cdot N_{p}$. Indeed, the multiplication outside the bracket in the term $n$. $\left(c_{0} \cdot N_{i}\right)$ can be calculated by adding the product of $c_{0} \cdot N_{i}$ by $n$ times, so that $n$ multipliers can be saved. Therefore, only 4 multipliers are needed in total. The transfor- 
mation above simplifies the design of the FPGA core program significantly, since floating point calculations are completely avoided and very less multipliers are needed. Therefore, the resource consumption of the FPGA does not increase dramatically with increasing the number of laser pulses. So far, $1 \sim 16$ pulses are available to be set up by a user.

The 5 laser pulse scheme of the FPGA core realizing the above algorithm is shown in the following scheme

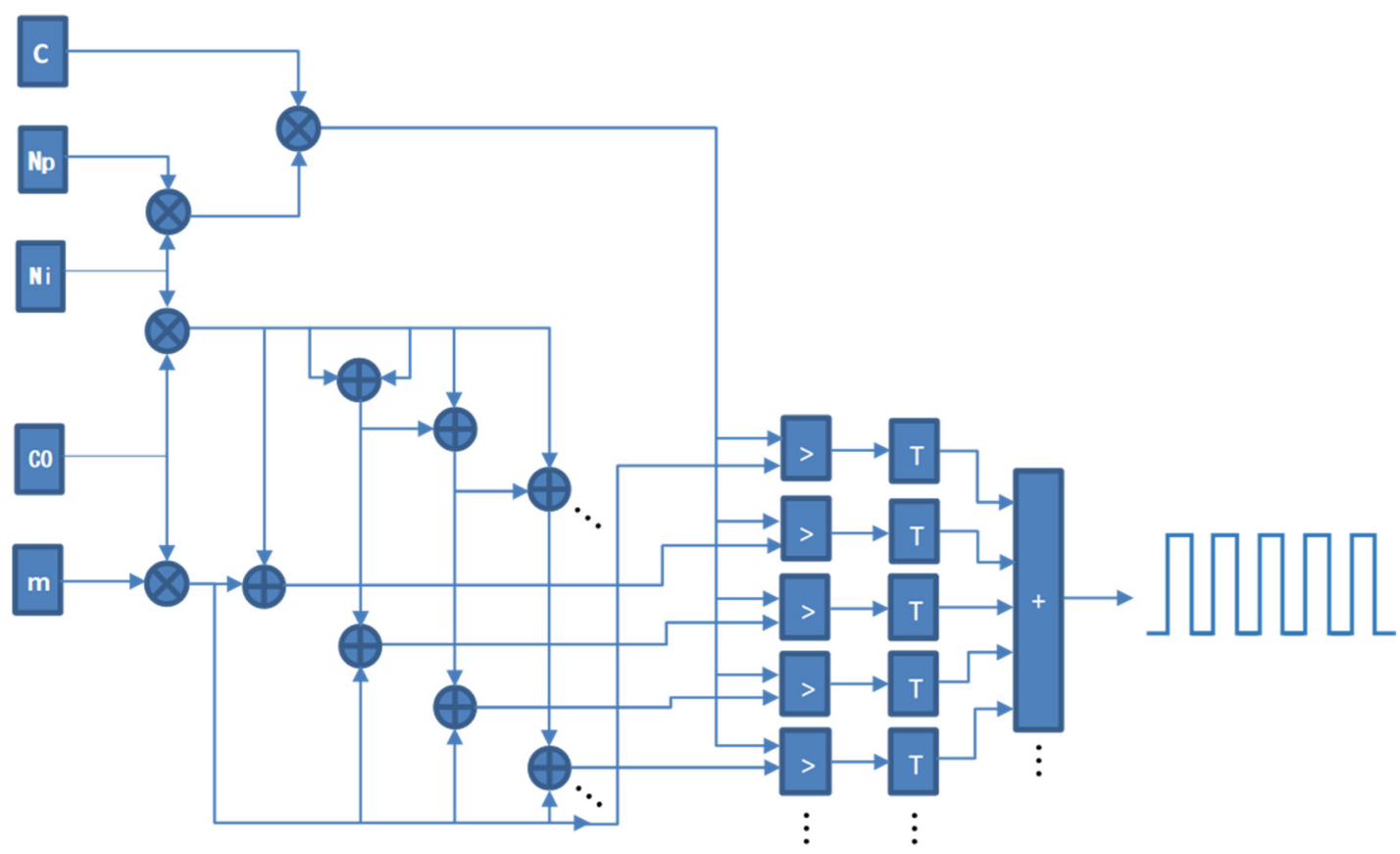

Figure 3.6 Counter $\mathrm{C}$ is used to measure the cycle of spinning-disk-output signal, which is around $3 \mathrm{~ms}, \mathrm{C} 0$ is the register used to store the measured value $\mathrm{C}, \mathrm{m}$ is used to indicate the shifting offset of laser pulses for the $(m+1)^{\text {th }}$ frame.

A global counter of the rising edge of the spinning-disk-output signal is used to control the number cycles of the spinning-disk-output signal for a frame, for the camera trigger pulse generation, and for the increment time of register $m$. The signal synchronization and pulse generation by the counters is very easy to implement on an FPGA, the designed signals can be seen clearly in the waveforms shown in the next section.

\subsubsection{Simulation}

The whole code is written by Verilog HDL language [53], a very strong hardware description language. The code will be published in a special paper dedicated to the whole SCD-ISM hardware and software, an the software package will be released in this connection.

In order to verified my design, the algorithms and its implementations was tested by a Testbench simulation [54]. The core function is written with the Verilog HDL language, 
the input and output interface of the module are defined as shown in Fig. 3.7. The test is implemented with the help of Modelsim SE 10.5 software [55].

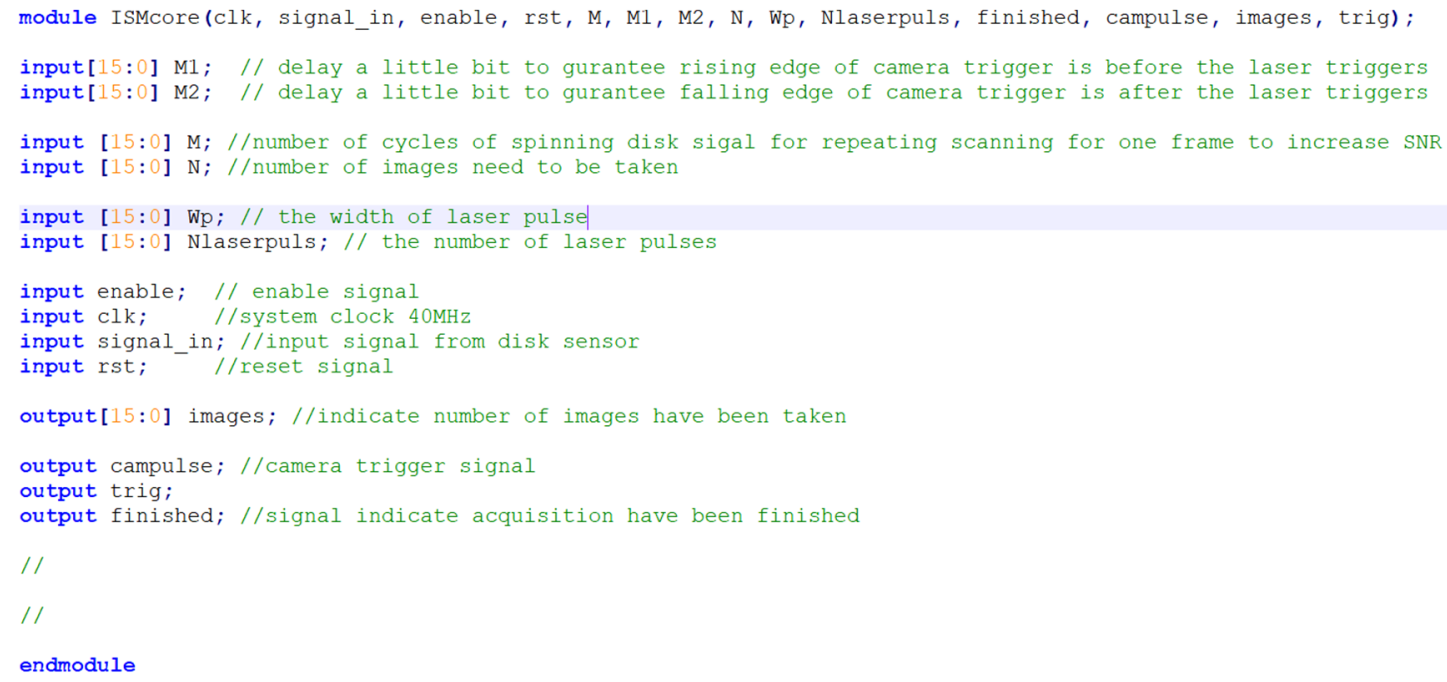

Figure 3.7 FPGA core interface

The spinning disk signal is simulated by generating a rectangle waveform with a frequency of $300 \mathrm{~Hz}$, and a $0.5 \mu$ s error of the cycle time s is introduced to simulate the real experimental conditions. In the following, I present several test cases with different input values.

\section{Case 1:}

Number of images $N=10$, number of scan cycle repeats $M=1$, number of laser pulses Nlaserpuls $=16$, and laser pulse width $6 \mu$ s. All input parameters were set in the above FPGA core interface. The simulation result is shown in Fig. 3.8.
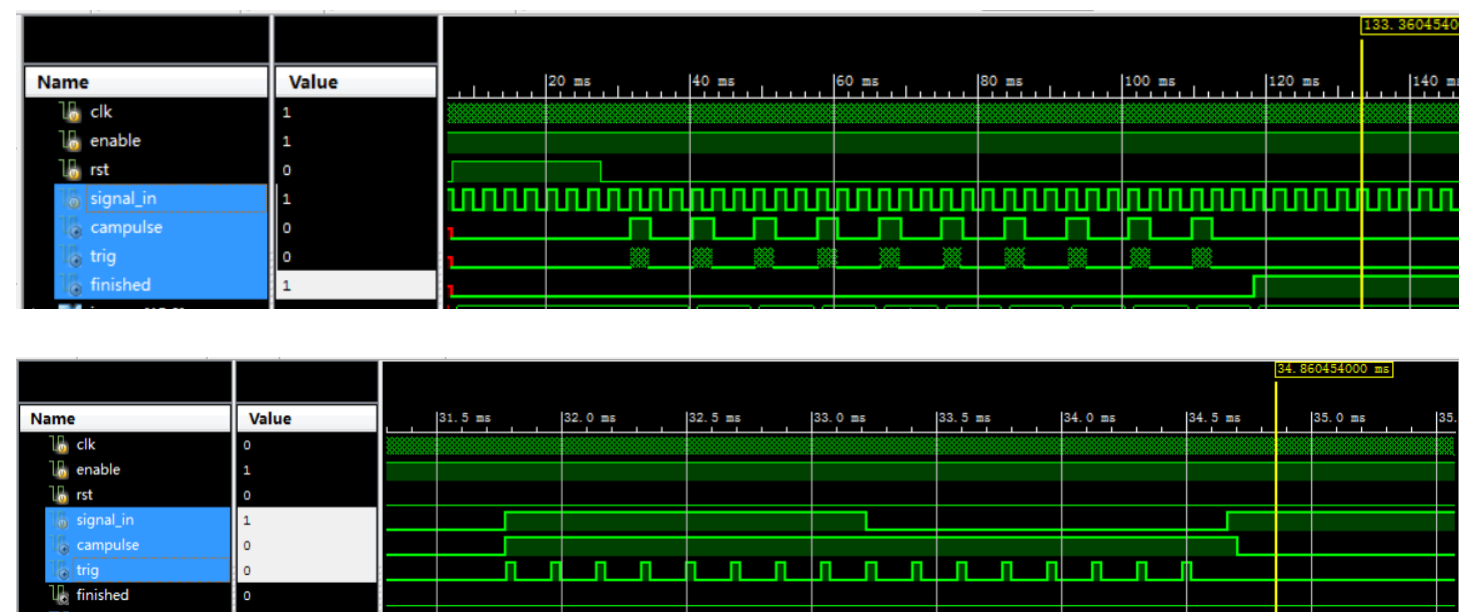

Figure 3.8 Top: the whole period to acquire a set of ISM raw image Zoom-in, down: signal within single scanning period.

\section{Case 2:}

Number of images $\mathrm{N}=9$, scan cycle repeats $\mathrm{M}=2$, number of laser pulses 
Nlaserpuls $=8$. The width of a laser pulse and all other parameters were the same as in case 1 , and will be kept the same also for all following test cases.

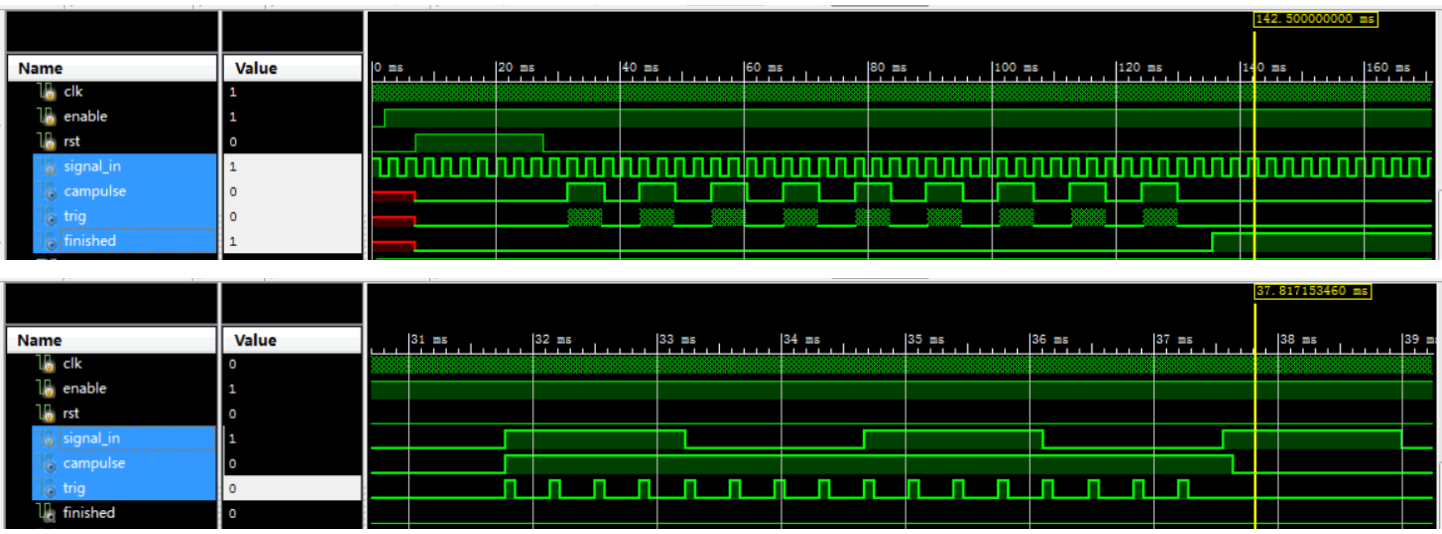

Figure 3.9

\section{Case 3:}

Number of images $\mathrm{N}=12$, scan cycle repeats $\mathrm{M}=2$, number of laser pulses Nlaserpuls $=6$.

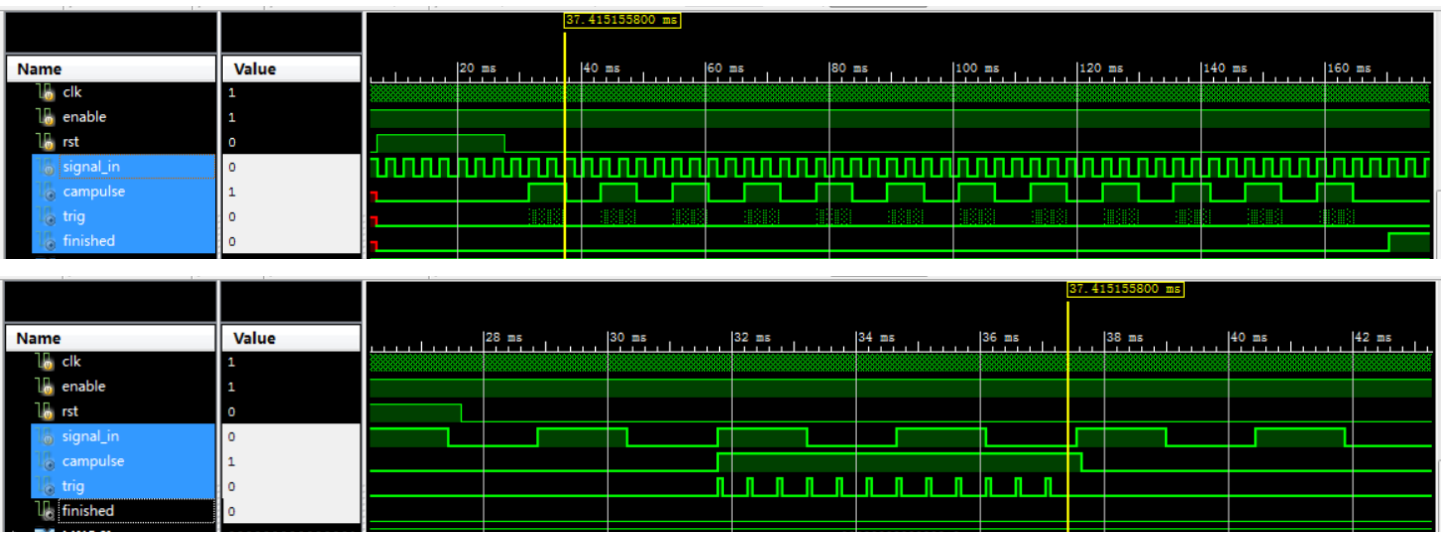

Figure 3.10

\section{Case 4:}

Number of images $\mathrm{N}=8$, scan cycle repeats $\mathrm{M}=3$, number of laser pulses Nlaserpuls $=5$.
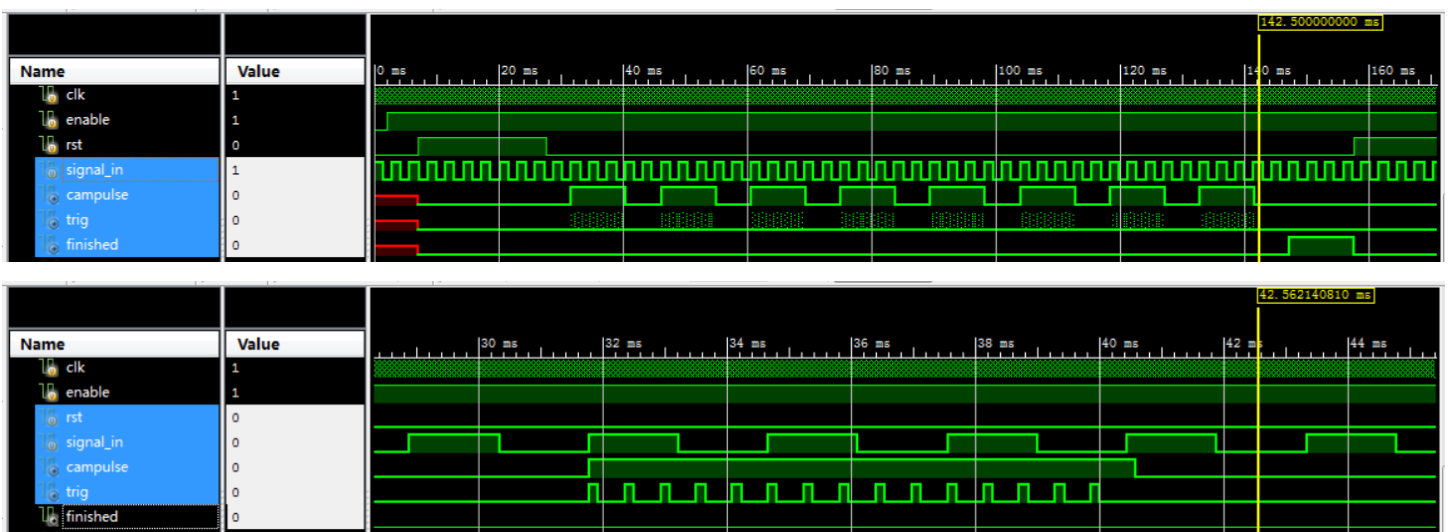

Figure 3.11

\section{Case 5:}

Number of images $\mathrm{N}=7$, scan cycle repeats $\mathrm{M}=5$, number of laser pulses Nlaserpuls $=4$ 

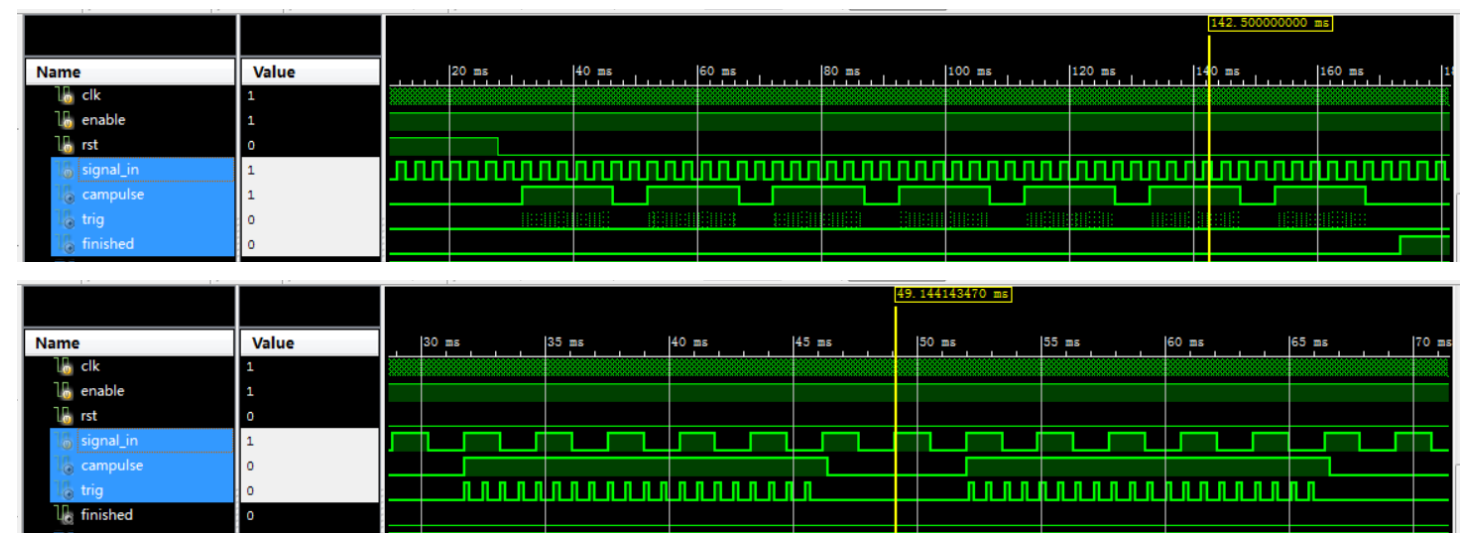

Figure 3.12

\section{Case 6:}

Number of images $\mathrm{N}=8$, scan cycle repeats $\mathrm{M}=5$, number of laser pulses Nlaserpuls $=4$.

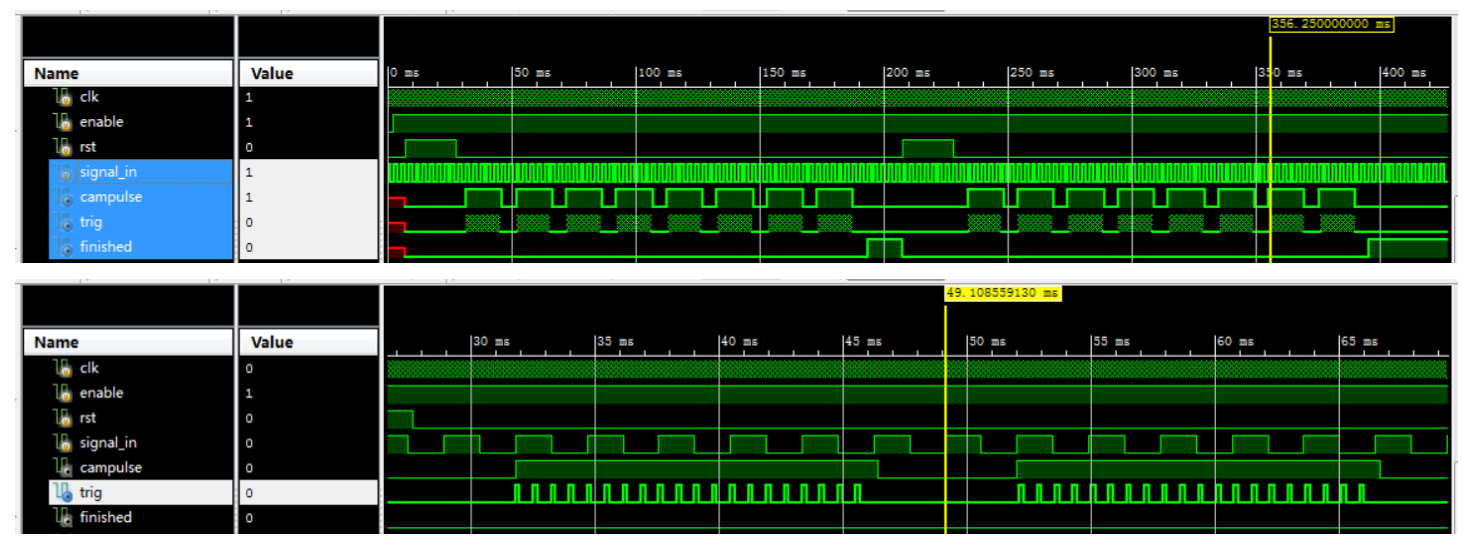

Figure 3.13

The simulation results show that the generated pulse signals are in perfect agreement with original design values.

\subsection{Acquisition GUI based on $\mu$ Manager Plugin}

The design of the data acquisition GUI is based on the very popular open source software $\mu$ Manager that was developed for bio-imaging. With $\mu$ Manager, it is very easy to integrate new device functions and to extend functions of an existing setup.

The main component is a $\mu$ Manager plugin written in Java and $\mathrm{C}++$ adapted to the application interface (API) guidelines for $\mu$ Manager plugin development. The FPGA controller can be controlled via the user interface of the $\mu$ Manager plugin. One major benefit of the $\mu \mathrm{M}$ software is its flexibility and support for various cameras and microscope equipment for which $\mu$ Manager device adapters exist. In addition to the controller, also a plugin for image reconstruction is included in the software. After setting a few 
necessary input parameters, image acquisition requires only one click. The graphical user interface (GUI) of the plugin for image acquisition is shown in Figure 3.14.

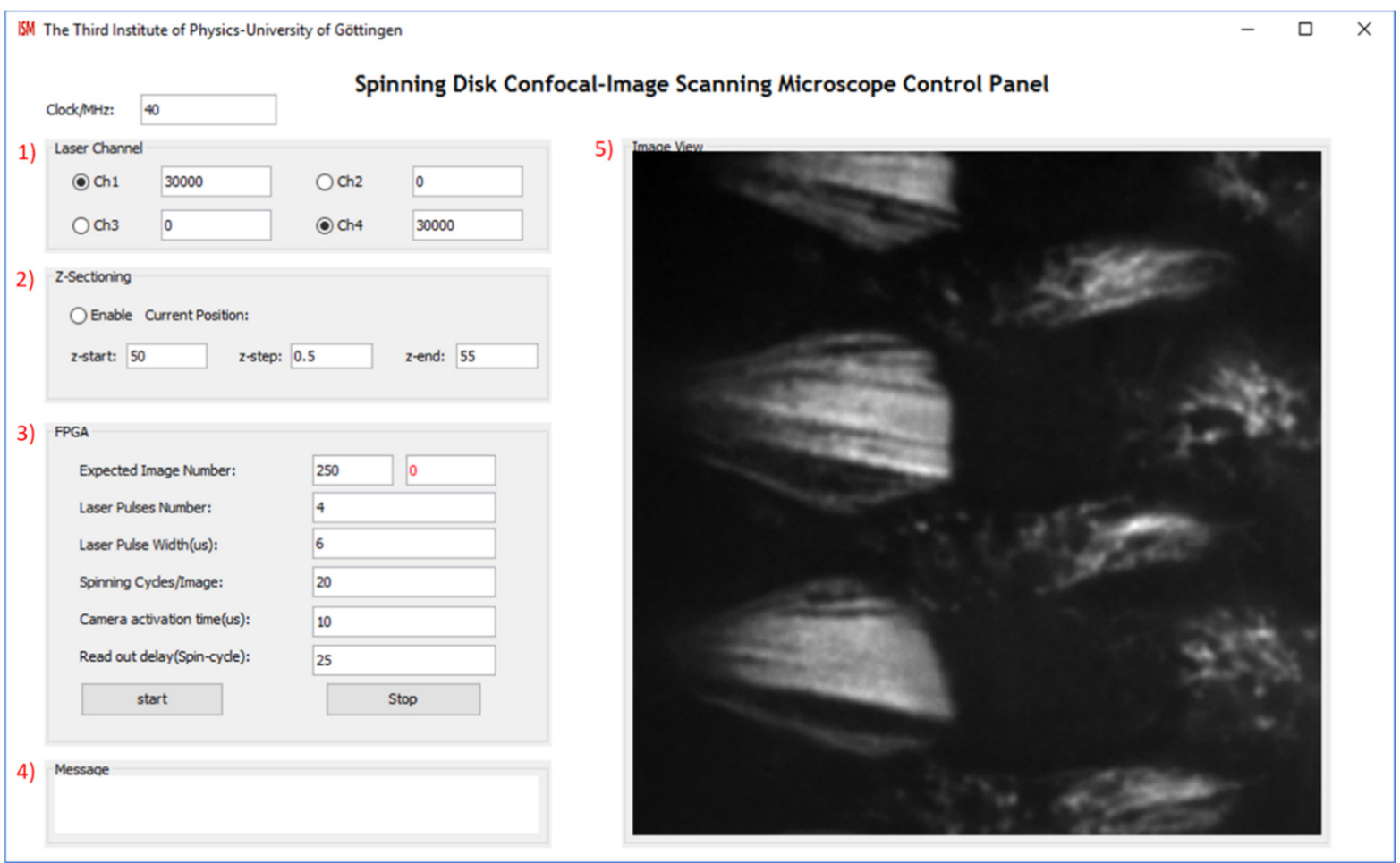

Figure 3.14 Acquisition GUI for SCD-ISM system.

In the ISM control panel, there are five main panels: 1) laser color control panel, 2) zsectioning control panel, 3) FPGA control panel, 4) message viewer, and 5) image viewer.

In the laser color control panel (1), the intensity of the four AOTF color channels can be controlled between 0 and $100 \%$. Select any channel by clicking the radio button.

The z-sectioning panel (2) supports z-sectioning acquisition if the toggle box is checked. Values for the start position, step size, and the end position (in $\mu \mathrm{m}$ ) can be set by this panel. The current focus position will be shown when the mouse pointer hovers over the panel, which helps to define these parameters.

The FPGA control panel (3) contains all settings for the FPGA control core. The number of images $N_{\text {ISM }}$ (scan positions), the number of laser pulses $N_{\mathrm{P}}$ per cycle, the duration of laser pulses (in $\mu \mathrm{s}$ ), the waiting time (in $\mu \mathrm{s}$ ) for camera activation (from the rising edge of the camera trigger to the rising edge of laser pulse), the readout delay of camera (from the end of last laser trigger to the end of camera trigger, in multiples of spinning-disk scan cycles).

In the message box (4), acquisition information and errors will be shown. This gives the user good control over the whole process of acquisition. 
When the acquisition is finished, the sum of all frames will be shown in the image viewer (5).

\subsubsection{Image Acquisition}

In case all the devices are ready, image acquisition can be carried out by the following steps:

1) Start and check all devices and make sure all devices are ready.

2) Start the $\mu$ Manager software and place your sample on the microscope. Focus on the sample, for example by using the "live" imaging mode of $\mu$ Manager to use the SCD without ISM. Make sure that the triggering mode of the camera and of the AOTF are set to "Internal", "Software" or equivalent. Adjust the laser power.

3) Start the SCD-ISM plugin from the "Plugins" menu in $\mu$ Manager. NOTE: If you use a different camera than an Andor or Hamamatsu camera, you need to set the external triggering mode of your camera appropriately in the $\mu$ Manager's "Device Property Browser".

4) Set the AOTF mode as "external" in the app from the AOTF's manufacturer. Select the laser channel and set the intensity level.

5) In the z-sectioning panel, un-check the toggle box to do single slice acquisition or check it to do z-sectioning acquisition. For z-sectioning acquisition, the values for the start of z-position, step size, and the end of z-position (absolute coordinates in $\mu \mathrm{m})$ should be given.

6) Adjust the parameters in the FPGA control panel to your needs or to the settings you used for the calibration image. Some typical parameters are set to default values. To adjust these parameters, the following should be taken into consideration: 1) there is always a tradeoff between the number of images per slice and the number of laser triggers used. Generally, the more laser triggers are used, the less number of images per slice is required. Even though using a larger number of laser triggers can accelerate acquisition, as more scan points become available in a single image scan period and therefore less frames are required, it can cause overlapping between measured light spots, which impairs the resolution of an ISM image. Our experiments show that 4, 6 and 8 laser pulses and 200 250 frames are optimal. 2) The value of the laser pulse width depends on the speed of the spinning disk-typically, it can be set to $5 \sim 7 \mu \mathrm{s}$. 3) For higher resolution and contrast, a larger number of cycles per image should be used. 4) A minimum value of readout delay should be used to speed up the acquisition. NOTE that the allowed range of laser pulses is 1 to 16 , and the maximum waiting time for camera activation is $20 \mu \mathrm{s}$.

7) Launch the acquisition by simply clicking the start button. If all devices work well, a window displaying the acquired images will be shown. Wait until the acquisition process is finished. In case that acquisition should be aborted, click the stop button to stop the ongoing acquisition. Otherwise, the acquisition session will stop automatically when the expected number of images are taken.

8) Now you can save the image stack by clicking the save button in the bottom of the acquisition window. Alternatively, the "Save images" functionality in the Multi- 
Dimensional Acquisition window can be used to directly save the data to a local drive.

9) A fast way to see if the acquisition was successful is to compute the average intensity image (in ImageJ, go to Image $\rightarrow$ Stacks $\rightarrow$ Z-Project... and choose "Average Intensity" as projection type), which should look like a normal SCD image.

\subsubsection{Image Reconstruction Software}

Image reconstruction is implemented by software developed in our group. In our software, an easy-to-use GUI is built for ISM image processing. The user just needs to give a few parameters and the directory of the ISM raw image stack to calculate an ISM image. The main function contains: 1) ISM image calculation. 2) Average image calculation. 3) Save image in any other format. 4) BAT processing of image stacks. 5) Localizing all light spots in a measured reference raw image.

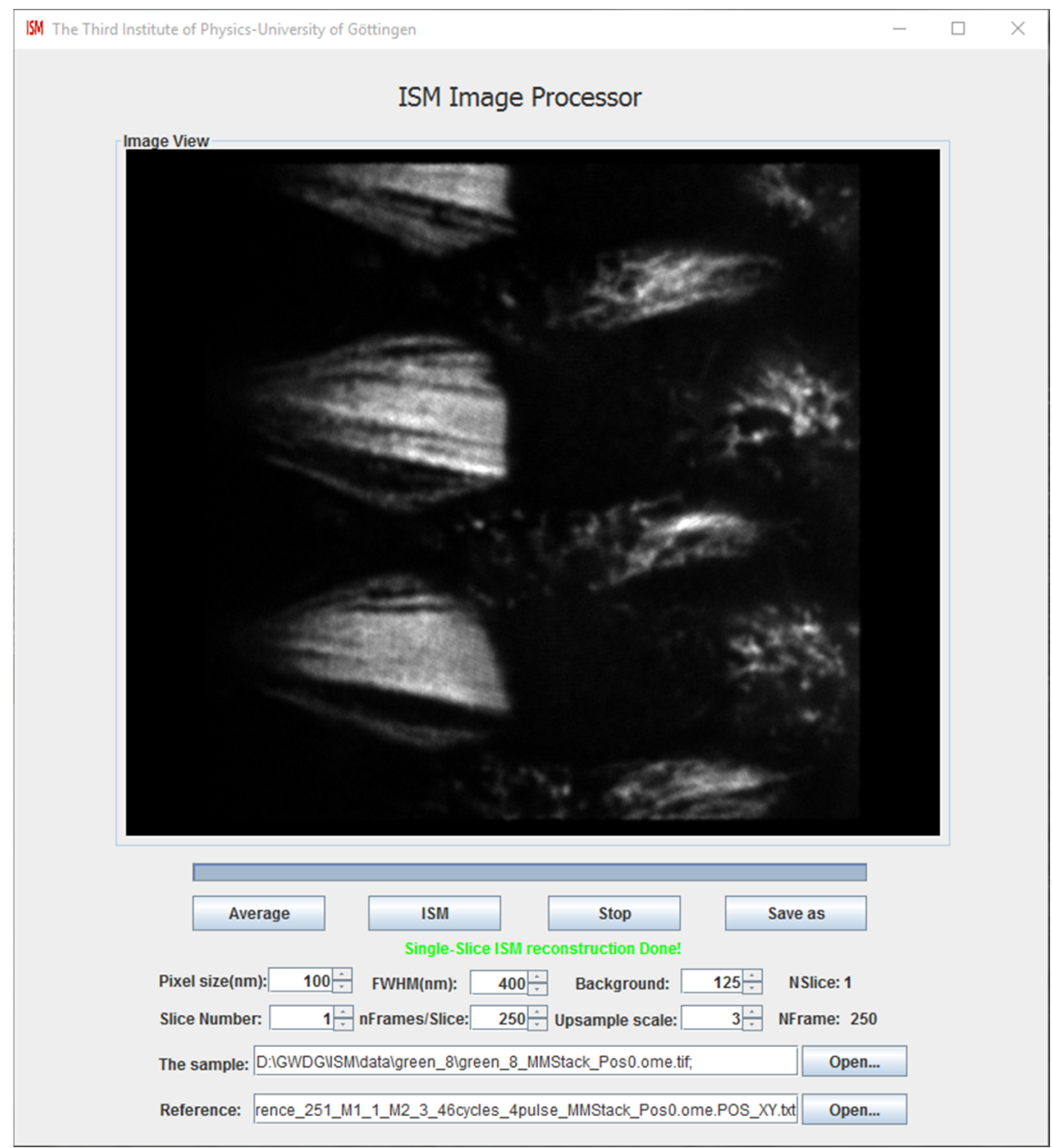

Figure 3.15 Reconstruction GUI

\section{A. Launch the GUI}

Double-click the software file "ISMPRO.exe" or "ISMPRO.jar" to launch the GUI for ISM reconstruction. I made this app also as a Fiji plugin, so if the user would like to use Fiji, he/she just needs to copy the SCD_ISMpro-0.1.0.jar file to the "/plugins" 
folder under the directory of Fiji and launch this GUI from the plugin menu of Fiji. NOTE: running ISMPRO.exe and ISMPRO.jar requires version of JER 1.8.0_161 or higher. Fiji plugin requires Java 8 version of Fiji.

\section{B. Localization}

Basically, localization can be done with two existing popular software packages: RapidSTORM and ThunderSTORM. Our software is able to read the position data of each confocal light spot from the export file by the above-mentioned software. It is also possible to do efficient localization by the embedded localization engine in our software when the signal-to-noise ratio is high (the peak value of measured light spot $>1000$ ). Note: among the exported file formats of ThunderSTORM, only csv files are supported by our software.

\section{ISM image calculation}

1) Input a proper value of pixel size (in nm), FWHM of PSF (in nm), background, the number of slices and the number of frames in each slice and the image up-sampled factor. Depending on the FWHM of a confocal light spot, we recommend that the upscaling factor should be between 4 and 8 to avoid artifacts in the ISM image. NOTE: if the input numbers of slices and frames are not correct, the computation of the ISM reconstruction will not be able to launch. Note: the pixel size input in our software has to be identical to one input in RapidSTORM or ThunderSTORM.

2) Select a raw image stack file of sample by clicking the open button on the right side. In case of 3D ISM calculation, if the stack image is so volume that it is divided into several parts (4GB limited for each part) by $\mu$ Manager, one just needs to select the first stack file. Select the reference file that matches the raw image of a sample. The reference file for ISM calculations supports txt, csv and tif file format. If a txt or csv file is selected, the software will directly read the position data of all light spots from the text file and will calculate the ISM image. If a tif file is selected, the software will first do the localization by the embedded localization engine, and then use the generated text file to calculate an ISM image.

3) Click ISM button to start ISM image calculation. There may be some delay before starting the calculation due to loading the volume raw image data. The reconstruction algorithm can be very heavy for large size of image processing; therefore, it may take from minutes to hours, which depends on the size of image and the upsampling scaling factor used. The progress of processing is visualized by displaying a progress bar and showing in the panel the number of slices and frames that have been processed. When the calculation is finished, the result will be saved as a tif file in the same directory automatically.

\section{Average image calculation}

The steps of calculating Average is very similar to many steps of calculating ISM. Basically, the user needs to input the exact number of slices for z-sectioning and the number of frames for each slice, select the raw image file of a sample, and click Average button. When the calculation is finished, the result will be saved as a tif file in the same directory automatically. 


\section{E. Save image as any other format}

The ISM image reconstruction software supports saving an image in many other popular image formats, such as tif, png, jpg etc. The user just needs to click the Save As button after the average image or ISM image calculation is done, give a full file name in the pop-up dialogue window, and then the image will be saved to the specified folder in the indicated image format.

\section{F. Batch file processing}

Select multi tif file of sample in the same folder from the dialogue window or just copy the absolute path of multi tif file of sample to the text field. The other steps to calculate an average image and an ISM image are the same as mentioned above.

\subsection{Confocal Light Spots Detection and Localization}

The confocal light spots have to be localized before ISM reconstruction. The localization and realignment of the confocal light spots have a great impact to the resolution of a finally reconstructed super-resolved ISM image. The first step is confocal light spots detection. In practice, the signal-to-noise ratio of the confocal light spots of raw images of a real sample are usually dynamic, which is really a disadvantage for accurate detection. Even very low signal-to-noise-ratio light spots can contribute photons to a final ISM image. Therefore, poor detection affects the final image quality. In order to avoid negative detection, a reference image can be measured with a relatively homogenously emitting sample, which can be made from a buffer solution with high label concentration, for confocal light spot detection and localization. The localization information can then be used for ISM image reconstruction. Given a reference image, the confocal light spots can be detected through a correlation between the reference images with an estimated Gaussian function. The correlation function between two vectors is defined by

$$
R(X, Y)=\frac{\sum_{k=1} X_{k} \cdot Y_{k}}{\sqrt{\sum_{k} X_{k}^{2}} \cdot \sqrt{\sum_{k} Y_{k}^{2}}}
$$

For image correlation, the above function is called to calculate the correlation between the template $U$ and the sub-image of an object. The drawback of (3.29) is that it has to go through all pixels to calculate the complete correlation function. In fact, the correlation function between a template and an image can be written by

$$
R(U, I)=\frac{U \circledast I}{c \sqrt{W \circledast I^{2}}}
$$

where $\circledast$ denotes correlation between two images, $\mathrm{c}=\sqrt{\sum U_{k}^{2}}$, which just need to be calculated once, $\mathrm{W}$ denotes the mask function that indicates $U>0$. This is indeed a fast algorithm to calculate image correlation as the image correlation $\circledast$ can be calculated by FFT. The detection then can be realized by finding the local maximum pixels, 
which indicate the rough position of the confocal light spots. Finally, the exact position can be determined by an accurate Gaussian fitting algorithm, which will be studied in the next chapter.

\subsubsection{Simulation}

For simulation, Gaussian images are generated with $\sigma=3$, random peak intensity in the range $[30,100]$, and a background of 100 . The ground-truth image and the noisy image with Poisson noise are shown in Fig. 3.16.
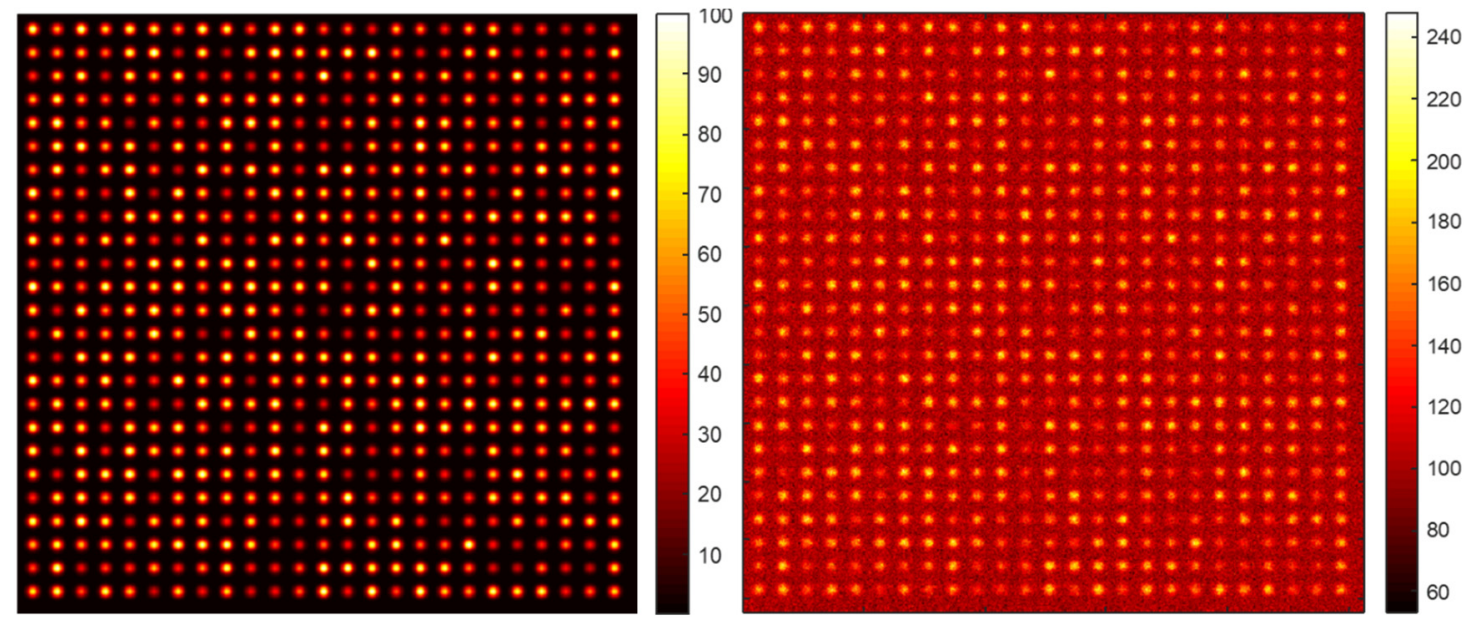

Figure 3.16 Left: ground-truth. Right: noisy image.

In detection algorithm, a Gaussian function is used as the template for correlation calculation. An extra step is introduced to estimate the sigma value of the template Gaussian function. Based on a proper guessed sigma, all light spots are firstly detected with a relatively higher threshold value (e.g. 0.7 ), then all the detected light spots are fitted with Gausian function model to obtain their sigma values. Finally the sigma value of the template Gaussian function is determined by taking the average of the resulted sigma values by fitting. The correlation function between the above noisy image with a well-estimated normalized Gaussian function and the detection result are shown in Fig. 3.17.
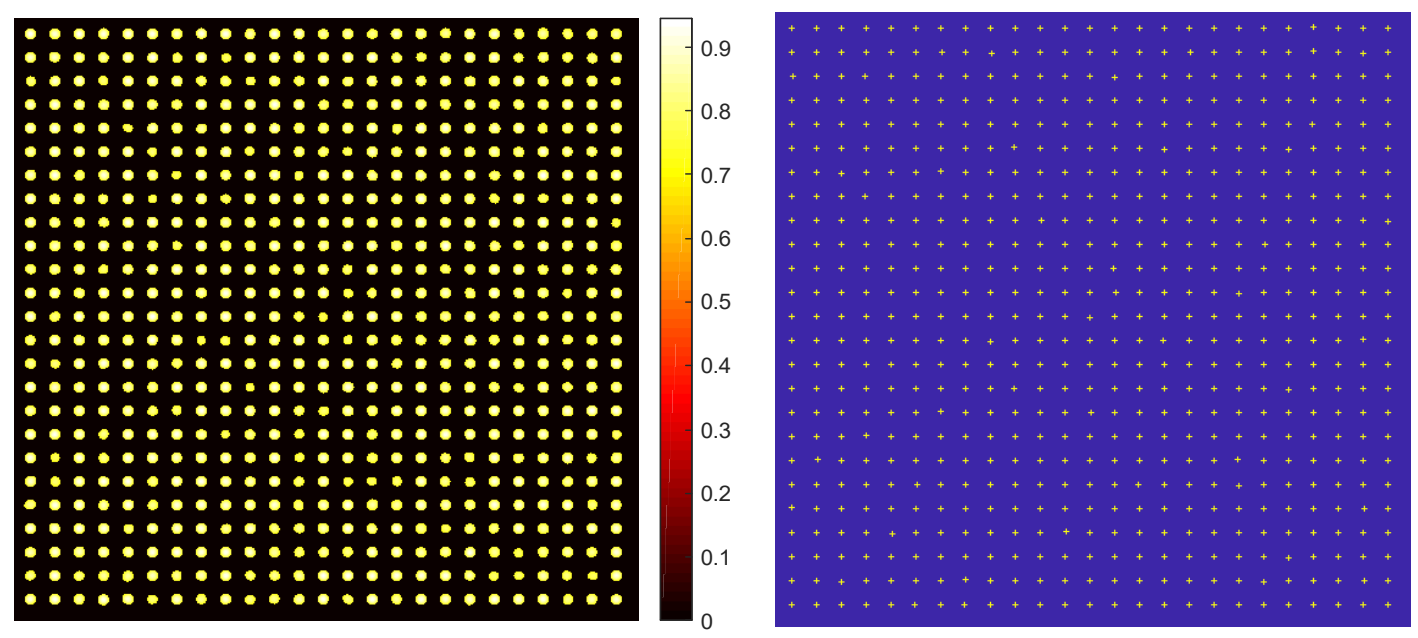

Figure 3.17 Left: correlation $($ Corr $>0.7)$. Right: position markers 
The simulation shows that the observed image used for fitting is very noisy, and some candidates are so dim that they challenge existing localizers, even the ThunderSTORM software fails to detect all of them. Fig 3.18 shows the result of ISM image reconstruction with the detection by proposed algorithm and ThunderSTORM software.

(a)

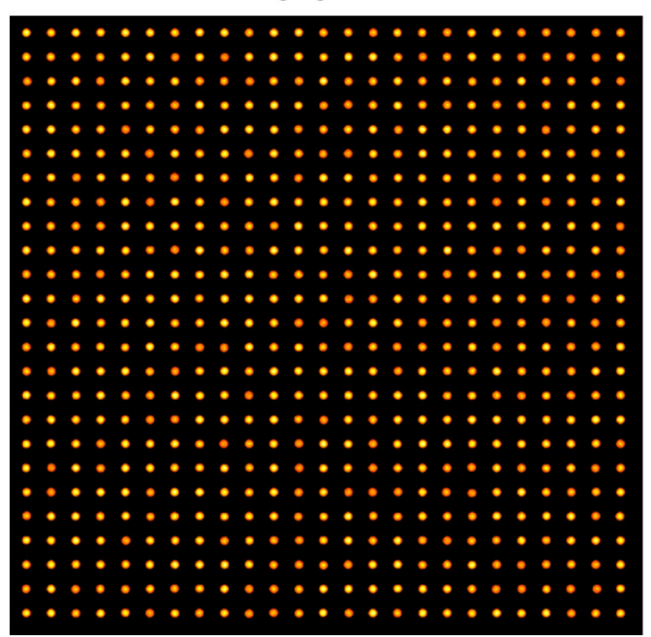

(b)

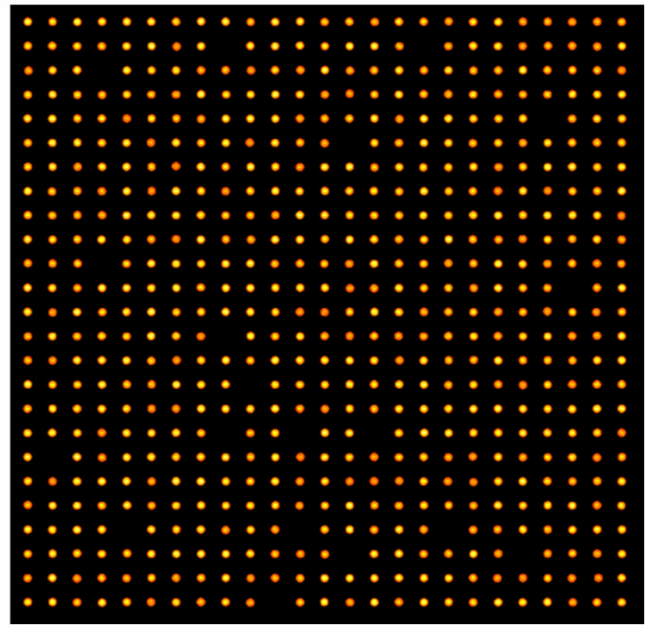

Figure 3.18 (a) ISM with the proposed detection algorithm (b) ISM image with localization data generated by ThunderSTORM software

By the proposed correlation calculation, the resulting correlation functions are quite homogenous, which is very beneficial for detecting the measured confocal light-spots. A large number of simulations show that a $100 \%$ correct detection can be achieved for peak intensity values of $I_{\max }>30$.

\subsection{Discussion}

In this chapter, I illustrated the principle of ISM and showed how ISM generates superresolution. I introduced the SDC-ISM system and described the image reconstruction of ISM. I analyzed the efficient calculation formula executed on FPGA for SDC-ISM pulsing and designed a FPGA core for generating pulses for synchronizing the spinning disk, the camera, and the laser, which is crucial for SDC-ISM. I verified the FPGA design with simulations, which guarantees that the functional design is correct. Based on the $\mu$ Manager software, I developed an easy-to-use software for image acquisition and image reconstruction. Finally, I studied confocal light detection methods for accurate localization. 


\section{Chapter 4}

\section{Accurate Gaussian}

\section{Fitting}

\subsection{Introduction}

Gaussian fitting is very commonly used in many applications, such as single-molecule localization (PhotoActivated Localization Microscopy (PALM) [12], Stochastic Optical Reconstruction Microscopy (STORM) [56]), or Image Scanning Microscopy (ISM) $[18,33]$, or Gaussian parameter estimation [57] and analysis of atomic resolution images [58], etc. So far, there have been several methods proposed for 2D Gaussian fitting. Basically, all existing methods can be classified into two types: polynomial fitting, and accurate fitting.

Polynomial fitting is based on the fact that a Gaussian function is the exponential of a quadratic function. Therefore, it can be converted into a polynomial function by applying the logarithm, and then each parameter of the Gaussian function can be found from the coefficients of a polynomial fit. The basic Gaussian fitting technique of this type is Caruanas' algorithm [59], which is computationally very efficient. However, its accuracy can be seriously affected by noise. A modified version of Caruanas' algorithm has been proposed in Ref. [60], which improves the noise robustness of the fitting algorithm and results in a better fitting accuracy. However, any constant offset is ignored by this algorithm. Therefore, a baseline correction is always required to improve the fitting precision [57], otherwise, its accuracy will be affected by noise and the background of the measured data. In an experiment, however, background does always exist and is very difficult to precisely estimate. For this reason, these types of methods is not suitable for accurate fitting, such as accurate Gaussian parameter estimation and localization.

Accurate fitting methods are mainly based on an accurate fit model and an effective iterative algorithm, such as a Least-Square (LSQ) optimization method or a Maximum Likelihood (ML) estimator. In fact, algorithms using these techniques have been implemented in RapidSTORM [61], which is a well-known open source software for singlemolecule localization fluorescence microscopy. This technique is undoubtedly very powerful. However, the algorithm involves calculating Hessian matrices, therefore implementation of this algorithm is not easy and the computational load is very heavy.

Even though there are many existing nonlinear optimization solvers available that could be used for 2D Gaussian fitting, for example the Matlab optimization toolbox, most of existing solvers are platform-dependent. In addition, transferring a potential solver to a customized platform is not convenient, because the existing solvers usually depend on a lot of other libraries, platforms, and even operation systems.

In this chapter, I study two efficient Gaussian fitting algorithms based on LSQ and ML 
for accurate fitting of the focus positions in ISM. In part 2, I describe a model of Gaussian fitting based on LSQ and derive the gradient of the objective function. In part 3, I analyze the Gaussian fitting based on ML. In part 4, I verify the fit algorithms and study some factors that affect their performance. In part 5, I summarize my work.

\subsection{Least-Square Based Fitting Algorithm}

Least Square Fitting searches for a solution that minimizes the sum of the squares of the error between a model function and measured data [62]. For Gaussian fitting, the model function is a 2D Gaussian function that can be written as

$$
f(\theta)=A e^{-\frac{(x-a)^{2}+(y-b)^{2}}{2 \sigma^{2}}}+C
$$

where $A$ is the amplitude, $(a, b)$ are the coordinates to be estimated for localization, $\sigma$ is a width parameter, and $C$ denotes the background.

For Least Squares Minimization, the error function is defined as

$$
E(\theta)=\sum_{i}\left[f(\theta)_{i}-z_{i}\right]^{2}
$$

where $z$ is the measured data. Minimizing this goal function results in the solution for the fit parameters

$$
\hat{\theta}=\underset{\theta}{\operatorname{argmin}} E(\theta)
$$

Computationally, an analysis of the gradient is very important for efficiently solving (4.3). The derivatives of the goal function after each parameter can be written as

$$
\left\{\begin{array}{l}
\frac{\partial E}{\partial A}=2[f(\theta)-z] \cdot e^{-\frac{(x-a)^{2}+(y-b)^{2}}{2 \sigma^{2}}} \\
\frac{\partial E}{\partial a}=2[f(\theta)-z] \cdot A e^{-\frac{(x-a)^{2}+(y-b)^{2}}{2 \sigma^{2}}} \cdot \frac{x-a}{\sigma^{2}} \\
\frac{\partial E}{\partial b}=2[f(\theta)-z] \cdot A e^{-\frac{(x-a)^{2}+(y-b)^{2}}{2 \sigma^{2}}} \cdot \frac{y-b}{\sigma^{2}} \\
\frac{\partial E}{\partial \sigma}=2[f(\theta)-z] \cdot A e^{-\frac{(x-a)^{2}+(y-b)^{2}}{2 \sigma^{2}}} \cdot \frac{(x-a)^{2}+(y-b)^{2}}{\sigma^{3}} \\
\frac{\partial E}{\partial C}=2[f(\theta)-z]
\end{array}\right.
$$

During computation, the values of the common terms $(x-a)^{2}+(y-b)^{2}$ and $e^{-\frac{(x-a)^{2}+(y-b)^{2}}{2 \sigma^{2}}}$ can be stored in memory to improve efficiency.

When recognizing that $\sigma^{2}$ can be regarded as a single variable, the model above can be simplified. The model function can be rewritten as

$$
f(\theta)=A e^{-\frac{(x-a)^{2}+(y-b)^{2}}{2 \sigma}}+C
$$


and the gradient of the objective function can be rewritten as

$$
\left\{\begin{array}{l}
\frac{\partial E}{\partial A}=2[f(\theta)-z] \cdot e^{-\frac{(x-a)^{2}+(y-b)^{2}}{2 \sigma}} \\
\frac{\partial E}{\partial a}=2[f(\theta)-z] \cdot A e^{-\frac{(x-a)^{2}+(y-b)^{2}}{2 \sigma}} \cdot \frac{x-a}{\sigma} \\
\frac{\partial E}{\partial b}=2[f(\theta)-z] \cdot A e^{-\frac{(x-a)^{2}+(y-b)^{2}}{2 \sigma}} \cdot \frac{y-b}{\sigma} \\
\frac{\partial E}{\partial \sigma}=[f(\theta)-z] \cdot A e^{-\frac{(x-a)^{2}+(y-b)^{2}}{2 \sigma}} \cdot \frac{(x-a)^{2}+(y-b)^{2}}{\sigma^{2}} \\
\frac{\partial E}{\partial C}=2[f(\theta)-z]
\end{array}\right.
$$

This transformation simplifies the calculation and optimization algorithm since it reduces the non-linearity of the above model.

After building the fitting model, the next problem is to minimize the goal function. The basic non-linear optimization method is Newton's method. The drawback of Newton's method is that it requires the calculation of the $2^{\text {nd }}$ order derivative of the objective function, which is very complicated and computationally costly. The simplest alternative to Newton's method is the Gauss-Newton method [63], which abandons the second order derivative in the Hessian matrix and uses only the first order derivative to approximate Newton's method. Therefore, it simplifies the iteration formula and significantly reduces the computational complexity, when compared to Newton's method. Another popular technique, the Levenberg-Marquardt method [64, 65], which is actually a hybrid of the Steepest Descend method [66] and the Gauss-Newton method, is also a good candidate for non-linear optimization, but it is still not as efficient as the Gauss-Newton method. Due to its simplicity and efficiency, I choose here the Gauss-Newton method to implement the following optimization algorithm.

The iterative formula of the Gauss-Newton method can be written as

$$
p_{k+1}=p_{k}-\left(J^{T} \cdot J\right)^{-1} \cdot J^{T} \cdot r
$$

where $p_{k}$ denotes the estimation of the $k^{\text {th }}$ iteration, $T$ denotes matrix transposition, and $J$ denotes the Jacobi matrix which is defined as

$$
J(p)=\left[\begin{array}{ccc}
\frac{\partial r_{1}}{\partial p_{1}} & \cdots & \frac{\partial r_{1}}{\partial p_{M}} \\
\vdots & \ddots & \vdots \\
\frac{\partial r_{N}}{\partial p_{1}} & \cdots & \frac{\partial r_{N}}{\partial p_{M}}
\end{array}\right]
$$

while $r$ denotes the residual between the observed and estimated values. When inspecting the iteration formula above, it is found that the main problem of implementing the Gauss-Newton method is the calculation of the Jacobi matrix. However, calculating the Jacobi matrix is quite simple, since only first-order derivatives are involved. 
Based on the fit model, we have $p=[A, a, b, \sigma, C]$ and $r=f(\theta)-z$. Using the gradient of the goal function given above, the derivatives of the residuals after each parameter in the Jacobi matrix are calculated by

$$
\left\{\begin{array}{l}
\frac{\partial r}{\partial A}=e^{-\frac{(x-a)^{2}+(y-b)^{2}}{2 \sigma}} \\
\frac{\partial r}{\partial a}=A e^{-\frac{(x-a)^{2}+(y-b)^{2}}{2 \sigma}} \cdot \frac{x-a}{\sigma} \\
\frac{\partial r}{\partial b}=A e^{-\frac{(x-a)^{2}+(y-b)^{2}}{2 \sigma}} \cdot \frac{y-b}{\sigma} \\
\frac{\partial r}{\partial \sigma}=A e^{-\frac{(x-a)^{2}+(y-b)^{2}}{2 \sigma}} \cdot \frac{(x-a)^{2}+(y-b)^{2}}{\sigma^{2}} \\
\frac{\partial r}{\partial C}=1
\end{array}\right.
$$

It is straightforward to implement the Gauss-Newton method if the Jacobi matrix is available.

\section{Algorithm 4.1}

Initialize $p=\left[\begin{array}{lllll}A_{0} & a_{0} & b_{0} & \sigma_{0} & C_{0}\end{array}\right]$, and tolerance $\epsilon$;

while: $\left\|\Delta_{k}\right\| \geq \epsilon$

$$
\begin{aligned}
& r=z\left(p_{k}\right)-z ; \\
& J=\left[\frac{\partial r}{\partial A} \frac{\partial r}{\partial a} \frac{\partial r}{\partial b} \frac{\partial r}{\partial \sigma} \frac{\partial r}{\partial C}\right] ; \\
& \Delta_{k}=-\left(J^{T} \cdot J\right)^{-1} \cdot J^{T} \cdot r ; \\
& p_{k+1}=p_{k}+\Delta_{k} ; \\
& \text { If }\left\|p_{k+1}-p_{k}\right\|<\epsilon
\end{aligned}
$$

Break;

\section{end}

\section{end}

In theory, the Gauss-Newton method is an approximation to the Newton method for least-square problem, and its convergence is always in the fastest direction. Numerous simulations show that it is a very robust and efficient algorithm for solving the Gaussian-fitting problem. It takes only several iterations to converge with a proper estimated initial value of each parameter. The final problem is then the estimation of the initial value of each parameter.

In practice, estimating each parameter of a Gaussian function from the measured data is very easy. For example, the maximum value of the observed data can be used as the initial value of the amplitude $A$; the coordinate of the maximum can be used as the initial value of the position parameters $a, b$, respectively, 


$$
\left\{\begin{array}{l}
A_{0}=\max (z) \\
{\left[a_{0}, b_{0}\right]=\text { indexof }(A)}
\end{array}\right.
$$

where indexof denotes taking the coordinate of the maximum value of the measured data. The initial sigma value can be estimated by using the relationship between the Full-Width at Half-Maximum (FWHM) [67]

$$
\sigma_{0}=\frac{\mathrm{FWHM}}{2.35482}
$$

while the FWHM can be estimated by counting the number of pixels within $z n \geq \max \frac{(z)}{2}$. Since $A_{0}$ has been estimated as just described, it is easier to calculate the FWHM through the data in the column and the row that cross $A_{0}$ along $z$. The initial parameter $C_{0}$ is not as important as the other parameters. It can be either set to $\min (z)$ directly, or estimated by measuring the background value of the detector a priori, if possible.

\subsection{Maximum Likelihood Estimation Based Gauss- ian Fitting Algorithm}

The average value of the detected signal, e.g. the 2D Gaussian function, can be written as

$$
\lambda(\theta)=A e^{-\frac{(x-a)^{2}+(y-b)^{2}}{2 \sigma^{2}}}+C
$$

where $\theta=(A, a, b, \sigma, C)$ is the parameter vector of the Gaussian function. Assuming a Poissonian statistics of signal detection, the chance to record a signal $z=\left\{z_{i}\right\}$ given an average $\lambda$ is given by

$$
P(z \mid \lambda)=\frac{\lambda_{i}^{z_{i}}}{z_{i}} e^{-\lambda_{i}}, i=1,2, \ldots, n
$$

where $z$ is the measured data. The Maximum Likelihood Estimate function can be written as

$$
\begin{aligned}
& L\left(\lambda \mid z_{1}, z_{2}, \ldots, z_{n}\right)=\ln \prod_{i} \frac{\lambda_{i}^{z_{i}}}{z_{i} !} e^{-\lambda_{i}} \\
& =\sum_{i}(z \ln \lambda-\lambda)-\sum_{i} \ln \left(z_{i} !\right)
\end{aligned}
$$

Ignoring the constant term, the objective function maximizing the above likelihood function can be written as

$$
E(\theta)=-L[\lambda(\theta)]=\sum_{i}\left(\lambda_{i}-z_{i} \ln \lambda_{i}\right)
$$

The derivative of this objective function can be written as

$$
E^{\prime}(\theta)=\sum \lambda^{\prime}(\theta)\left[1-\frac{z}{\lambda(\theta)}\right]
$$


where $\lambda^{\prime}(\theta)$ denotes the derivatives of the model function (4.12) over the parameters $\theta$, which has been given in part 2 .

Apparently, the above problem is a non-linear optimization problem, which requires an efficient and very stable algorithm for application. There are many non-linear optimization methods available, such as Newton's method, Quasi-Newton method, non-linear conjugate gradient method, or truth-region method [68]. Among the existing non-linear optimization methods, the Quasi-Newton method is the most efficient and stable algorithm, which has big advantages in applications.

\subsubsection{BFGS algorithm}

BFGS algorithm is a very important Quasi-Newton method [69], in which the Hessian matrix is approximated with the results of the pervious iteration. The estimation of the Hessian matrix can be done iteratively. The implantation of the BFGS algorithm can be described by as follows [70].

\section{Algorithm 4.2}

Initialize a starting point $x_{0}$, Hessian matrix $H_{0}$, and convergence tolerance $\epsilon>0$;

set $k=0 ; x=x_{0}$.

while $\left\|\nabla f_{k}\right\|>\epsilon$;

Calculate search direction

$$
p_{k}=-H_{k} \nabla f_{k}
$$

Determine the step size by,

$$
\alpha_{k}=\operatorname{linese\operatorname {arch}}\left(p_{k}\right)
$$

Update iterative variable

$$
x_{k+1}=x_{k}+\alpha_{k} p_{k}
$$

Calculate

$$
\begin{aligned}
& s_{k}=x_{k+1}-x_{k} ; \\
& y_{k}=\nabla f_{k+1}-\nabla f_{k} ; \\
& \rho_{k}=\frac{1}{y_{k}^{T} s_{k}} ;
\end{aligned}
$$

Update Hessian matrix

$$
\begin{aligned}
& H_{k+1}=\left(I-\rho_{k} s_{k} y_{k}^{T}\right) H_{k}\left(I-\rho_{k} s_{k} s_{k}^{T}\right)+\rho_{k} s_{k} s_{k}^{T} \\
& k=k+1
\end{aligned}
$$

\section{end (while)}

where $\|\cdot\|$ denotes the norm of a vector, $T$ denotes matric transposition, $\nabla f_{k}$ is the gradient of the objective function, $\alpha_{k}$ is the step size, and $p_{k}$ is the direction in the $k^{\text {th }}$ iteration.

A line search is required for implementing the BFGS algorithm, to guarantee global convergence of the fitting algorithm. Simulations show that a Back-tracking line search [71] technique is already good enough for the Gaussian fitting problem. The Back- 
tracking method is very widely used, easy to implement, and highly efficient.

\subsection{Simulation}

I have implemented Monte Carlo simulations to test the localization accuracy of the proposed algorithm. The simulation is written in Matlab and was run on a 64bit Windows 10 OS computer with i5-6600@3.3GHz processor and 32GB RAM.

Confocal light spots are simulated by a Gaussian function; Poisson noise is added to the simulated images; the localization error is calculated by $e=\sqrt{\Delta_{x}^{2}+\Delta_{y}^{2}}$. Finally, average and standard deviation of 2000 localizing results with the generated noisy data are determined.

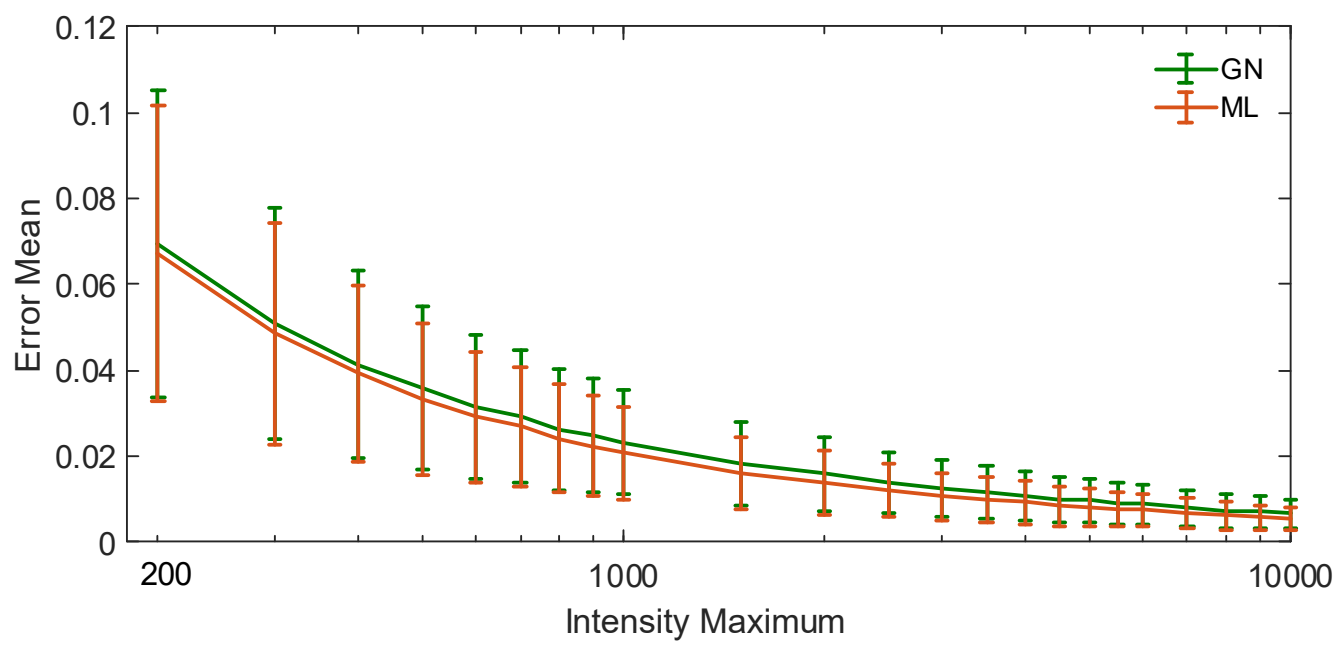

Figure 4.1 Average and standard deviation of localization error (pixels) vs. intensity maximum.

GN is Gauss-Newton algorithm, ML denotes Maximum Likelihood fitting method.

Figure 4.1 shows that the accuracy increases with increasing the intensity. The simulation shows that the MLE-based fit is more accurate than the Least-Square fit. However, the accuracy of both methods is quite close to each other over an intensity range of 200 to $10^{4}$. Because an intensity larger than 200 can be guaranteed in ISM reference measurements, the Least-Square method seems to be good enough for ISM localization application. Therefore, due to its efficiency, I selected the Least-Square based Gaussian fit algorithm as the localizer for the reconstruction software.

Table 4.1 Average CPU time (ms) for $10 \times 10$ image fitting

\begin{tabular}{|c|c|c|c|}
\hline Method & GN & ML & Fminsearch \\
\hline CPU Time & 1.588 & 2.590 & 6.931 \\
\hline
\end{tabular}

Table 4.1 shows that the proposed methods are much more efficient than existing Matlab solvers, which delivers completely the same solutions as the Gauss-Newton algorithm. It also shows that the Gauss-Newton algorithm is more efficient than the 
BFGS algorithm, which takes about $1 \mathrm{~ms}$ more. The reason is that the MLE is a more accurate model for fitting data with Poisson noise. This results in a higher precision; however, it is a more complex non-linear problem, which requires more computing time.

Besides the SNR, the size of the selected area used for fitting is also a factor that impacts the fitting accuracy. This is demonstrated in figure 4.2.

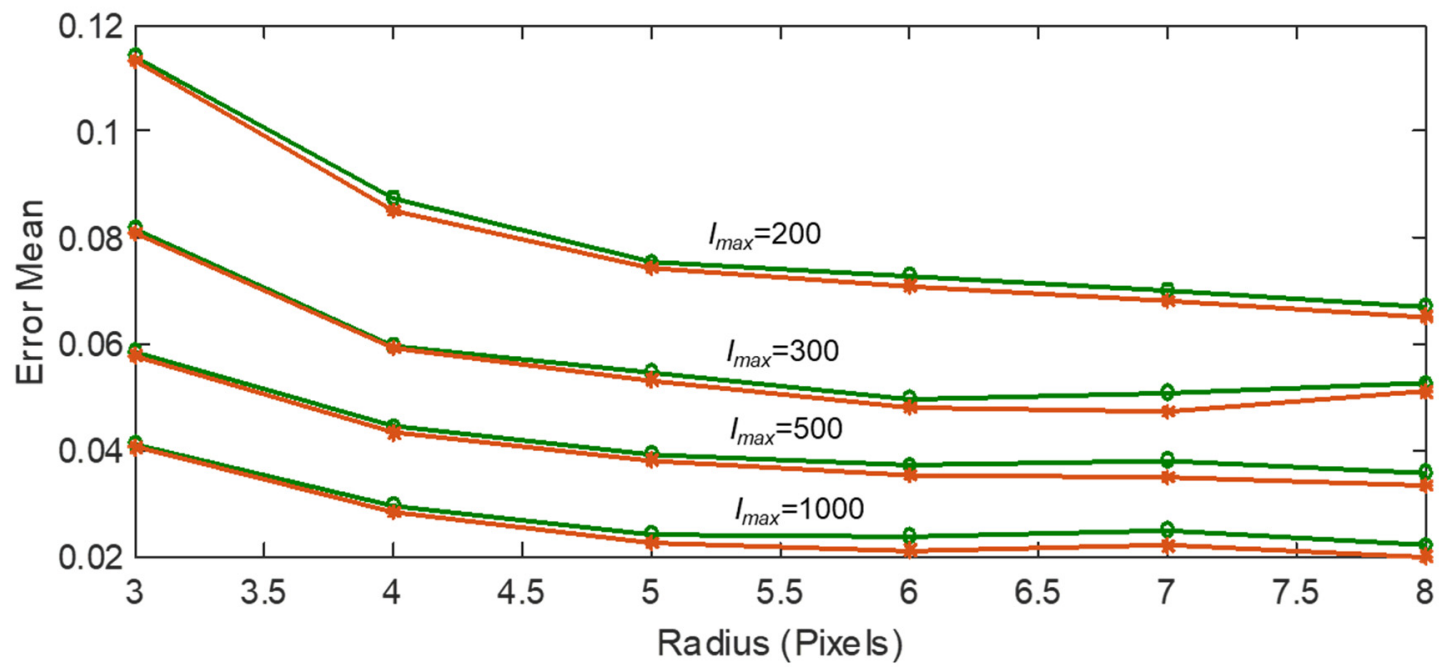

Figure 4.2 Error mean vs. radius of the selected area of the observed image with FWHM equal to 10 pixels.

The simulation shows that the fit accuracy of both techniques increases with increasing the area of the Gaussian function. To obtain a good fit accuracy, the size of the selected area should be large enough. Simulations show that one should chose an area size large enough so that the signal has fallen off to $1 / 20$ of its maximum. This guarantees a good fitting accuracy and efficiency, and is shown at Radius $=7$ in figure 4.2.

\subsection{Discussion}

In this chapter, two accurate and efficient fit algorithms were developed for 2D Gaussian fitting. First, a simplified fit model was built that decreases the complexity of the fit model and algorithm. The iteration formula for the fit algorithm was then analyzed based on the goal function and using a Gauss-Newton algorithm and a BFGS algorithm. Next, a simple but efficient algorithm was proposal for initial value estimation. Finally, simulations were implemented to test the accuracy of the developed algorithm. The result shows that the ML fit is more accurate than LSQ fit. However, LSQ has tha advantage of being more efficient. Based on the simulation results, I can conclude that a LSQ fit is more suitable for high SNR data fitting, while a ML fit is more suitable for lower SNR data containing Poisson noise. In addition, the size of the selected area used for fitting is also an important factor that affects the fit accuracy. 


\section{Chapter 5}

\section{Simulation of}

\section{Structured Illumination}

Microscopy

\subsection{Introduction}

Structured Illumination Microscopy (SIM) is a powerful technique to realize linear super-resolution, which is more and more widely used today to its relative simplicity, compatibility with conventional fluorophores, and high-quality image output. In applications, two dimensional SIM has achieved great success, and many good results have been reported since its first publication [72]. Moreover, three-dimensional variants of SIM are nowadays available [26, 27].

The principle of SIM is very simple: super-resolution is achieved by illuminating a sample with a structured, i.e. spatially modulated, illumination. Mathematically, spatially modulating the object function corresponds to convolving the object function with the modulation function in the Fourier domain, which results in an extension of the Fourier domain support. As the support of the Fourier spectrum of the object is extended by convolution, super-resolution is achieved. Technically, general SIM can be realized by using a spatial cosine function as the illumination pattern. The Fourier transform of a cosine function is a delta function. Convolution with it will shift the Fourier spectrum. In this way, higher frequencies of the object function along the axis of the modulation can be measured, without improving the optical power of the imaging system. Therefore, the main ingredients of SIM are the structured illumination and image reconstruction.

Reconstruction of a SIM image is a very common problem in fluorescence microcopy. Basically, a SIM image can be reconstructed by Fourier reconstruction, which separates the Fourier spectrum along each modulation direction from the measured raw images, and then recombines the calculated incomplete Fourier spectrum in the Fourier domain to form a complete Fourier spectrum of the object's image. There is open source software available for easy SIM reconstruction [73]. These techniques are have advantage in efficiency and robustness compared to iterative reconstruction methods. However, it is not so easy to implement them in practice. The problem of Fourier reconstruction is that the frequency and phase of modulation function have to be estimated accurately. However, it is very difficult to guarantee that due to noise and system alignment errors. Besides that, the final super-resolved image is reconstructed based on Wiener filtering, which requires knowledge of the exact power spectrum of the real image and the noise, 
so that one can calculate the signal-noise-ratio ratio which is required for a perfect image reconstruction. Usually, the power spectrum of the real image and noise is not available. Instead of estimating the exact power spectrum of the real image and noise, a damping parameter is used to approximate the signal-to-noise ratio. Therefore, it is challenging to restore a perfect image with Fourier reconstruction. Often, the results suffer from artifacts.

Deconvolution SIM is another promising method for SIM image reconstruction [74, 75]. Compared to Fourier reconstruction, in deconvolution SIM it is easier to deal with noise and artifacts. The advantage of deconvolution SIM is that one can introduce a regularization, for high quality image restoration. Because reconstruction is done in real space, any phase error, apodization threshold, or estimation of the Wiener filter regularization parameter are no crucial problems. Deconvolution SIM has been studied comprehensively in the literature [76].

In this chapter, the principle of SIM is illustrated by a mathematical analysis and simulation. Both Fourier reconstruction SIM and deconvolution SIM are implemented. In deconvolution SIM simulations, different regularization techniques are introduced for denoising and artifact removal. The results show that Fourier reconstruction SIM is faster, but results in numerous artifacts, while deconvolution SIM is easier to implement and achieves higher image quality with proper regularization.

\subsection{Fourier Reconstruction Based SIM}

Here, I consider a cosine modulation as an example, as shown in Figure 5.1.

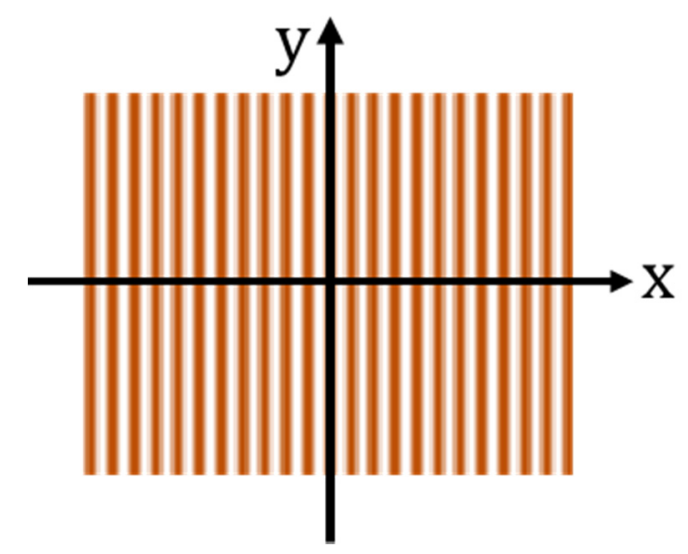

Figure 5.1

The modulation function can be written by

$$
I(\boldsymbol{r})=I_{0}+A \cos (2 \pi \boldsymbol{p} \cdot \boldsymbol{r}+\phi)
$$

where $A$ denotes the modulation amplitude, $I_{0}$ denotes the background, $m$ denotes the depth of modulation, $\boldsymbol{p}$ denotes the spatial frequency vector of the illumination function, $\boldsymbol{r}$ denotes the spatial coordinate in object space, and $\phi$ is the phase of the 
cosine modulation function.

According to linear imaging theory, the measured image is given by

$$
D(\boldsymbol{r})=[I(\boldsymbol{r}) \cdot S(\boldsymbol{r})] \otimes H(\boldsymbol{r})+N(\boldsymbol{r})
$$

Performing a Fourier transform on the above function, we have

$$
D(\boldsymbol{k})=[I(\boldsymbol{k}) \otimes S(\boldsymbol{k})] \cdot H(\boldsymbol{k})+N(\boldsymbol{k})
$$

It is more convenient to write the above function in matrix form as

$$
\begin{aligned}
& D(\boldsymbol{k})=[I(\boldsymbol{k}) \otimes S(\boldsymbol{k})] \cdot H(\boldsymbol{k})+N(\boldsymbol{k}) \\
& \left.=\left\{I_{0}+A \cdot \frac{1}{2}\left[\delta(\boldsymbol{k}-\boldsymbol{p}) e^{i \phi}+\delta(\boldsymbol{k}+\boldsymbol{p}) e^{-i \phi}\right] \otimes S(\boldsymbol{k})\right\} \cdot H(\boldsymbol{k})+N(\boldsymbol{k})\right] \\
& \left.=\left[I_{0} S(\boldsymbol{k})+\frac{A}{2} S(\boldsymbol{k}-\boldsymbol{p}) e^{i \phi}+\frac{A}{2} S(\boldsymbol{k}+\boldsymbol{p}) e^{-i \phi}\right] \cdot H(\boldsymbol{k})+N(\boldsymbol{k})\right] \\
& =\left[\begin{array}{lll}
I_{0} & \frac{A}{2} e^{i \phi} & \frac{A}{2} e^{-i \phi}
\end{array}\right]\left[\begin{array}{c}
S(\boldsymbol{k}) H(\boldsymbol{k}) \\
S(\boldsymbol{k}-\boldsymbol{p}) H(\boldsymbol{k}) \\
S(\boldsymbol{k}+\boldsymbol{p}) H(\boldsymbol{k})
\end{array}\right]+N(\boldsymbol{k})
\end{aligned}
$$

which shows that the cosine modulation results in a shift of the Fourier spectrum by $\pm \boldsymbol{p}$. In other words, higher frequency information can be obtained from the "structureilluminated" image. Obviously, a single $D(\boldsymbol{k})$ is not enough to solve the above equation to obtain the object function $S(\boldsymbol{k})$. To solve the above equation, more conditions are needed. Indeed, we can change the phase of the modulation function, which corresponds to shifting the modulation by a distance $\Delta r$ along the modulation direction, and measure more images. In theory, three images are required at minimum. Then, the equation can be written as

$$
\begin{aligned}
{\left[\begin{array}{l}
D_{1}(\boldsymbol{k}) \\
D_{2}(\boldsymbol{k}) \\
D_{3}(\boldsymbol{k})
\end{array}\right]=} & {\left[\begin{array}{lll}
I_{0} & \frac{A}{2} e^{i \phi_{1}} & \frac{A}{2} e^{-i \phi_{1}} \\
I_{0} & \frac{A}{2} e^{i \phi_{2}} & \frac{A}{2} e^{-i \phi_{2}} \\
I_{0} & \frac{A}{2} e^{i \phi_{3}} & \frac{A}{2} e^{-i \phi_{3}}
\end{array}\right]\left[\begin{array}{c}
S(\boldsymbol{k}) H(\boldsymbol{k}) \\
S(\boldsymbol{k}-\boldsymbol{p}) H(\boldsymbol{k}) \\
S(\boldsymbol{k}+\boldsymbol{p}) H(\boldsymbol{k})
\end{array}\right]+\left[\begin{array}{l}
N_{1}(\boldsymbol{k}) \\
N_{2}(\boldsymbol{k}) \\
N_{3}(\boldsymbol{k})
\end{array}\right](5.5) } \\
= & {\left[\begin{array}{lll}
1 & \frac{1}{2} \frac{A}{I_{0}} e^{i \phi_{1}} & \frac{1}{2} \frac{A}{I_{0}} e^{-i \phi_{1}} \\
1 & \frac{1}{2} \frac{A}{I_{0}} e^{i \phi_{2}} & \frac{1}{2} \frac{A}{I_{0}} e^{-i \phi_{2}} \\
1 & \frac{1}{2} \frac{A}{I_{0}} e^{i \phi_{3}} & \frac{1}{2} \frac{A}{I_{0}} e^{-i \phi_{3}}
\end{array}\right]\left[\begin{array}{c}
S(\boldsymbol{k}) H(\boldsymbol{k}) \\
S(\boldsymbol{k}-\boldsymbol{p}) H(\boldsymbol{k}) \\
S(\boldsymbol{k}+\boldsymbol{p}) H(\boldsymbol{k})
\end{array}\right]+\left[\begin{array}{c}
N_{1}(\boldsymbol{k}) \\
N_{2}(\boldsymbol{k}) \\
N_{3}(\boldsymbol{k})
\end{array}\right] }
\end{aligned}
$$

Let $\gamma=\frac{A}{I_{0}}$, which indicates the modulation depth, and $M=\left[\begin{array}{ccc}1 & \frac{\gamma}{2} e^{i \phi_{1}} & \frac{\gamma}{2} e^{-i \phi_{1}} \\ 1 & \frac{\gamma}{2} e^{i \phi_{2}} & \frac{\gamma}{2} e^{-i \phi_{2}} \\ 1 & \frac{\gamma}{2} e^{i \phi_{3}} & \frac{\gamma}{2} e^{-i \phi_{3}}\end{array}\right]$, 
which is invertible, then $\left[\begin{array}{c}S(\boldsymbol{k}) H(\boldsymbol{k}) \\ S(\boldsymbol{k}-\boldsymbol{p}) H(\boldsymbol{k}) \\ S(\boldsymbol{k}+\boldsymbol{p}) H(\boldsymbol{k})\end{array}\right]$ can be estimated by
\[ \left[\begin{array}{c}C(\boldsymbol{k}) \\ C(\boldsymbol{k}-\boldsymbol{p}) \\ C(\boldsymbol{k}+\boldsymbol{p})\end{array}\right]=\left[\begin{array}{c}S(\boldsymbol{k}) H(\boldsymbol{k}) \\ S(\boldsymbol{k}-\boldsymbol{p}) H(\boldsymbol{k}) \\ S(\boldsymbol{k}+\boldsymbol{p}) H(\boldsymbol{k})\end{array}\right]=M^{-1}\left[\begin{array}{l}D_{1}(\boldsymbol{k}) \\ D_{2}(\boldsymbol{k}) \\ D_{3}(\boldsymbol{k})\end{array}\right] \]

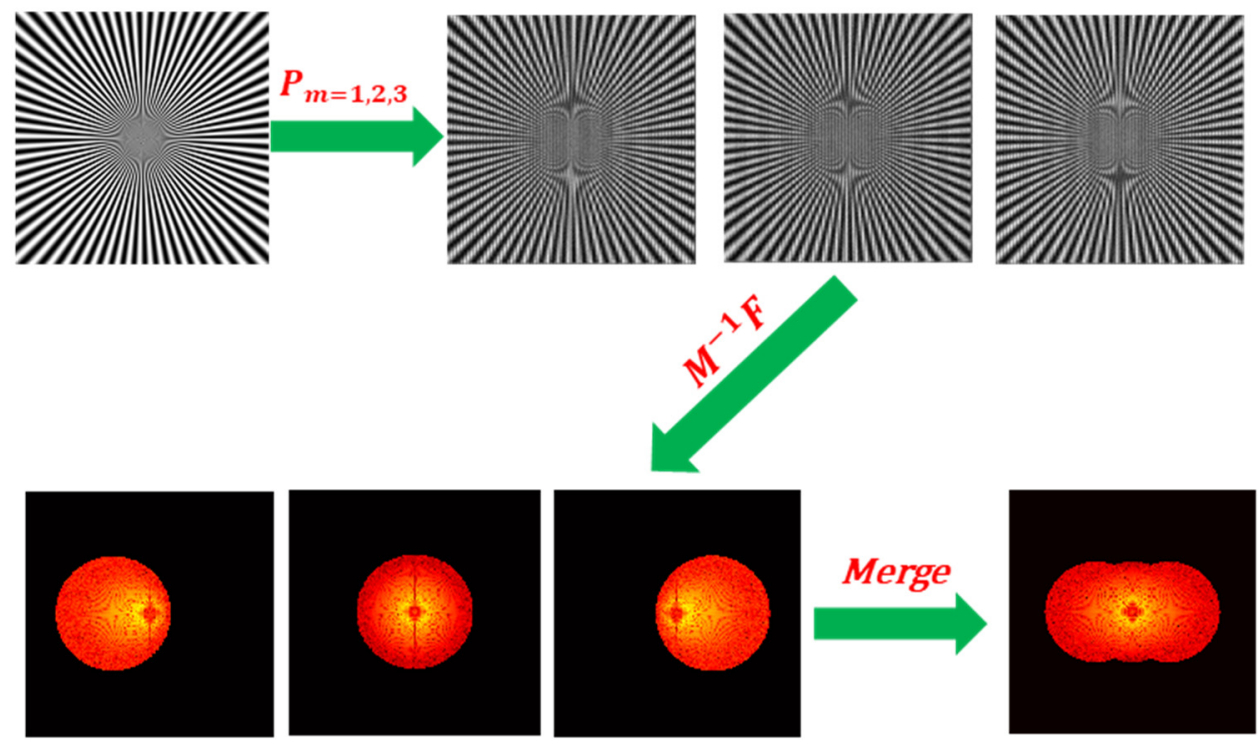

Figure 5.2

In order to obtain enough spectra to fill the Fourier domain completely, more images are measured by changing the angle of the modulation direction. This corresponds to a rotation of the mask. For example, we can measure three groups of triple-images along the modulation directions $0^{\circ}, 60^{\circ}$, and $120^{\circ}$, respectively. In practice, nine images are enough to reconstruct a final SIM image. The index of the images is defined by $m=[1,2,3,4,5,6,7,8,9]$; finally, we obtain a sufficiently sampled Fourier spectrum to reconstruct a super-resolved SIM image by

(I) in angle $\theta=0$ :

$$
\left[\begin{array}{c}
C_{1}(\boldsymbol{k}) \\
C_{2}(\boldsymbol{k}-\boldsymbol{p}) \\
C_{3}(\boldsymbol{k}+\boldsymbol{p})
\end{array}\right]_{0}=\left[\begin{array}{c}
S(\boldsymbol{k}) H(\boldsymbol{k}) \\
S(\boldsymbol{k}-\boldsymbol{p}) H(\boldsymbol{k}) \\
S(\boldsymbol{k}+\boldsymbol{p}) H(\boldsymbol{k})
\end{array}\right]_{0}=M^{-1}\left[\begin{array}{c}
D_{1}(\boldsymbol{k}) \\
D_{2}(\boldsymbol{k}) \\
D_{3}(\boldsymbol{k})
\end{array}\right]
$$

(II) in angle $\theta=\frac{\pi}{3}$ :

$$
\left[\begin{array}{c}
C_{4}(\boldsymbol{k}) \\
C_{5}(\boldsymbol{k}-\boldsymbol{p}) \\
C_{6}(\boldsymbol{k}+\boldsymbol{p})
\end{array}\right]_{\frac{\pi}{3}}=\left[\begin{array}{c}
S(\boldsymbol{k}) H(\boldsymbol{k}) \\
S(\boldsymbol{k}-\boldsymbol{p}) H(\boldsymbol{k}) \\
S(\boldsymbol{k}+\boldsymbol{p}) H(\boldsymbol{k})
\end{array}\right]_{\frac{\pi}{3}}=M^{-1}\left[\begin{array}{c}
D_{4}(\boldsymbol{k}) \\
D_{5}(\boldsymbol{k}) \\
D_{6}(\boldsymbol{k})
\end{array}\right]
$$


(III) in angle of $\theta=\frac{2 \pi}{3}$ :

$$
\left[\begin{array}{c}
C_{7}(\boldsymbol{k}) \\
C_{8}(\boldsymbol{k}-\boldsymbol{p}) \\
C_{9}(\boldsymbol{k}+\boldsymbol{p})
\end{array}\right]_{\frac{2 \pi}{3}}=\left[\begin{array}{c}
S(\boldsymbol{k}) H(\boldsymbol{k}) \\
S(\boldsymbol{k}-\boldsymbol{p}) H(\boldsymbol{k}) \\
S(\boldsymbol{k}+\boldsymbol{p}) H(\boldsymbol{k})
\end{array}\right]_{\frac{2 \pi}{3}}=M^{-1}\left[\begin{array}{c}
D_{7}(\boldsymbol{k}) \\
D_{8}(\boldsymbol{k}) \\
D_{9}(\boldsymbol{k})
\end{array}\right]
$$

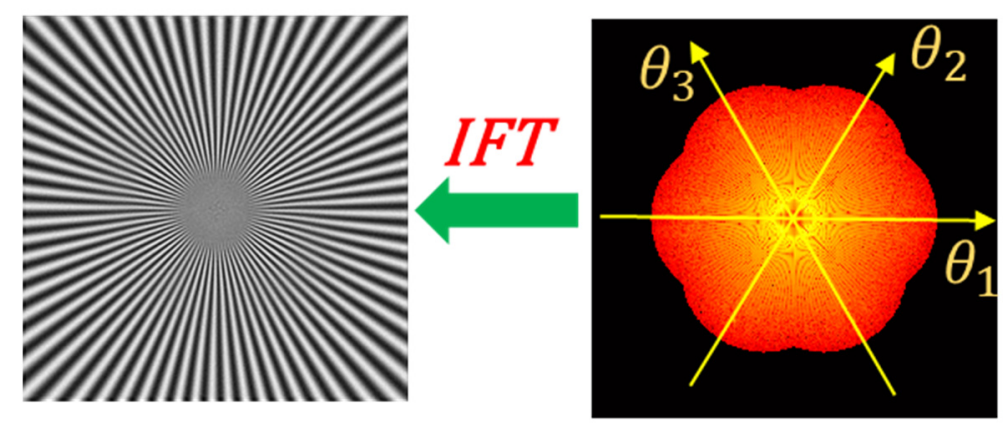

Figure 5.3

The final super-resolved image is obtained by merging all components to $S(\boldsymbol{k})$

$=\frac{\sum_{m}\left[H^{*}(\boldsymbol{k}) \cdot C_{1+3 m}(\boldsymbol{k})+H^{*}(\boldsymbol{k}+\boldsymbol{p}) \cdot C_{2+3 m}(\boldsymbol{k}+\boldsymbol{p})+H^{*}(\boldsymbol{k}-\boldsymbol{p}) \cdot C_{3+3 m}(\boldsymbol{k}-\boldsymbol{p})\right]_{\frac{\boldsymbol{m} \boldsymbol{\pi}}{3}}}{\omega^{2}+\sum_{m}\left[H^{2}(\boldsymbol{k})+H^{2}(\boldsymbol{k}+\boldsymbol{p})+H^{2}(\boldsymbol{k}-\boldsymbol{p})\right]_{\frac{m \pi}{3}}}$

where $m=[0,1,2]$.

\subsubsection{Calculate Frequency and Phase of the Modulation Pat- tern}

The above analysis shows that the transformation matrix $M$ is the key for image reconstruction in SIM. The accuracy of estimated frequency and phase values will affect the quality of the final super-resolved image. In order to guarantee high accuracy, the estimation is realized by fitting a reference image obtained from a homogenous sample that is excited with a cosine intensity pattern.

Assume $\theta=\left(A, p, \phi, \alpha, I_{0}\right)$, given the measured reference image of the pattern, the PSF of the imaging system and coordinate $r$, the cost function for fitting can be written as

$$
E(\theta)=\left\|P S F *\left[I_{0}+A \cos (2 \pi \boldsymbol{p} \cdot \boldsymbol{r}(\alpha)+\phi)\right]-\mathrm{y}\right\|_{2}^{2}
$$

where $\boldsymbol{r}(\alpha)$ is the coordinate rotated by angle $\alpha$. The above problem can be solved by existing solvers, such as the fminsearch function of Matlab. Usually, strongly nonlinear optimization problems require good initial values to guarantee that the optimization algorithm converges to the correct solution. Therefore, it is necessary to estimate a proper initial value of $\theta$ in practice. Actually, the amplitude $A$ and background $I_{0}$ are very easy to estimate by simply taking the peak value and the valley value of the measured pattern, while the frequency $p$ and the rotation angle $\alpha$ can be estimated through 
the position of the two conjugated peaks of the Fourier spectrum of the measured image. The phase $\phi$ can be simply set to an arbitrary value within the interval $[0,2 \pi]$.

\subsubsection{Frequency Shifting}

The Fourier separated spectrum $C_{m}\left(\boldsymbol{k}-\boldsymbol{p}_{\boldsymbol{m}}\right)$ has to be shifted by $\boldsymbol{p}_{\boldsymbol{m}}$ to form the correct spectrum of the final super-resolved image. Basically, it can be done efficiently by taking advantage of the Fourier shifting property, which is described by

$$
\mathrm{F}\left(\omega-\omega^{\prime}\right) \leftrightarrow f(r) \cdot e^{j \omega^{\prime} r}
$$

where $F(\omega)$ is the Fourier transform of $f(r)$.

\subsubsection{Simulation}

In order to test the SIM reconstruction algorithm, I use the same object image as presented in Ref. [77] and write an algorithm based on the above analysis to simulate the SIM imaging process. The object image is shown in Figure 5.4.
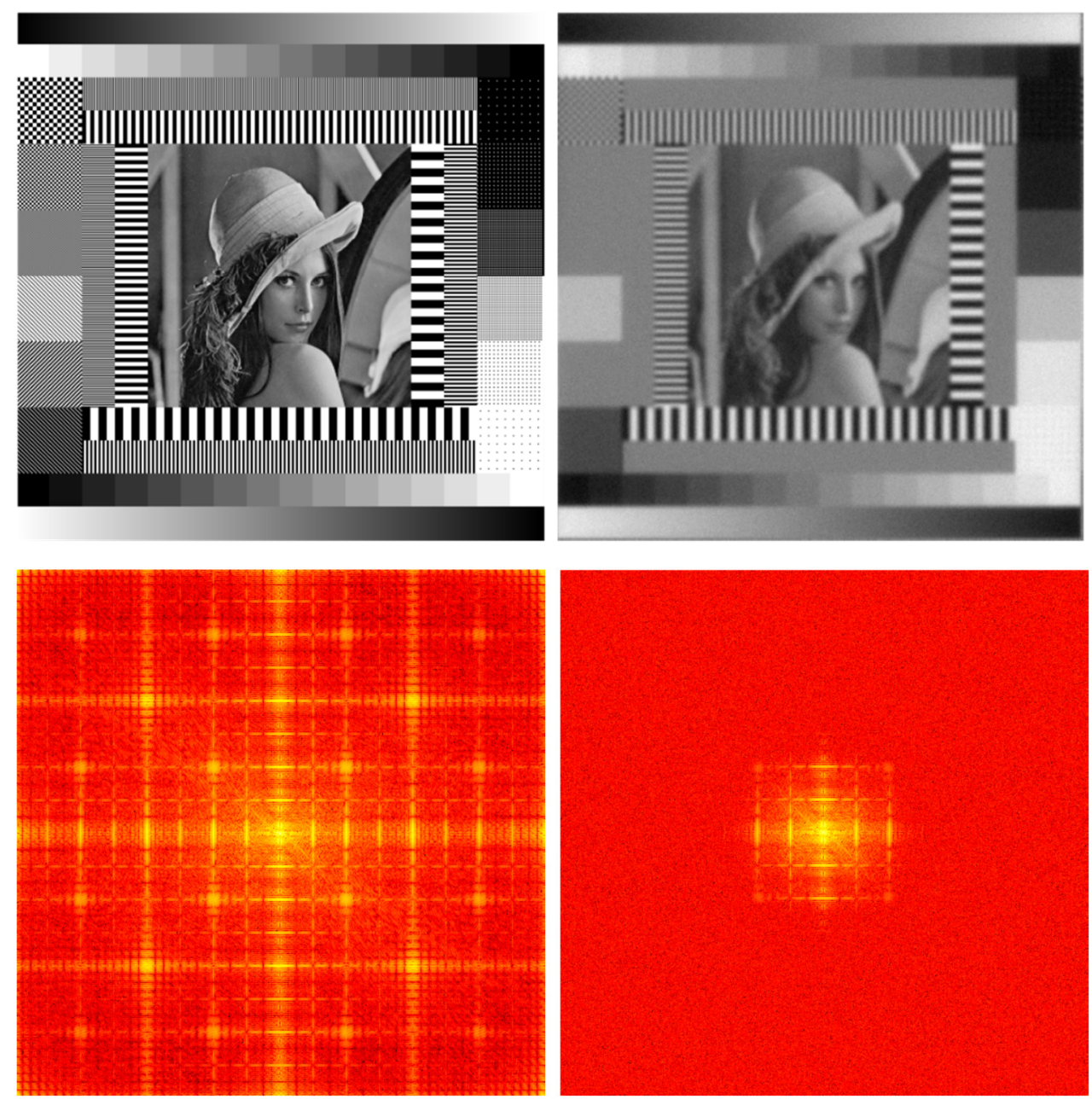

Figure 5.4 Left: ground-truth. Right: wide-field image. The bottom shows the Fourier spectra of the top images. 
The object image contains a large amount of high frequency components, which can be used to judge the reconstruction performance visually. The wide-field image is so blurred that many details with high spatial frequencies are invisible. Gaussian noise is added to the simulated raw images. The SNR of the raw image is $20 \mathrm{~dB}$.
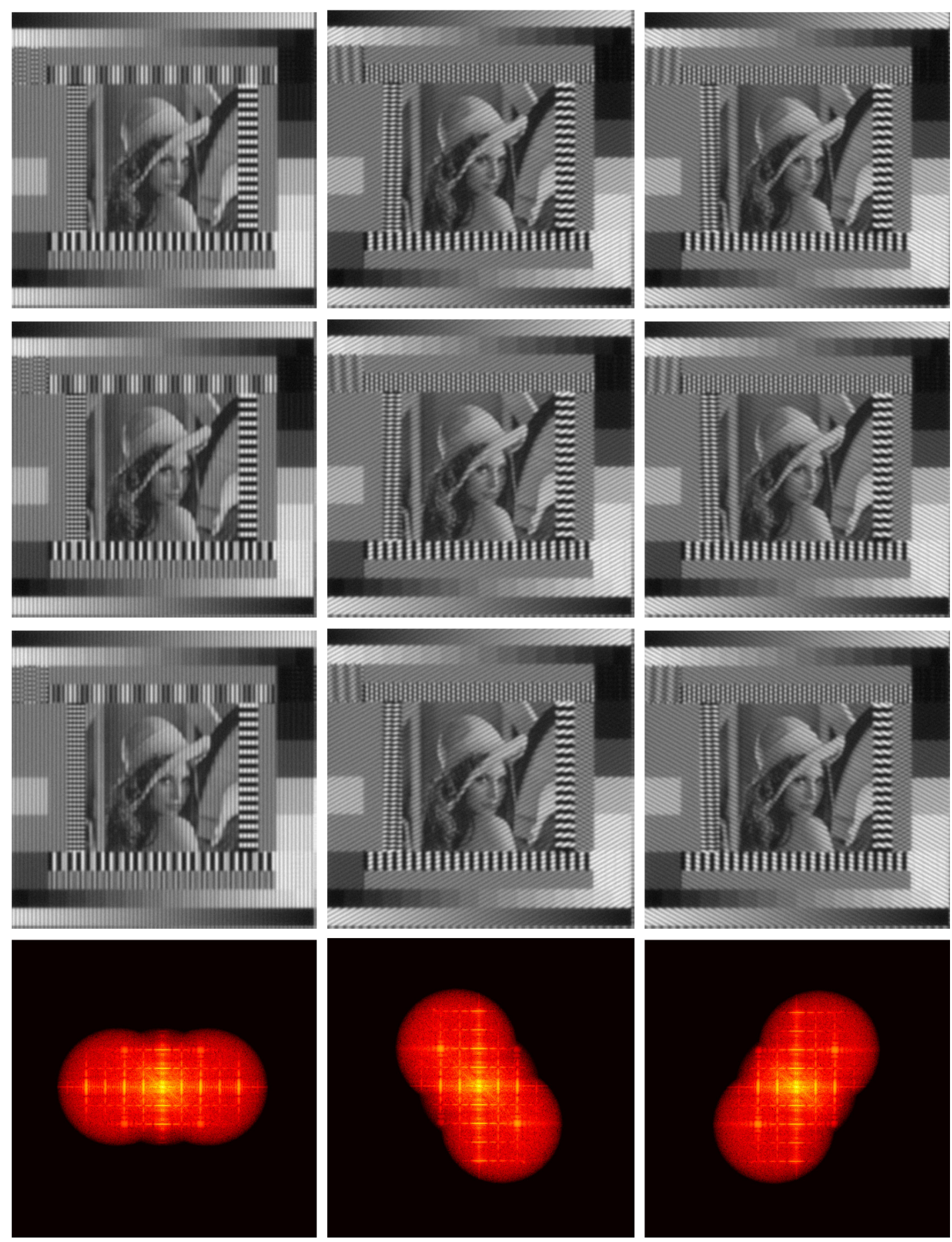

Figure 5.5 Simulated SIM raw images and the separated Fourier spectra. Along columns, the phase is changed, and along each row, the modulation angle

$$
\text { is changed }\left(\theta=0, \frac{\pi}{3}, \frac{2 \pi}{3}\right) \text {. }
$$

As the reconstruction is done in Fourier domain, the final image is obtained by an 
inverse Fourier transform of the reconstructed Fourier image data. The simulation shows that the separated Fourier spectrum has twice-increased support along the modulation direction than that of the wide-field image along. The whole spectrum reconstruction is shown in Figure 5.6 (Left). The extended support results in a significant resolution enhancement.
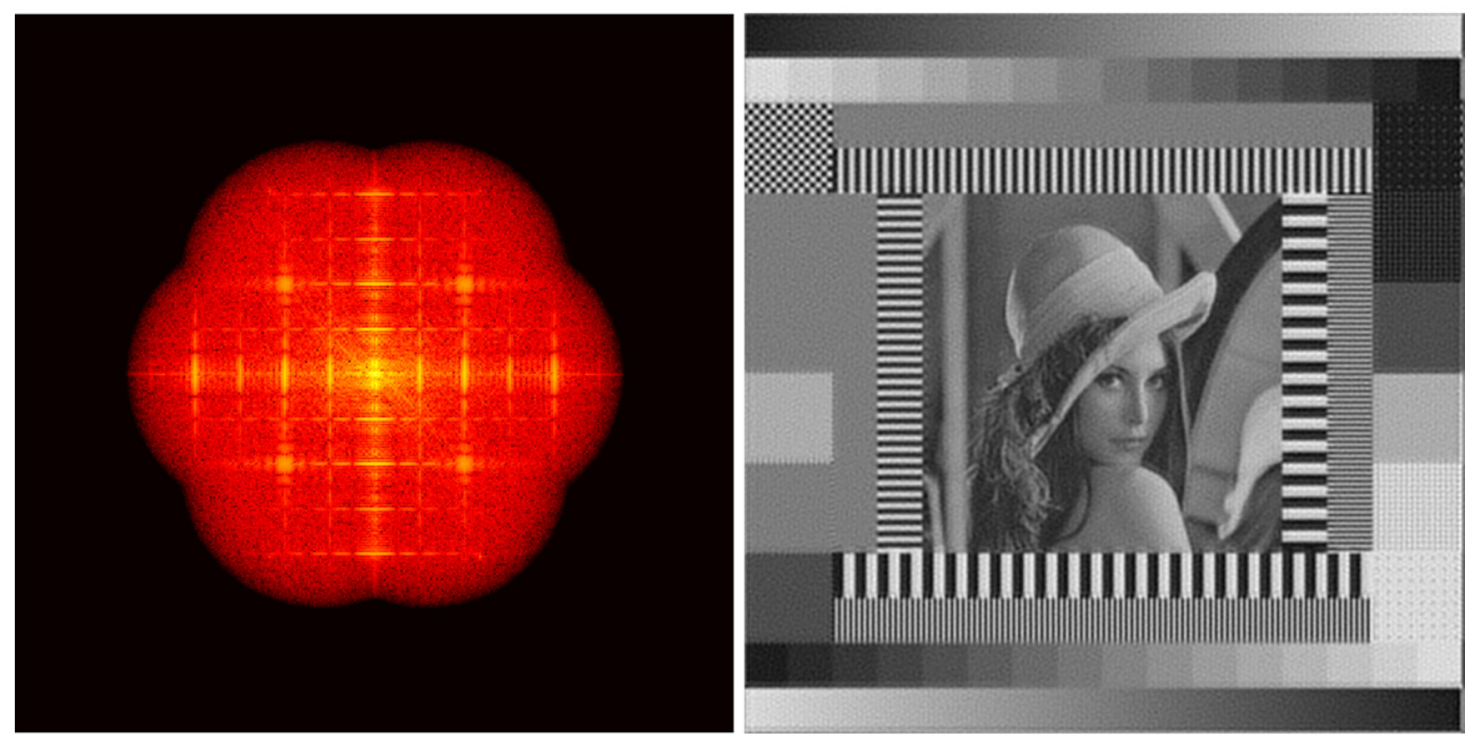

Figure 5.6 Reconstructed SIM image. Left: the reconstructed Fourier spectrum. Right: the final reconstructed super-resolution image.

It is very convenient to implement the SIM image recontruction in the Fourier domain. However, the reconstructed images often suffer from artifacts. This happens because the estimation of the modulation frequency and the phase are affected by noise. Moreover, there can be a phase difference between images reconstructed along differenct modulation directions. Due to all these errors, it is hard to gurantee that all components are perfectly merged. In contrast, reconstruction in real space can avoid these problems.

\subsection{Deconvolution SIM}

\subsubsection{Theory of Deconvolution SIM}

Deconvolution-based SIM reconstructs a SIM image in the spatial domain. Here it is easier to deal with noise and artifacts, as one can introduce a regularization. However, an accurate estimation of the modulation function is still required to guarantee a high quality of image reconstruction.

Mathematically, the measured image is given by

$$
I_{m}=H *\left(P_{m} \cdot f\right)
$$

where $f$ is the object function, $H$ is the PSF of the imaging system, and $P_{m}$ is the 
illumination function. There are multiple images with different modulation phases measured along different modulation directions. Taking advantage of all measured images, the cost function can be written as

$$
E(f)=\sum_{m=1}^{M} \frac{1}{2}\left\|H *\left(P_{m} \cdot f\right)-G_{m}\right\|^{2}
$$

whose gradient can be easily obtained by

$$
\frac{\partial E}{\partial f}=\sum_{m=1}^{M} H^{T} *\left[H *\left(P_{m} \cdot f\right)-G_{m}\right] \cdot P_{m}
$$

In practice, it is useful to introduce a regularization for image denoising and artifact removal. The cost function with TV regularization can be written as

$$
E(f)=\sum_{m=1}^{M} \frac{1}{2}\left\|H *\left(P_{m} \cdot f\right)-G_{m}\right\|^{2}+\lambda R(f)
$$

The corresponding derivative is then easily obtained as

$$
\frac{\partial E}{\partial f}=\sum_{m=1}^{M} H^{T} *\left[H *\left(P_{m} \cdot f\right)-G_{m}\right] \cdot P_{m}+\lambda \frac{\partial R(f)}{\partial f}
$$

The details of regularization and the derivative of the regularization function will be studied in the next chapter. A non-negative constraint is a very useful constraint for deconvolution. In fact, we can employ a simple transform $f=f^{2}$ to introduce a nonnegative constraint. The constrained cost function reads

$$
E(f)=\sum_{m=1}^{M} \frac{1}{2}\left\|H *\left(P_{m} \cdot f^{2}\right)-G_{m}\right\|^{2}+R\left(f^{2}\right)
$$

The corresponding derivative is easily obtained as

$$
\frac{\partial E}{\partial f}=2 f \cdot \sum_{m=1}^{M} H^{T} *\left[H *\left(P_{m} \cdot f^{2}\right)-G_{m}\right] \cdot P_{m}+2 \lambda f \cdot \frac{\partial R\left(f^{2}\right)}{\partial f}
$$

With this gradient analysis, the cost function is very easy to solve by using any optimization method, such as a non-linear conjugate gradient method, or a limited-memory BFGS algorithm, etc. Efficient algorithms for deconvolution with some important regularization techniques will be studied in the next chapter.

From (5.18), we can see that Deconvolution SIM requires knowledge of the exact PSF of the imaging system and the modulation function for modeling the measured image. This is perhaps the biggest challenge in practice. For simple modulation patterns like the cosine function, it is very easy to estimate the parameters of the modulation function by fitting. However, for more complex modulation patterns is not easy to calculate the modulation function without knowing the mathematical model. In such cases, an experimental estimation is required. Just as discussed above, the modulation function can be estimated based on measured reference images.

\subsubsection{Verification of Deconvolution SIM}

I verify Deconvolution SIM with simulations based on the same data set as used above. Total Variation regularization, Hessian regularization, and Roughness regularization are employed to remove noise and artifacts. The results are then compared to existing reconstruction methods, as shown in Figure 5.7. 

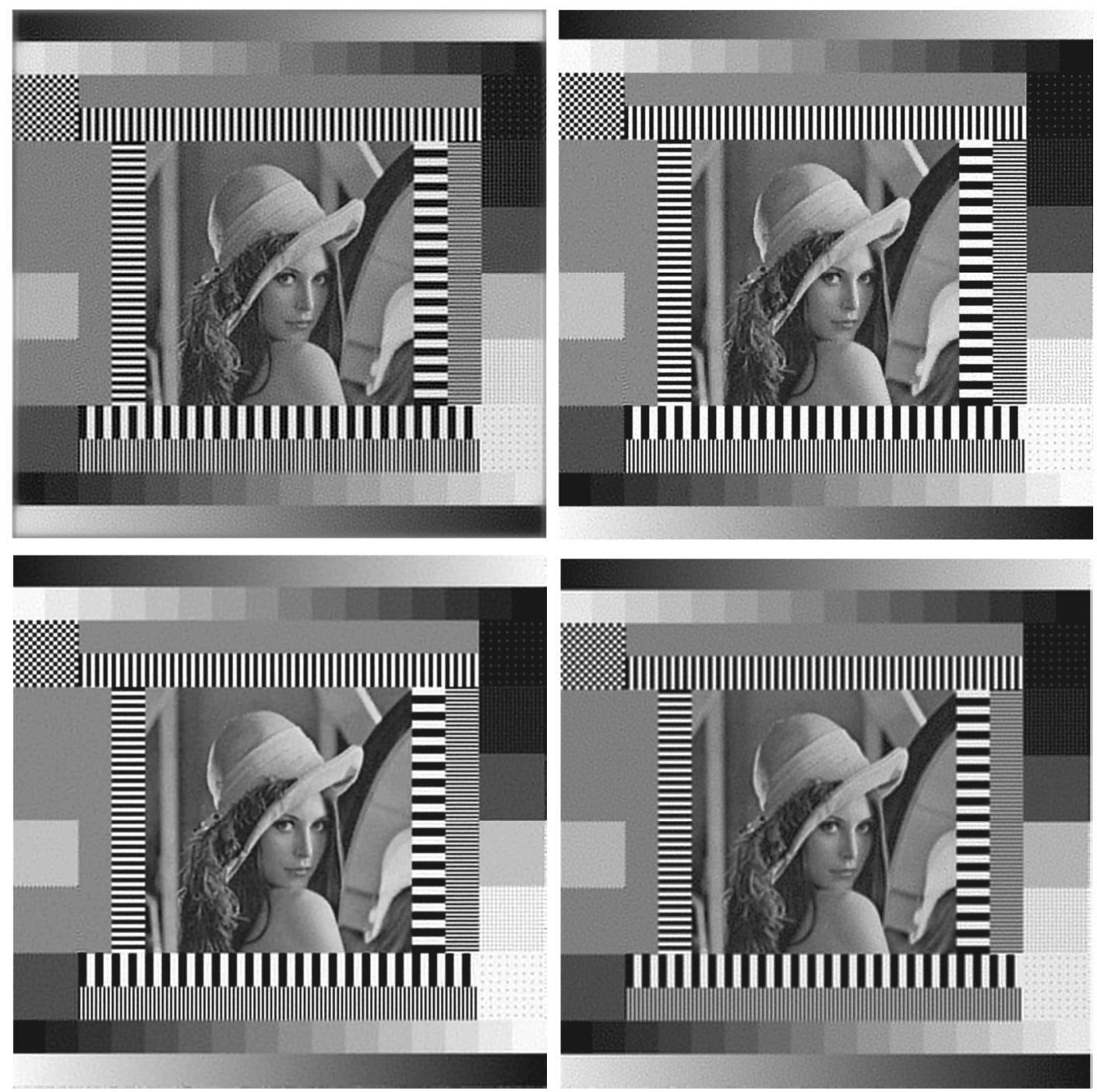

Figure 5.7 Deconvolution SIM. Top-left: Wiener filter without regularization. Top-right: TV regularization. Bottom-left: Hessian regularization. Bottom-right: Roughness regularization

The results show that Deconvolution SIM reconstruction methods outperform Fourier reconstruction. Noise and artifacts removal is significant. In contrast, TV regularization results in very sharp edges and high contrast, although slight artifacts are still visible. Hessian regularization and roughness regularization result in very smooth images; both suppress noise and artifacts very well. Visually, Hessian regularization seems to have the best performance, because the structure of the object image matches the property of Hessian regularization. In the next chapter, I will show that the TV regularization is more suitable for staircasing structures, Hessian regularization is more suitable for staircasing or linear structures, and roughness regularization is suitable to smooth and continuous structures. All these properties can be seen in the reconstructed images shown in Figure 5.7. Therefore, I believe that Deconvolution SIM is a very flexible technique for SIM image reconstruction, and that it is very promising for application. 


\subsection{Discussion}

In this chapter, I illustrated the principle of SIM via a mathematical analysis and simulation. I implemented a Fourier SIM reconstruction algorithm and deconvolution SIM reconstruction algorithms, and compared their results. The simulation results show that Fourier SIM is very efficient, but usually suffers from artifacts due to noise. In contrast, Deconvolution SIM is very flexible and works best for denoising and artifact removal, by using an appropriate regularization. 


\section{Chapter $6 \quad$ Solving L1-Norm Regularized Problems Based on Non-linear Optimization}

\subsection{Introduction}

$L_{1}$-norm optimization problems become more and more important nowadays, and they are widely used in many applications: signal reconstruction in compressed sensing, network training, signal or image denoising and deconvolution, etc. All these advanced techniques are based on $l_{1}$-norm optimization. Thus, efficient and accurate $l_{1}$-norm optimization methods are key for the development of more reliable, robust, and advanced information processing algorithms. So far, many algorithms and solvers have been developed to solve $l_{1}$-norm problems.

$L_{1}$-Magic is one of most well-known Matlab packages dedicated to solve any kind of compressed sensing (CS) reconstruction problems [78]. It was developed by Emmanuel Candes and Justin Romberg based on their early important work on compressed sensing [79]. All methods published in their article are mainly based on linear programming methods, which show a very good performance for CS reconstruction. However, these algorithms are not efficient when noise is involved, which is a very important issue in many applications.

Split-Bergman $[80,81]$ is another well-known method, which introduces external variables so that the problem can be transformed to some easy-to-solve sub-problems, that are then solved very efficiently with traditional optimization methods. The problem is that one has to implement the algorithm based on the problem's model, which can be challenging. The number of algorithms available for specific applications is still very limited so far: only TV denoising has been demonstrated to have a good performance. Simulations show that it is very sensitive to noise when applied to CS reconstruction problems, which makes the algorithm not computationally robust and fail-safe. Another problem is that more parameters are introduced, and multidimensional parameter tuning is difficult for many applications.

Another method that is based on Majorization Minimization (MM) [82] is also a very interesting method to solve $l_{1}$-norm optimization problems. The idea of MM is very similar to truth-region optimization: it introduces a convex function that is forced to satisfy some conditions to fit the objective function. Iteratively minimizing the sub- 
problem, e.g. the introduced convex function, will finally give the approximated solution of the objective function. These methods show a very good performance in data denoising and image restoration. However, it has the drawbacks that the form of calculation formula is not general to different type of 11-norm regularized problems and the analysis of the formula for calculation for specified application is very complicated. There is no solver available and there is still no constrained optimization version so far.

In contrast, it seems that little attention has been paid to non-linear optimization methods as the $l_{1}$-norm function is not smooth, which means that an analytical expression for the gradient is not available. In addition, the efficiency of numerically calculating the gradient can become a big issue when addressing large-scale optimization problems. However, to some extent one can circumvent these drawbacks. Some publications have reported about modified memory-limited Quasi-Newton methods. An Orthant-Wise limited-memory quasi-Newton (OWL-QN) method has been proposed [83], which extends the well-known non-linear algorithm, LBFGS, to non-smooth optimization. This method, however, is not flexible for solving a large variety of problems that do not take the $l_{1}$-norm of the variable itself, but its gradient or its transformation in another domain, take total variance regularization and wavelet transformation for example. This is quite common in practice when the observed signal itself is not sparse but localized in some domains, which requires regularization based on $l_{1}$-norm of some transformation of the signal to be reconstructed.

In this chapter, I show how to use a smoothing function to approximate the $l_{1}$-norm function to guarantee that the approximated function is everywhere differentiable. Then, the object function can be minimized via existing solvers, such as BFGS, LBFGS, LBFGS-B, or a non-linear conjugate gradient method, which have been developed for many years with very high performances. These mature methods can guarantee a robust, efficient, and accurate calculation. More importantly, many of these methods have been implemented and integrated on popular platforms such as Matlab or Python. Taking advantage of the smoothing transform and non-linear optimization solvers, $l_{1}$-norm optimization problems become much easier to deal with, so that those who are faced with $l_{1}$-norm optimization problems can focus on their actual problems without suffering from the implementation of specific optimization algorithms, or looking for dedicated solvers, which can be a big challenge and can take a lot of time.

\section{2 $\mathrm{L}_{1}$-norm Approximation by Smoothening function}

The function of the $l_{1}$-norm is not differentiable. However, it is possible to approximate it by some smooth function that can guarantee that the objective function is smooth everywhere while the output of each element term in the $l_{1}$-norm function is positivevalued.

$$
R(s)=\|x\|_{1} \cong \sum_{i} S\left(x_{i}\right)
$$


Ref [81] shows that $\|x\|_{1}$ can be used for generating a sparse solution. In order to better fit $\|x\|_{1}$, a function decaying from origin to $-\infty$ and $+\infty$ is more suitable than a constant. Indeed, there are many functions being positive and have a decaying property, for example, the well-known Gaussian function

with gradient

$$
S(x)=\sqrt{x^{2}+A e^{-\frac{x^{2}}{2 \sigma^{2}}}}
$$

$$
S^{\prime}(x)=\frac{x\left(1-\frac{A}{2 \sigma^{2}} e^{-\frac{x^{2}}{2 \sigma^{2}}}\right)}{\sqrt{x^{2}+A e^{-\frac{x^{2}}{2 \sigma^{2}}}}}
$$

and the reciprocal of square function

$$
S(x)=\left(x^{2}+\frac{\alpha}{x^{2}+1}\right)^{\frac{1}{2}}=\sqrt{x^{2}+\frac{\alpha}{x^{2}+1}}
$$

with gradient

$$
S^{\prime}(x)=\frac{x-\frac{\alpha x}{\left(x^{2}+1\right)^{2}}}{\sqrt{x^{2}+\frac{\alpha}{x^{2}+1}}}
$$

The parameters contained in both functions can be used to control the fit accuracy. Obviously, any precision of fitting can be achieved. The most important point is that the derivative is quite simple to calculate, which is crucial for applying non-linear optimization solvers. It is very easy to expand it to $l_{p}$-norm functions

$$
\begin{gathered}
S_{p}(x)=[S(x)]^{p} \\
S_{p}^{\prime}(x)=p S^{\prime}(x)[S(x)]^{p-1}
\end{gathered}
$$

The advantage of this conversion is that the smoothened $l_{1}$-norm penalty is not only applicable to the objective function, e.g. $\|x\|_{1}$, but also to any other form of $l_{1}$-norm penalty, such as Total-Variance regularization.

\subsection{Compressed Sensing Reconstruction Problem}

Compressed sensing (CS) is a novel technique for sparse signal processing [84, 85]. The theory of CS shows that a sparse signal can be perfectly reconstructed with a very small set of randomly measured data, which allows sampling rates lower than the Nyquist sampling limit. CS is very attractive for sampling rate and measurement time sensitive situations, such as fast MRI or CT. CS decreases the complexity of a measurement. However, it increases the complexity of reconstruction. Efficient reconstruction is the key for any CS application. In this section, I show how to solve efficiently basic CS reconstruction problems with the non-linear optimization. 


\subsubsection{Basis Pursuit (BP) Problem}

The goal function of the $B P$ problem can be represented as

$$
E(x)=\frac{1}{2}\|\Psi x-g\|_{2}^{2}+\|x\|_{1}
$$

Substituting $\|x\|_{1}$ with $S\left(x_{i}\right)$, the approximated goal function can be written as

$$
E(x)=\frac{1}{2}\|\Psi x-g\|_{2}^{2}+\sum_{i} S\left(x_{i}\right)
$$

and the corresponding gradient function is

$$
\frac{\partial E}{\partial x}=\Psi^{T}(\Psi x-g)+\sum_{i} S^{\prime}\left(x_{i}\right)
$$

where $\Psi$ is the measured data matrix, $g$ is the measured data, $x$ is the object to be reconstructed, and $T$ denotes matrix transposition. Since the goal function is smooth and an analytic expression for the gradient is available, it can be solved by any available non-linear optimization solver.

\subsubsection{Fourier Measured Compressed Sensing Reconstruc- tion Problem}

Fourier measured problems are very common in medical imaging, which samples in the Fourier domain and then reconstructs the image from the measured Fourier spectrum. In practice, it does not completely sample over the whole domain, but with a sampling mask. This requires that reconstruction algorithms are able to reconstruct an image from very limited sampling points. According to CS theory, a sparse signal in the form of a randomly measured matrix that satisfies the Restricted Isometry Property can be perfectly reconstructed, even with a sampling rate lower than required by Nyquist sampling theory. Many natural signals, such as sound, or biological images, are sparse, which makes CS very attractive for signal processing in these applications. In particular, TV-sparse is very common in the field of medical imaging. Therefore, TV sparse regularization can be used for CS reconstruction, which can be written as

$$
E(f)=\frac{1}{2}\|M F(f)-g\|_{2}^{2}+\lambda\|\nabla f\|_{1}
$$

The smoothened goal function is

$$
E(f)=\frac{1}{2}[M F(f)-g][M F(f)-g]^{*}+\lambda \iint S\left(\nabla_{x} f\right)+S\left(\nabla_{y} f\right) d x d y
$$

where $M$ is the random measurement matrix, $F$ denotes Fourier transform, $*$ denotes complex conjugation, $\nabla$ is the gradient operator, and $\lambda$ is a Lagrange multiplier. In matrix form, the gradient function can be found to be

$$
\begin{aligned}
\frac{\partial E}{\partial f}= & \frac{1}{2} \frac{\partial[M F f-g]}{\partial f}[M F f-g]^{*}+\frac{1}{2} \frac{\partial[M F f-g]^{*}}{\partial f}[M F f-g] \\
& \quad-\lambda\left[\nabla_{x} S^{\prime}\left(\nabla_{x} f\right)+\nabla_{y} S^{\prime}\left(\nabla_{y} f\right)\right] \\
= & \frac{1}{2} F[M F f-g]^{*}+\frac{1}{2} F^{*}[M F f-g]-\lambda\left[\nabla_{x} S^{\prime}\left(\nabla_{x} f\right)+\nabla_{y} S^{\prime}\left(\nabla_{y} f\right)\right]
\end{aligned}
$$


where $f$ denotes a vector, $F$ is the Discrete Fourier transform (DFT) matrix, $\nabla_{x}, \nabla_{y}$ are discrete gradient operators along the $x, y$ directions, respectively, which corresponds to the diff function in Matlab and has the same meaning as the operators in the following TV definition. The main operations in the above formula involve only Fourier transforms and difference calculations, which means it is very easy to implement on a computer. In the case of introducing non-negative constraint, it is very easy to convert a constrained optimization problem to an unconstrained optimization problem by substituting $f$ by $f^{2}$. The objective function then becomes

$$
E(f)=\frac{1}{2}\left[M F\left(f^{2}\right)-g\right]\left[M F\left(f^{2}\right)-g\right]^{*}+\lambda \iint S\left(\nabla_{x} f^{2}\right)+S\left(\nabla_{y} f^{2}\right) d x d y
$$

The corresponding gradient is

$$
\begin{aligned}
\frac{\partial E}{\partial f}= & f \cdot F\left[M F f^{2}-g\right]^{*}+f \cdot F^{*}\left[M F f^{2}-g\right] \\
& -2 \lambda f \cdot\left[\nabla_{x} S^{\prime}\left(\nabla_{x} f^{2}\right)+\nabla_{y} S^{\prime}\left(\nabla_{y} f^{2}\right)\right]
\end{aligned}
$$

\subsection{Total Variation (TV) Based Image Restoration}

TV regularization plays a very important role in image processing, which guarantees stable and visually good results, as it preserves sharp edges while resulting in very smooth images. However, solving the TV regularization problem analytically is not easy, since the regularization term is not differentiable. Here we employ the anisotropic form version of $\mathrm{TV}$, which is defined by

$$
T V(f)=\sum_{i, j}\left\|\nabla_{x} f\right\|_{1}+\left\|\nabla_{y} f\right\|_{1}
$$

where $\nabla_{x}, \nabla_{y}$ are defined by $\nabla_{x} f(i, j)=f_{i, j}-f_{i, j-1}$ and $\nabla_{y} f(i, j)=f_{i, j}-f_{i-1, j}$. Based on the above TV model, the objective function for image restoration can be written as

$$
E(f)=\frac{1}{2}\|H f-g\|_{2}^{2}+\lambda T V(f)
$$

Similarly, the smoothed goal function can be written as

$$
E(f)=\frac{1}{2}\|H f-g\|_{2}^{2}+\lambda \sum_{i, j}\left[S\left(\nabla_{x} f\right)+S\left(\nabla_{y} f\right)\right]
$$

and its analytic gradient function is

$$
\frac{\partial E}{\partial f}=(H f-g)-\lambda\left[\nabla_{x} S^{\prime}\left(\nabla_{x} f\right)+\nabla_{y} S^{\prime}\left(\nabla_{y} f\right)\right]
$$

\subsection{A Simple TV-Sparse Constraint by Thresholding}

The problem of the smoothened $l_{1}$-norm regularization is that the solution is not per- 
fectly sparse anymore, since the range near zeros becomes smooth. However, the approximation of the smoothed function is still good enough to represent sparsity in many applications such as image restoration problems.

For strict TV sparsity regularization, another sparsity constraint can be introduced. The idea is to employ a Haar wavelet [86] basis to enforce TV sparsity. The constraint can be implemented by minimizing the cost function

$$
E(f)=\|\nabla f-T(\nabla g)\|_{2}^{2}+\|W(f)\|_{0}
$$

where $T$ denotes thresholding, $g$ denotes given image solving by proposed algorithm, and $W$ denotes a wavelet transform. In practice, solving a $l_{0}$-norm regularization optimization problem is very challenging. Instead, one can solve by an iterative shrinkage thresholding algorithm (ISTA) [87]. The algorithm can be implemented as shown by algorithm 6.1.

\section{Algorithm 6.1}

Initialize $f_{j=0}$;

while: $\left\|f_{k+1}-f_{k}\right\|>\epsilon_{1}$

$f_{j}=\operatorname{argmin} E(f)$;

$f_{k=0}=f_{j}$

while: $\left\|f_{k+1}-f_{k}\right\|>\epsilon_{2}$

$f_{k+1}=\operatorname{argmin}\left\|\nabla f_{k}-T\left(\nabla f_{j}\right)\right\|_{2}^{2} ;$

$f_{k+1}=W^{-1} T\left[W\left(f_{k+1}\right)\right]$;

$k=k+1$;

\section{End}

$f_{j+1}=f_{k+1}$;

$j=j+1$

\section{End}

In detail, $f_{j}$ is solved by the proposed algorithm, and the sub-problem

$$
f=\operatorname{argmin}\|\nabla f-T(\nabla g)\|_{2}^{2}
$$

This can be solved by existing optimization methods like the steepest descend method or the conjugate gradient method. The convergence of ISTA has been shown in Ref. [88]. Simulations show that ISTA is a very efficient approach to generate sparse solutions.

\subsection{Results}

Based on the proposed models above, I verified the proposed method and checked the performance of existing solvers for some important applications. The configuration of the computer used was: 17-6700HQ CPU@2.6GHz, 16G RAM, 64-bit Windows 10 OS and Matlab R2015b. 
Firstly, I checked the proposed algorithm on a very basic problem, the basis pursuit problem. The simulation is based on "11qc_example.m" in L1-Magic. The ground-truth signal contains 5120 samples, but only 1200 samples referred to as measurement data are used for reconstruction. Gaussian random noise is added to the simulated data. Smoothed function (2), BFGS algorithm is used to solve this 1D signal CS reconstruction problem $(\mu=0, \sigma=50)$.

(a)

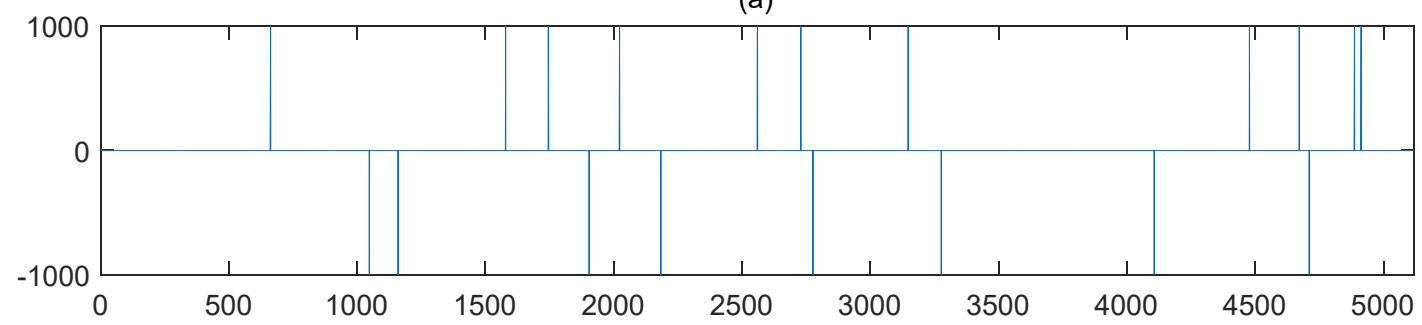

(b)
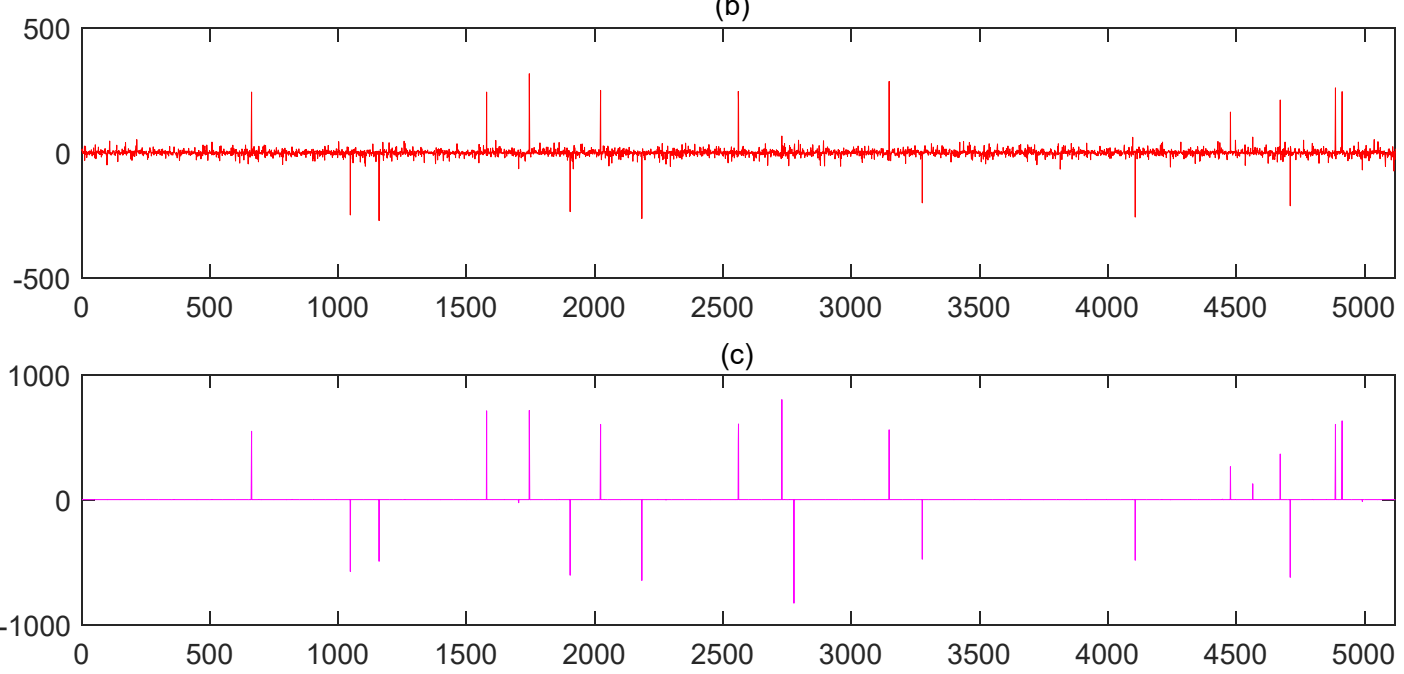

Figure 6.1 Comparison of 1D signal CS reconstructions. (a) Original signal, (b) result by L1Magic code, (c) result by the proposed model solved by LBFGS algorithm

The results show that the accuracy of both methods is quite close, but the L1-Magic solver is faster. In our simulation environment, L1-Magic takes about $9 \mathrm{~s}$, while my proposed method needs around $6 \mathrm{~s}$. However, L1-Magic results in more noisy data. In contrast, the non-linear based method is much more robust. Therefore, the non-linear based reconstruction method is very promising for CS reconstruction in practice.

I further tested the algorithm on a TV sparse CS problem, which is widely used in many applications. The test is based on the L1-Magic sample of "tveq_phantom_example.m". In L1-Magic, the problem is solved by a program coded by Justin Romberg.

The phantom image used for testing is shown in Figure 6.2. CS data is measured in Fourier domain, which is complex-valued. Only 12 radial lines in the Fourier domain are used to select a sub-set of the whole domain, which is equivalent to a random sampling. Only 2741 pixels in total are selected for reconstruction, which accounts for $4.18 \%$ of the full number of pixels. The initial guess of the object is estimated by an inverse Fourier transform of the incompletely sampled Fourier domain image. 

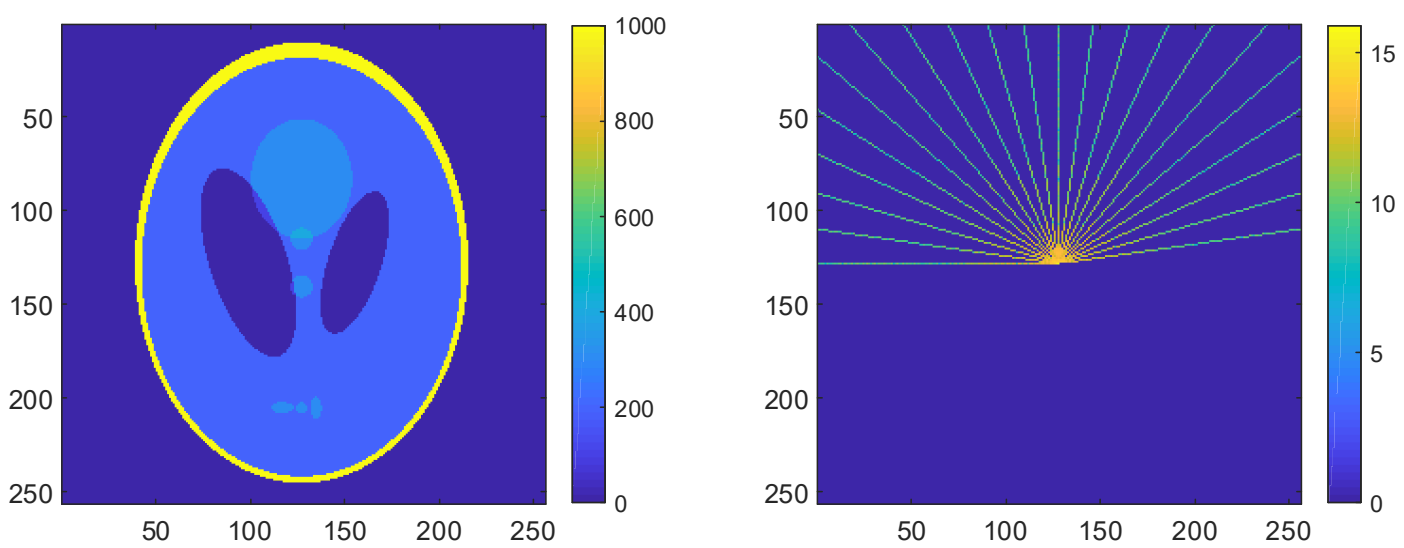

Figure 6.2 Left: ground-truth image. Right: the Fourier sampling pattern is added and the amplitude of spectrum has been logarithm-scaled (base $=10$ ).

The structure of the object is very simple. The ground-truth image consists of several phantom patterns, which are very sparse in TV domain, as can be seen in figure 6.3.
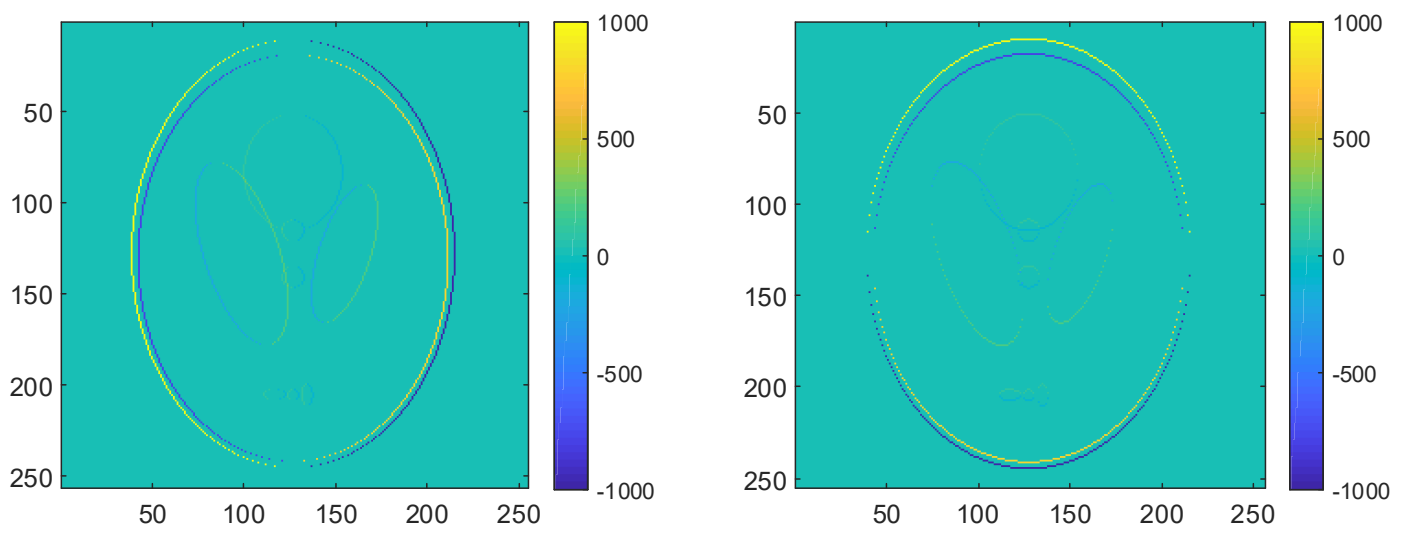

Figure 3 The TV sparsity of the object. Left: gradient in X-direction, right: gradient in Y-direction

The initial image and final result which was restored by using my proposed method is shown in Figure 4.
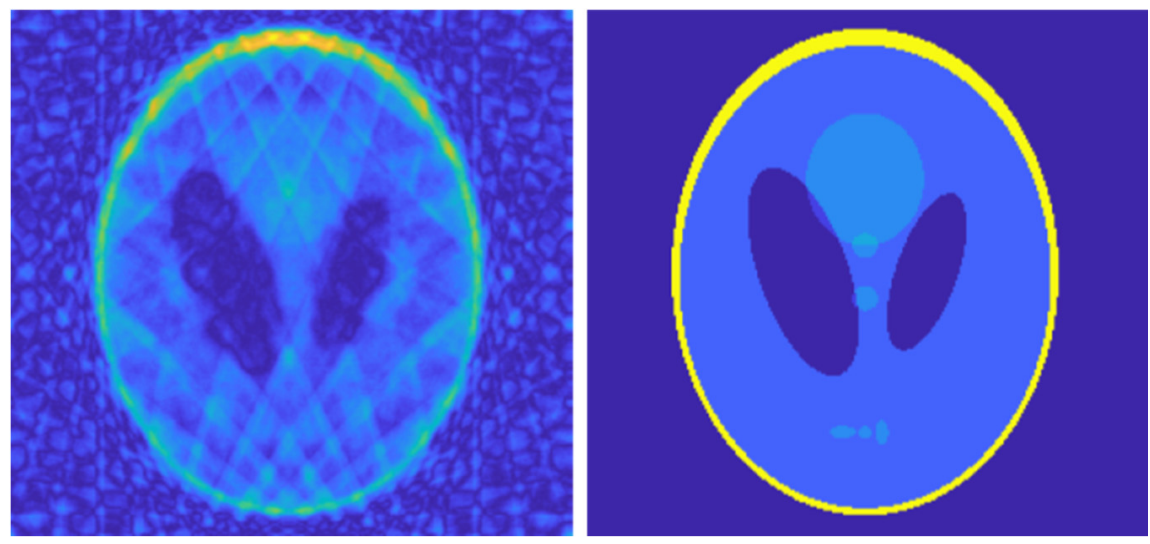

Figure 6.4 Phantom CS reconstruction. Left: initial estimated image. Right: reconstructed image by my proposed method. 
In figure 6.4, the simulation results show that both of L1-Magic and my proposed method work quite well. However, the L1-Magic solver results in negative values, while my proposed method gives always only non-negative values, which is a very useful constraint for image restoration. The following Table shows that the proposed reconstruction method significantly outperforms L1-Magic.

Table 4.1 CPU time to meet the criteria of $\mathrm{RMS}=0.1116$

\begin{tabular}{|c|c|c|c|}
\hline Algorithm & L1-Magic & LBFGS & LBFGS+ISTA \\
\hline CPU Time & $317.740 \mathrm{~s}$ & $26.369 \mathrm{~s}$ & $194.623 \mathrm{~s}$ \\
\hline
\end{tabular}

In order to study what the optimal parameter $\lambda$ is for regularization, and which effect other parameter values, e.g. the amplitude $A$ and $\sigma$, have on the the RMS of the difference between the restored image and the ground-truth image, the proposed algorithm was tested with different value combinations of these parameters. The test result is presented in Figure 6.5.

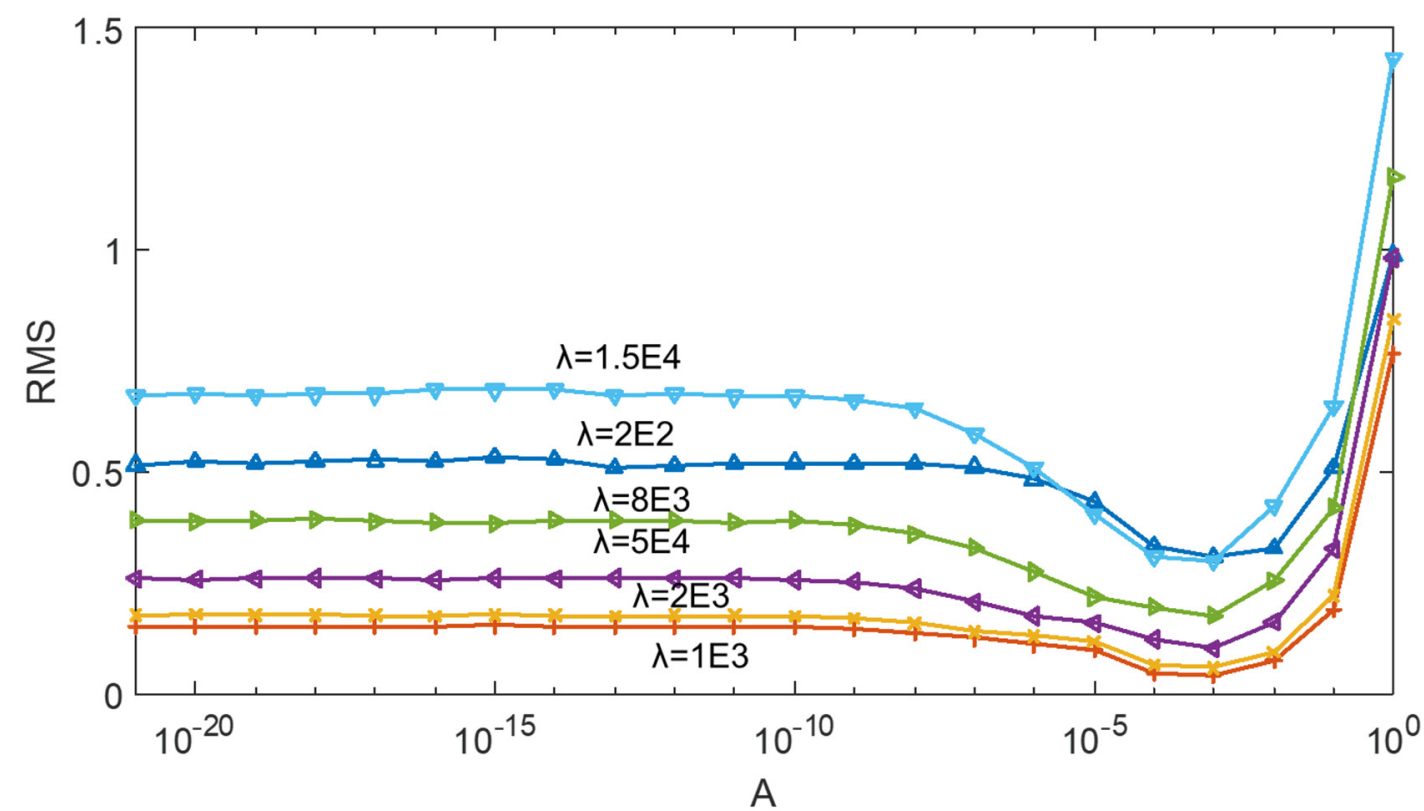

Figure 6.5 Amplitude VS RMS. The value of sigma of Gaussian function is set as $\sigma=1$.

The result shows that the RMS becomes minimal when $A=10^{-3}$; the RMS converges to a constant value when $A<10^{-10}$. The RMS rises dramatically when $A>10^{-1}$. It also shows that the RMS value becomes minimal when the regularization parameter takes the value $\lambda=10^{3}$, and it increases when $\lambda$ is smaller or larger than $10^{3}$. Therefore, the optimal value of $\lambda$ should be around $10^{3}$. However, it must be pointed out that the optimal parameter value depends on the noise and the sparsity level. In practice, noise and a priori information of the object structure is usually not available. Therefore, it is not easy to estimate the regularization parameter, and parameter tuning is usually required. Estimation of the regularization parameter needs to be studied in future work. 


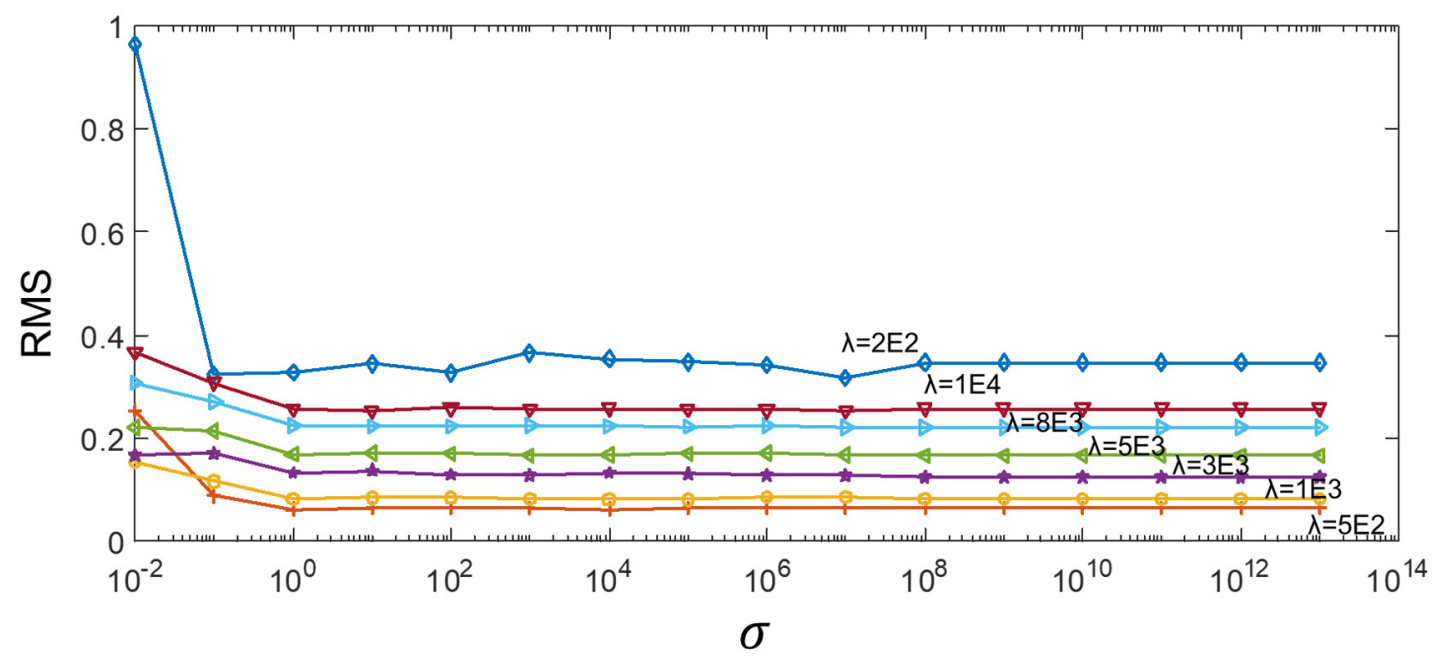

Figure 6.6 RMS vs. $\sigma$

Figure 6.6 shows the effect of $\sigma$ on RMS. It shows that the RMS value converges to a constant when $\sigma \geq 10^{8}$; the RMS value fluctuates slightly in the range of $\left[1,10^{8}\right]$, and it starts to increase with decreasing value of sigma for $\sigma<1$. Therefore, selecting a value of sigma larger than one may guarantee a good result. Both figures show that $\lambda=10^{3}$ is a good option.
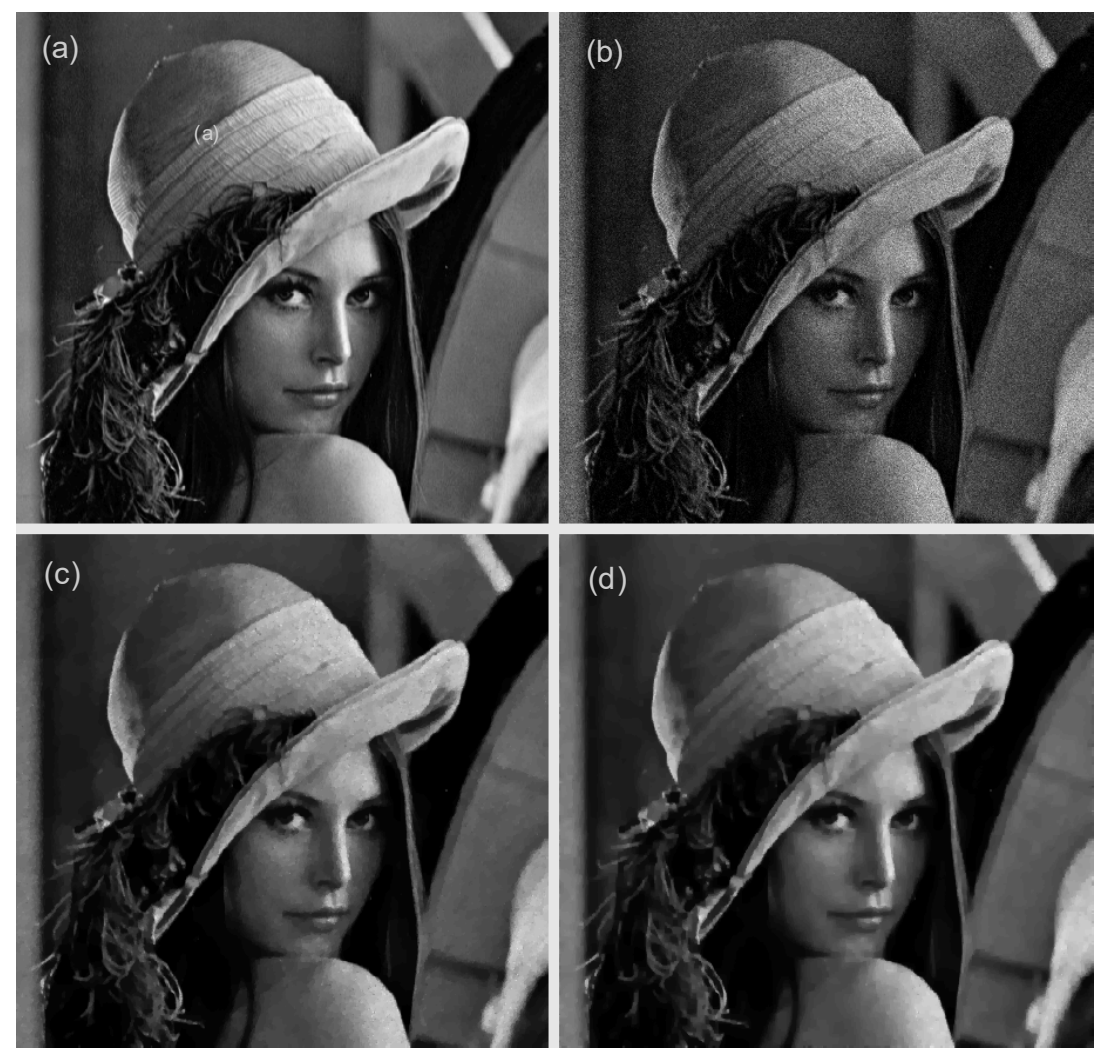

Figure 6.7 TV Image restoration. Poisson noise is added to original image (Intensity $\leq 100$ ). (a) ground-truth image, (b) noisy image, (c) denoised image by Tom's algorithm [81], (d) denoised image by proposed method solved by LBFGS algorithm. 
I also tested my proposed algorithm on image restoration based on TV regularization, which is a very widely used technique in image restoration, because it results in high contrast and very sharp edges even for very noisy images. Figure 6.7 shows that the proposed method successfully restores the ground-truth image from very noisy image and that the result is quite close to that of existing methods; both restored images have very close SNR.

\subsection{Conclusions}

In this chapter, a non-linear optimization based method for solving $l_{1}$-norm regularized optimization in CS and image restoration is proposed. It introduces a smooth function to approximate the $l_{1}$-norm, so that it can be converted to a non-linear optimization problem and then be solved by existing powerful solvers. The results show that it is a very flexible approach, any type of $l_{1}$-norm regularized optimization problem can be solved easily by existing nonlinear optimization methods via such simple conversion. I verified the proposed method by solving several existing important problems of practical relevance. The simulation results show that for basis-pursuit problems and TVsparse based CS reconstruction problems, the proposed method results in higher SNR than existing methods. Its property of accurate reconstruction makes it very suitable for CS reconstruction in practice. I also applied the method to a TV image restoration problem. The results are very close to those of existing methods. Future work has to be done for accelerating the calculations by using non-linear optimization methods to solve large-scale CS reconstruction problems. 


\section{Chapter 7}

\section{Artifact Removal Deconvolution}

\subsection{Introduction}

Regularization-based deconvolution plays a very important role in the field of imaging, because it significantly improves the quality of measured images. Even though many commercial and open source deconvolution software packages [45] for bio-imaging have been developed, many new advanced deconvolution schemes are still being developed. In Ref. [89], a Richardson-Lucy algorithm with total variation regularization was proposed for deconvolution and image smoothening in 3D confocal microscopy. It is well known that TV regularization is a very successful technique for image restoration, because it preserves sharp image edges, strongly suppresses noise, and results in visually good images. It works very well also for non-continuous images. The drawback of TV regularization is that it produces staircasing effects for images that contain smooth but complex structures. This limitation of TV has inspired regularizations based on higher order derivatives: Hessian regularization.

Hessian regularization is a very good alternative to TV regularization, due to its global smoothening property for continuous images. Ref. [90] proposes an algorithm that is based on a Majorize-Minimization method to solve Hessian-regularization-based deconvolution problems, and its results demonstrated its potential for applications in bioimaging. The drawback is that the algorithm does not contain a non-negative constraint. Recently, a fast, long-term, super-resolution imaging method was reported: HessianSIM [13]. Here, a Hessian regularization artifact-removal deconvolution has been proposed that is based on the Split-Bregman method. It takes advantage of the smoothening property of Hessian regularization without harming super-resolution. It makes sense to employ Hessian regularization because the object image in bio-imaging is supposed to be continuous and smooth. From the results of Hessian-SIM, it can be seen that Hessian-based regularization is quite promising for bio-imaging.

In this chapter, I will explain the basic concept of deconvolution and introduce some important existing techniques for fluorescence microscopy. Then I derive the deconvolution formula for Maximum a Posteriori (MAP) based deconvolution with Hessian regularization and roughness regularization [91]. Based on simulation and experiment with real sample image, I show that Hessian regularization can also be used for intrinsic artifact removal in super-resolution microscopy as developed in our group, e.g. SOFI and ISM. I also propose a flexible method to solve Hessian-regularized deconvolution problems, which takes a non-negative constraint into consideration and is based on existing solvers, by which, it is much easier to implement. 


\subsection{Concept of Deconvolution and some Basic Decon- volution Methods}

Mathematically, deconvolution is based on the fact that, for a linear imaging system, the process of imaging is equivalent to convolving the sample image $f$ with a point spread function (PSF) $H$ :

$$
G=H * f+N
$$

It is convenient to use a blurring matrix $H$ to represent the PSF for analysis. Then, (7.1) can be rewritten by $G=H f$. Given the PSF $H$ and the measured degraded image $G$, deconvolution can be ideally done by

$$
f=H^{-1} \cdot G
$$

which is indeed a very simple linear operation. The problem is that the blurring matrix is ill-defined. Therefore, such an inverse filter cannot be used in practice when noise is involved. When the blurred image is noisy, the inverse of the PSF will result in its amplification ate high frequencies. A simple way to avoid this problem is to introduce a damping factor to control the amplification amplitude of noise at high frequencies. The optimal solution based on a Minimum Mean-Square Error (MMSE) results in

$$
f(\boldsymbol{k})=\frac{H^{*}(\boldsymbol{k}) G(\boldsymbol{k})}{H^{*}(\boldsymbol{k}) H(\boldsymbol{k})+N / S}=\frac{H^{*}(\boldsymbol{k}) G(\boldsymbol{k})}{H(\boldsymbol{k}) H^{*}(\boldsymbol{k})+1 / S N R}
$$

which is also called a Wiener filter, where $N$ denotes spectrum of the noise, $S$ is the signal spectrum, and SNR denotes the signal-to-noise ratio. When the SNR is high, (7.3) turns into (7.2). When the SNR is small, the filter value tends to zero. In other words, with high SNR, the Wiener filter works as an inverse filter, and with low SNR, the Wiener filter suppresses noise amplification. The problem of the Wiener filter is that it requires knowledge of the exact spectra of noise and signal, which are usually not available in practice. Instead, a constant can be used to approximate the SNR.

By introducing a regularization parameter $\lambda$, the we can obtain the following form

$$
f=\frac{H^{T} G}{H^{T} H+\lambda I}
$$

which is called a Tikhonov regularized inverse filter. The regularization parameter $\lambda$ is the value of the trade-off between high frequency noise amplification and noise suppression. The optimum choice of $\lambda$ is key for obtaining a good deconvolution result. In fact, (7.4) can also be solved iteratively with some efficient algorithm such as a conjugate gradient method. In practice, a non-negative constraint is introduced in each iteration, which is then called an Iterative Constrained Tikhonov-Miller (ICTM) method [92]. The details of ICTM have been discussed in the second chapter.

Another well-known deconvolution method is the Richardson-Lucy algorithm (RL), which is a non-linear deconvolution technique that assumed a Poisson distribution of the image noise. Its very simple iteration is given by 


$$
f_{n+1}=f_{n} \cdot\left(H^{T} * \frac{G}{H * f}\right)
$$

This iteration of the RL algorithm tries to search a solution that maximizes the expectation of a Bayesian estimate. The Richardson-Lucy algorithm is very easy to implement, it does not require any regularization parameter, and it is much more robust than the linear deconvolution techniques mentioned above. Furthermore, a non-negative constraint is already implicitly introduced in the RL algorithm.

The advantages of these classical deconvolution methods are their high efficiency and easy implementation. A drawback is that artifacts arise for very noisy images. To suppress noise and artifacts, a regularization is required.

\subsection{Maximum a Posteriori Estimation Based Deconvo- lution with Regularization}

The Maximum a Posteriori technique is an advanced technique for florescence microscopy deconvolution. Theoretically, MAP finds a solution that matches the observed data with highest statistical probability. Verveer has given the details of MAP for deconvolution problem in his thesis [48]. Here, I just show some important conclusions. I consider MAP with two type of noise models, a Gaussian noise model MAP (MAPG) and Poissonian noise model MAP (MAPP).

For Gaussian noise, MAP results in a cost function of

$$
E(f)=\frac{1}{2}\|H f-G\|_{2}^{2}+\lambda R(f)
$$

where $f$ denotes the object function, $R(f)$ denotes regularization, and $\lambda$ denotes Lagrange multiplier. For an iterative scheme, an analytic gradient function is essential for calculation. The gradient of MAPG can be obtained by

$$
\frac{\partial E}{\partial f}=H^{T}(H f-g)+\lambda \frac{\partial R}{\partial f}
$$

where $T$ denotes transpose of matrix.

Total Variation regularization is a very commonly used classical regularization technique for image denoising. The definition of Total Variation regularization is

$$
R(f)=\|\nabla f\|_{1}
$$

where $\nabla$ denotes the gradient operator, which corresponds to a high-pass filter such as taking difference in the $x$ - and $y$-direction. In the case of $R(f)=\left\|\nabla_{\mathrm{x}} f\right\|_{1}+\left\|\nabla_{\mathrm{y}} f\right\|_{1}$, the Gaussian MAP results in

$$
E(f)=\frac{1}{2}\|H f-G\|_{2}^{2}+\lambda\left(\left\|\nabla_{\mathrm{x}} f\right\|_{1}+\left\|\nabla_{\mathrm{y}} f\right\|_{1}\right)
$$

Here, the multiplier $\lambda$ actually controls the tradeoff threshold, which balances the high resolution and smoothness of the deconvolved image. 
For a Poissonian noise statistics, MAP results in the cost function

$$
E(f)=\sum_{i}[H f-G \cdot \ln (H f+b)]_{i}+\lambda R(f)
$$

where $i$ denotes the pixel index, and $b$ is the background of the measured image. Without any regularization term, MAPP will yield the same solution as the RL algorithm. The gradient of MAPP is found to be

$$
\frac{\partial E}{\partial f}=1-H^{T}\left(\frac{G}{H f+b}\right)+\lambda \frac{\partial R}{\partial f}
$$

The selection of the regularization parameter is essential for the performance of regularized deconvolution. Basically, the regularization value can be determined as

$$
\lambda=\frac{\sigma_{N}^{2}}{\sigma_{L}^{2}}
$$

where $\sigma_{N}^{2}$ is the noise variance of the measured image, and $\sigma_{L}^{2}$ is the variance of the regularized image. In practice, $\sigma_{N}^{2}$ can be measured by experiment or estimated from a measured image. But $\sigma_{L}^{2}$ is usually difficult to determine, because the structure of the image is not known, so parameter tuning is usually needed. Typical values for $\lambda$ are in the interval $\left[10^{-4}, 10^{-2}\right]$.

There are many regularization techniques are available. Popular regularizations for image restoration are Total Variation (TV), Tikhonov regularization, Wavelet sparsity etc. The choice of regularization has a great impact on the performance of a deconvolution algorithm. The regularization selection has to consider the properties of the selected technique and the structure of the object. For example, Tikhonov regularization is more suitable to smooth images, whereas TV regularization is more suitable for staircasestructured images.

In this chapter, I study the Hessian regularization and Roughness regularization, which have been demonstrated to be very attractive for bio-imaging deconvolution.

\subsection{Hessian Regularized Deconvolution}

The Hessian regularization function for image restoration can be defined by

$$
\operatorname{Hess}(f)=\sum_{i j}\left|\nabla_{x x} f\right|+\left|\nabla_{y y} f\right|+2\left|\nabla_{x y} f\right|
$$

where $\nabla_{x x}, \nabla_{y y}, \nabla_{x y}$ are given by

$$
\left\{\begin{array}{l}
\nabla_{x x} f(i, j)=f_{i-1, j}-2 f_{i j}+f_{i+1, j} \\
\nabla_{y y} f(i, j)=f_{i, j-1}-2 f_{i j}+f_{i, j+1} \\
\nabla_{x y} f(i, j)=f(i, j)-f(i, j-1)-f(i-1, j)+f(i-1, j-1)
\end{array}\right.
$$

Instead, only $\nabla_{x x}, \nabla_{y y}$ can be used, which yields a $l_{1}$-norm Laplace regularization. Based on the above Hessian regularization model, the goal function of image deconvolution can be represented as

$$
E(f)=\frac{1}{2}\|H f-g\|_{2}^{2}+\lambda H e s s(f)
$$


The function of $l_{1}$-norm is not differentiable, however, it is possible to approximate it by some smooth function that can guarantee that the object function will be smooth everywhere while the output of each element term in the $l_{1}$-norm function is positivevalued.

$$
R(s)=\|x\|_{1} \cong \sum_{i} S\left(x_{i}\right)
$$

The $l_{1}$-norm function can be approximated by $\|x\|_{1}=\sqrt{f(x)+x^{2}}$, where $f(x)$ denotes a positive decay function from the origin to $\pm \infty$. Indeed, there are many functions having this positivity and decay property, for example, the reciprocal square function

$$
S(x)=\sqrt{x^{2}+\frac{A}{x^{2}+1}}
$$

where $A$ denotes the amplitude of a regularization term. Its derivative is

$$
S^{\prime}(X)=\frac{x\left[1-\frac{A}{\left(x^{2}+1\right)^{2}}\right]}{\sqrt{x^{2}+\frac{A}{x^{2}+1}}}
$$

The smoothened goal function can be written as

$$
E(f)=\frac{1}{2}\|H f-g\|_{2}^{2}+\lambda \sum_{i, j} S\left(\nabla_{x x} f\right)+S\left(\nabla_{y y} f\right)+2 S\left(\nabla_{x y} f\right)
$$

and its gradient is

$$
\frac{\partial E}{\partial f}=H^{T}(H f-g)+\lambda\left[\nabla_{x x} S^{\prime}\left(\nabla_{x x} f\right)+\nabla_{y y} S^{\prime}\left(\nabla_{y y} f\right)+2 \nabla_{x y}^{*} S^{\prime}\left(\nabla_{x y} f\right)\right]
$$

where $\nabla_{x y}^{*}$ is defined by

$$
\nabla_{x y}^{*} f(i, j)=f(i, j)-f(i, j+1)-f(i+1, j)+f(i+1, j+1)
$$

which is very similar to $\nabla_{x y}$.

In image deconvolution, a non-negative constraint is a very useful condition for strengthening a deconvolution algorithm. It is very easy to impose non-negative constrain by substituting $f$ with $f^{2}$ in the above equations. Then, the non-negative constrained deconvolution goal function becomes

$$
E(f)=\frac{1}{2}\left\|H f^{2}-g\right\|_{2}^{2}+\lambda H \operatorname{Hess}\left(f^{2}\right)
$$

which is equivalent to

$$
E(f)=\frac{1}{2}\left\|H f^{2}-g\right\|_{2}^{2}+\lambda \sum_{i, j} S\left(\nabla_{x x} f^{2}\right)+S\left(\nabla_{y y} f^{2}\right)+2 S\left(\nabla_{x y} f^{2}\right)
$$

and the corresponding gradient can be easily derived by

$$
\begin{aligned}
\frac{\partial E}{\partial f}= & 2 f^{T} H^{T}(H f-g) \\
& +2 \lambda f^{T}\left[\nabla_{x x} S^{\prime}\left(\nabla_{x x} f^{2}\right)+\nabla_{y y} S^{\prime}\left(\nabla_{y y} f^{2}\right)+2 \nabla_{x y}^{*} S^{\prime}\left(\nabla_{x y} f^{2}\right)\right]
\end{aligned}
$$

Now, the Hessian-regularization based deconvolution problem has been converted to a 
non-linear unconstrained optimization problem, which can be solved by many existing large-scale non-linear optimization methods, such as LBFGS, non-linear conjugate gradient etc.

\subsection{Roughness Regularization}

Roughness regularization is defined by

$$
R_{R F}(f)=\int \frac{|\nabla f(X)|^{2}}{f(X)} d X
$$

where $X$ denotes any direction. A forward difference scheme can be used to approximate the gradient, i.e.

$$
R_{R F}^{X}(f)=\sum_{n} \frac{\left(f_{n+1}-f_{n}\right)^{2}}{f_{n}}
$$

The corresponding gradient reads

$$
\nabla_{X} R_{R F}=\frac{\partial R_{R F}^{X}}{\partial f_{n}}=-\frac{2\left(f_{n+1}-f_{n}\right)}{f_{n}}-\frac{\left(f_{n+1}-f_{n}\right)^{2}}{f_{n}^{2}}+\frac{2\left(f_{n}-f_{n-1}\right)}{f_{n-1}}
$$

In case of a two-dimensional regularization, the roughness regularization function can be represented as

$$
R_{R F}(f)=R_{R F}^{x}(f)+R_{R F}^{y}(f)
$$

The corresponding gradient is

$$
\nabla R_{R F}=\nabla_{x} R_{R F}+\nabla_{y} R_{R F}
$$

Like Hessian-regularized deconvolution, the positive constraint goal function for roughness regularized deconvolution can be written as

$$
E(f)=\frac{1}{2}\left\|H f^{2}-g\right\|_{2}^{2}+\lambda R F\left(f^{2}\right)
$$

The gradient can be derived as

$$
\frac{\partial E}{\partial f}=2 f^{T} H^{T}\left(H f^{2}-g\right)+2 \lambda f^{T} \cdot \nabla R_{R F}\left(f^{2}\right)
$$

\subsection{Simulation}

Simulations were implemented to verify the performance of the regularized deconvolution algorithms studied for 2D and 3D image deconvolution. Since the image noise is dominated by Poissonian noise, I have chosen the MAPP model and implemented a deconvolution algorithm based on (7.10) and (7.11). The problem of minimizing (7.10) was solved with a LBFGS algorithm. 


\subsubsection{Two-Dimensional image deconvolution}

In order to check the performance of the proposed methods, I firstly tested the algorithms on the resolution target from website [93]. The configuration of the computer used for these simulations was: i5-6600 CPU@3.30GHz, 32G RAM, 64-bit Windows10 OS, and the platform for coding and testing is based on Matlab R2017a.

The image was blurred with a Gaussian kernel, and Poisson noise was added. The goal function is based on (7.10), which is solved by LBFGS algorithm.

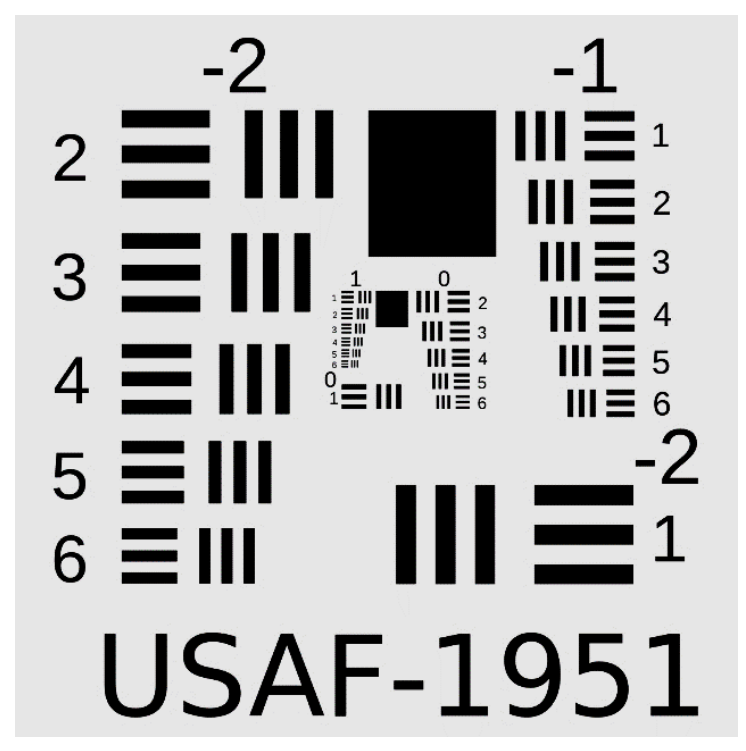

Figure 7.1 Ground-truth

In order to quantify the performance of each algorithm, I employed the root-meansquare (RMS) deviation defined by

$$
R M S=\sqrt{\frac{\sum_{i}\left(I_{\text {deconv }}-I_{\text {truth }}\right)_{i}}{M \times N}}
$$

where $I_{\text {deconv }}$ denotes the deconvolved image, and $I_{\text {truth }}$ denotes the ground-truth image. $M$ and $N$ are the height and width of the image, respectively. No doubt, the RMS value reflects the performance of a deconvolution algorithm. In order to obtain the best results, the regularization parameter is determined by selecting the one that results in a minimum RMS value. To do so, several points of the RMS function

$$
\operatorname{RMS}\left(\lambda_{i}\right), \quad i=[1,2,3, \ldots]
$$

are calculated, then a continuous RMS function is estimated by cubic interpolation, and the minimum of the RMS function is selected as the optimal regularization parameter. For the RL algorithm, I have taken the result that yields a minimum RMS value.

The simulation result is shown in Figure 7.2. The results show that the RL algorithm yields sharper images, but numerous artifacts are generated. In contrast, regularized deconvolution with Hessian and roughness regularization result in very clear images 
with less artifacts. A quantitative comparison between different deconvolution methods is shown, based on the minimum of RMS and the edges response.
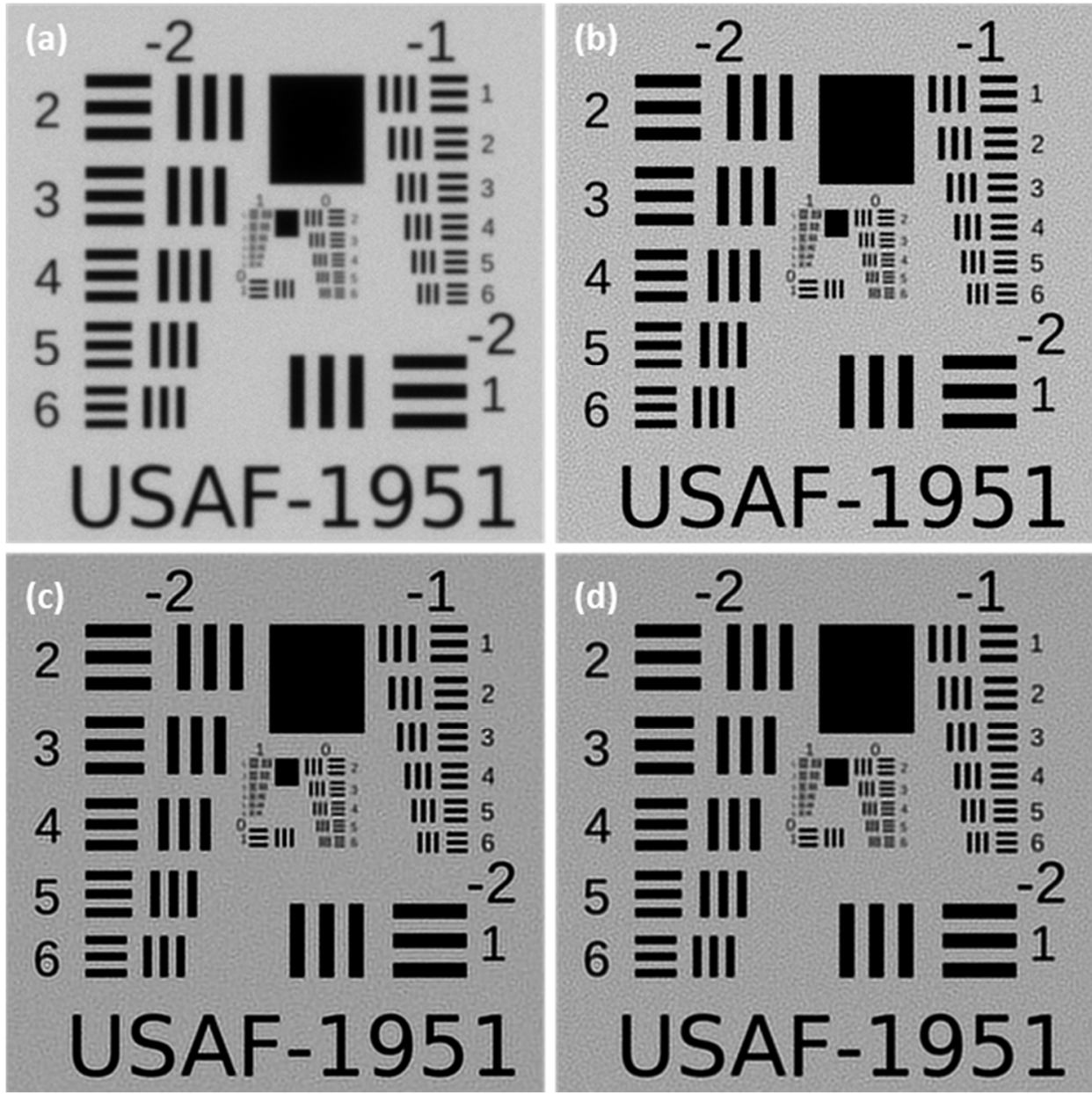

Figure 7.2 2D deconvolution. (a) Blurred noisy image, (b) RL deconvolved image, de-convolved image with (c) Hessian regularization, (d) Roughness regularization.

Table 7.1 shows RMS values for the best values of regularization parameters and number of iterations, tuned in such way as to guarantee that the deconvolution algorithms converge to the solution with minimum RMS value.

Table 7.1

\begin{tabular}{|c|c|c|c|}
\hline Regularizer & Hessian & Roughness & RL \\
\hline Parameter & 2E-3 & 2E-2 & 0 \\
\hline RMS & 25.0594 & 26.6413 & 30.2826 \\
\hline
\end{tabular}

Fig 7.3 shows details of the deconvolved images above, and a profile along the redline.
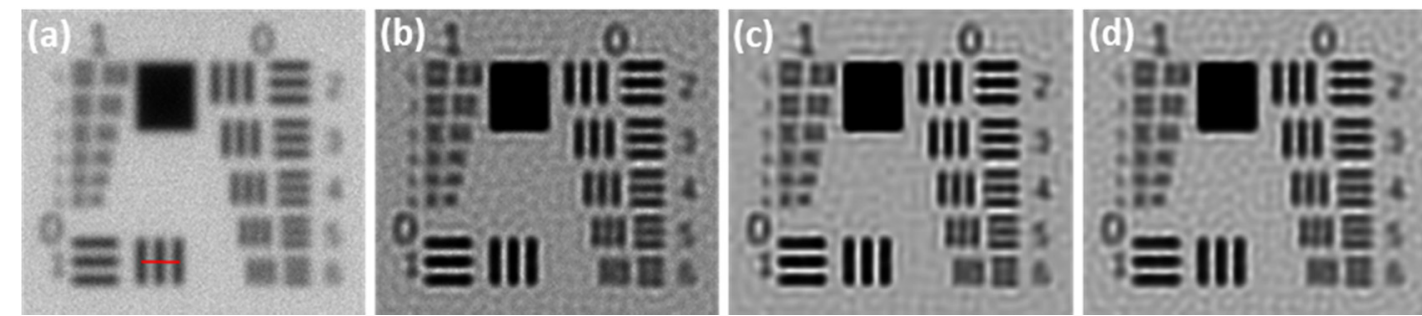


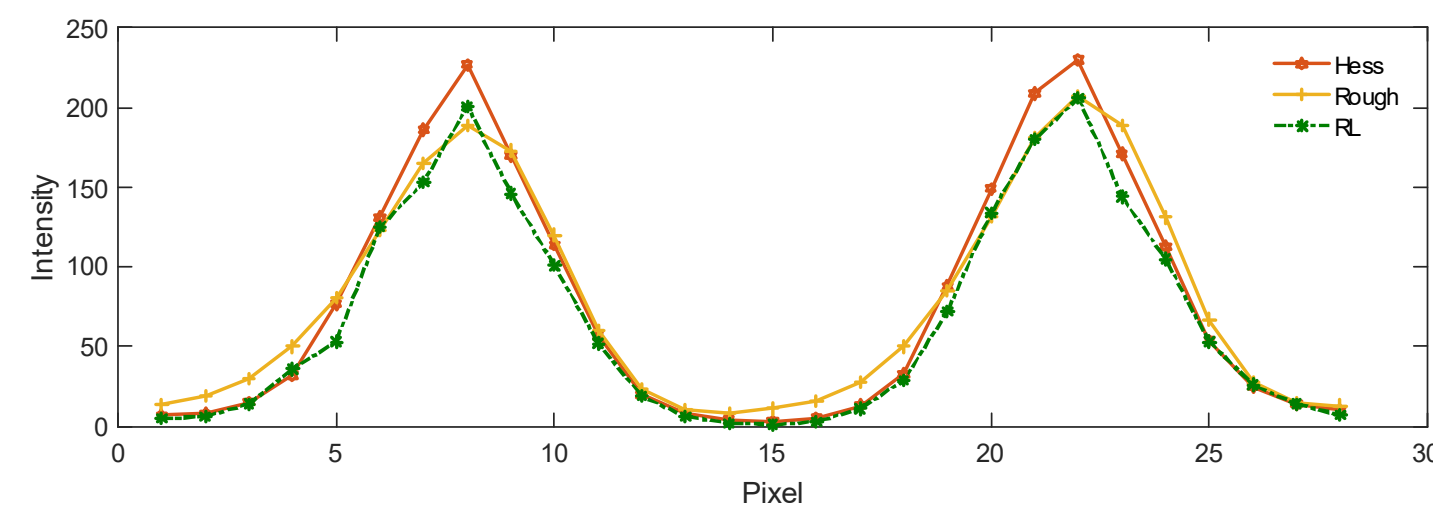

Figure 7.3 Resolution performance comparison

The curves show that the three tested methods have a very close resolution performance. However, the RL deconvolution results in the best contrast, but suffers from larger artifacts and noise. Both Hessian and Roughness regularization result in smoother and clearer images. However, there are obvious differences between these two regularizations. Hessian regularization shows a better performance in artifact removal than roughness regularization, while the intensity response at high levels of Roughness regularization is much more linear, very close to that of the RL algorithm.

\subsubsection{Three-Dimensional image deconvolution}

Three Dimensional deconvolution is a very common problem in bio-imaging. I tested 3D deconvolution with Hessian regularization and roughness regularization based on the MAPP model, using the dataset from Ref. [45]. The ground-truth image is just the one shown in figure 7.4.

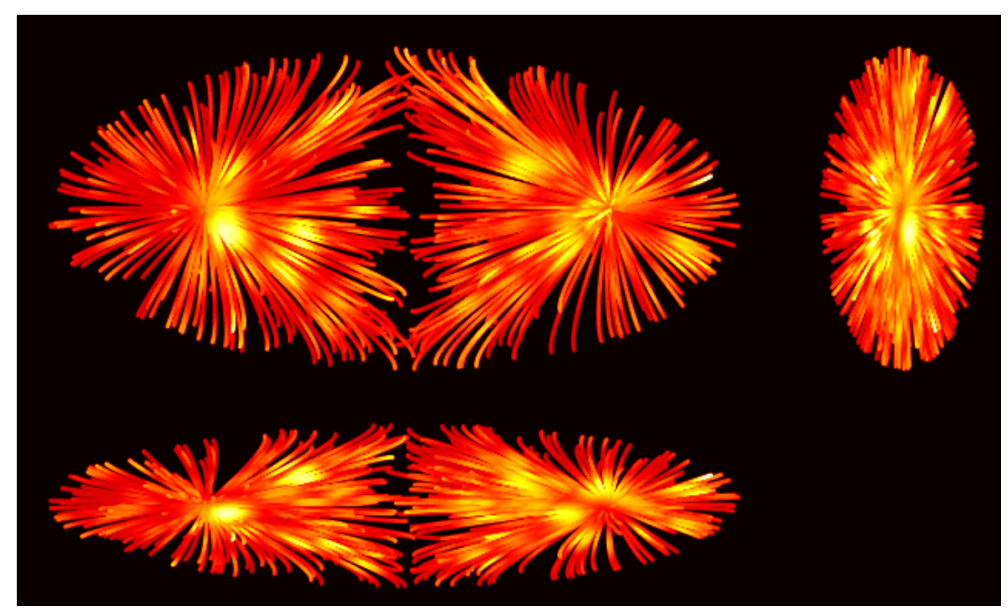

Figure 7.4 Ground-truth

The 3D image blurred by a wide-field 3D PSF and with Poisson noise added. Similarly, the cost function is based on (7.10), and LBFGS algorithm is employed to solve the problem. The simulated blurred noise image and de-convolved results are just as figure 7.5 shows. 

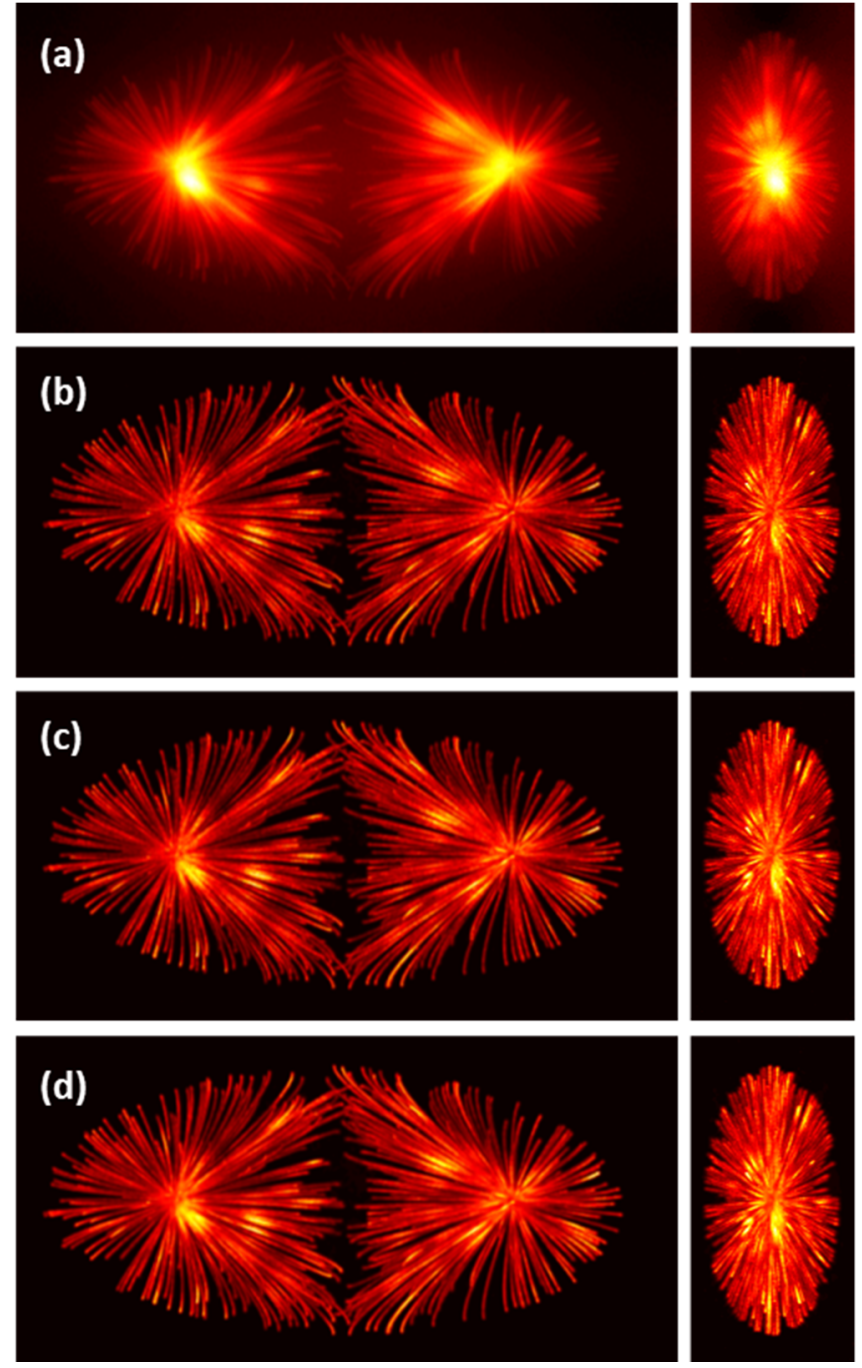

Figure 7.5 3D deconvolution. (a) Blurred noisy image, (b) RL (c) Deconvolution with roughness regularization (d) Deconvolution with Hessian regularization

For optimal regularization parameters, the resulting RMS are given in Table 7.2.

Table 7.2

\begin{tabular}{|c|c|c|c|}
\hline Algorithm/Regularizer & RL & Roughness & Hessian \\
\hline RMS & 691.1674 & 634.0935 & 640.5373 \\
\hline
\end{tabular}

Table 7.2 shows that deconvolution with regularization has a better performance than the RL algorithm. Roughness regularization seems to be a little better than Hessian regularization. The reason is that smooth structures dominate the object.

\subsection{Artifact Removal Deconvolution with Hessian Regularization}

In this section, Hessian regularization is used for artifact removal deconvolution in SDC-ISM and SOFI images, which can suffer from some intrinsic artifacts. 


\subsubsection{SOFI Image Deconvolution}

The average of raw images shows a HeLa cell with vimentin labeled by rsEGFP. I used 1000 frames of raw images to calculate the $2^{\text {nd }}$-SOFI image. The existing software developed by Theo Lasser's group at the EPFL calculates the SOFI image using crosscummulants, and then linearizes the SOFI image by taking the square-root of the deconvolved image with a RL deconvolution algorithm. I do here the same thing but use a Hessian-regularized deconvolution method to de-convolve the SOFI image. The PSF used for deconvolution is approximated by a Gaussian function whose size is based on the measured image of beads, where the measured FWHM is around 2.6 pixels.
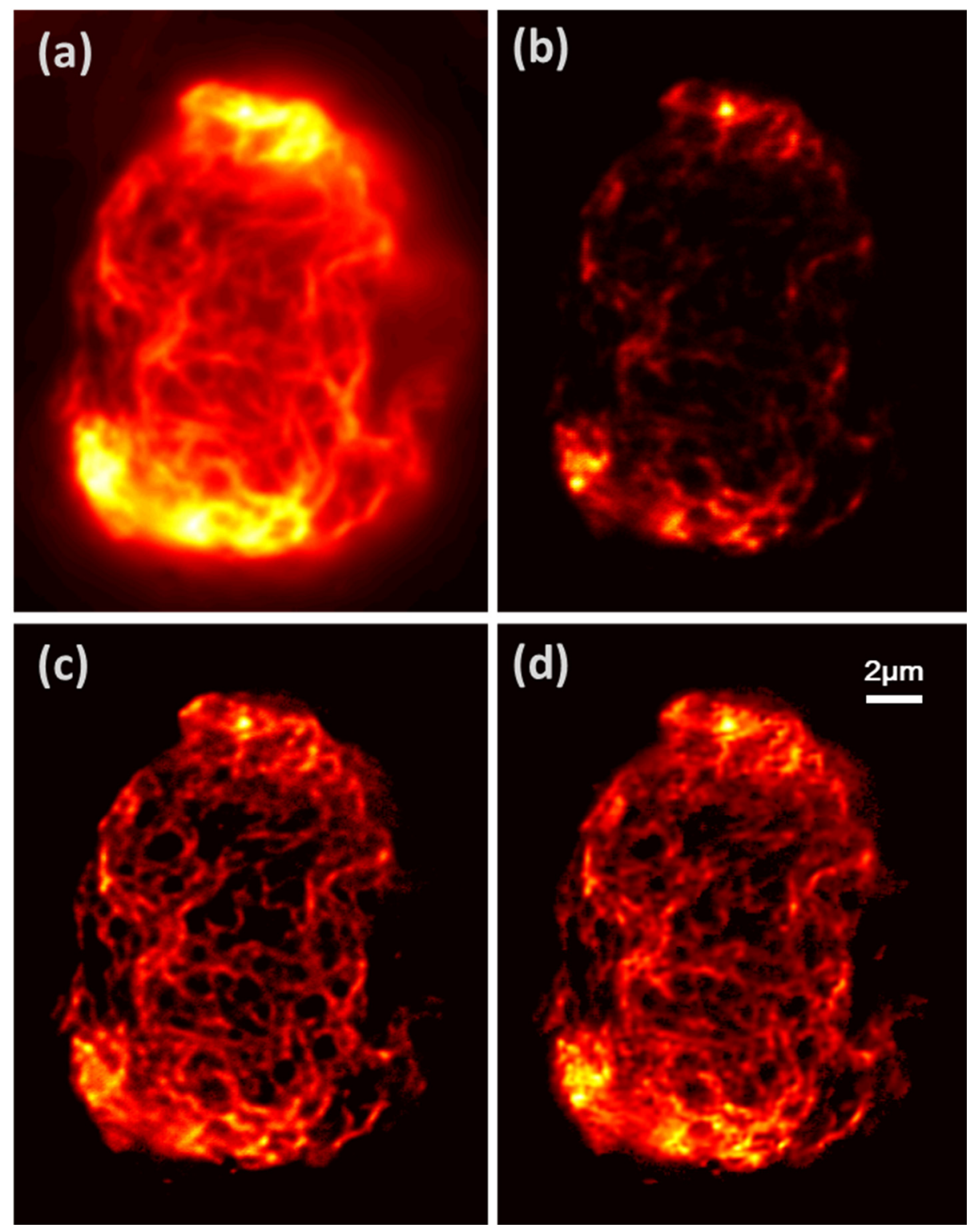

Figure 7.6 SOFI image deconvolution. (a) average image, (b) $2^{\text {nd }}$-order cross-cumulant, (c) reconstructed image by existing public-domain software [94], (d) deconvolved image by Hessian regularized deconvolution 
In order to gain a deep-insight comparison, figure 7.7 shows the details of the structure in the central area of each image in Figure 7.6.
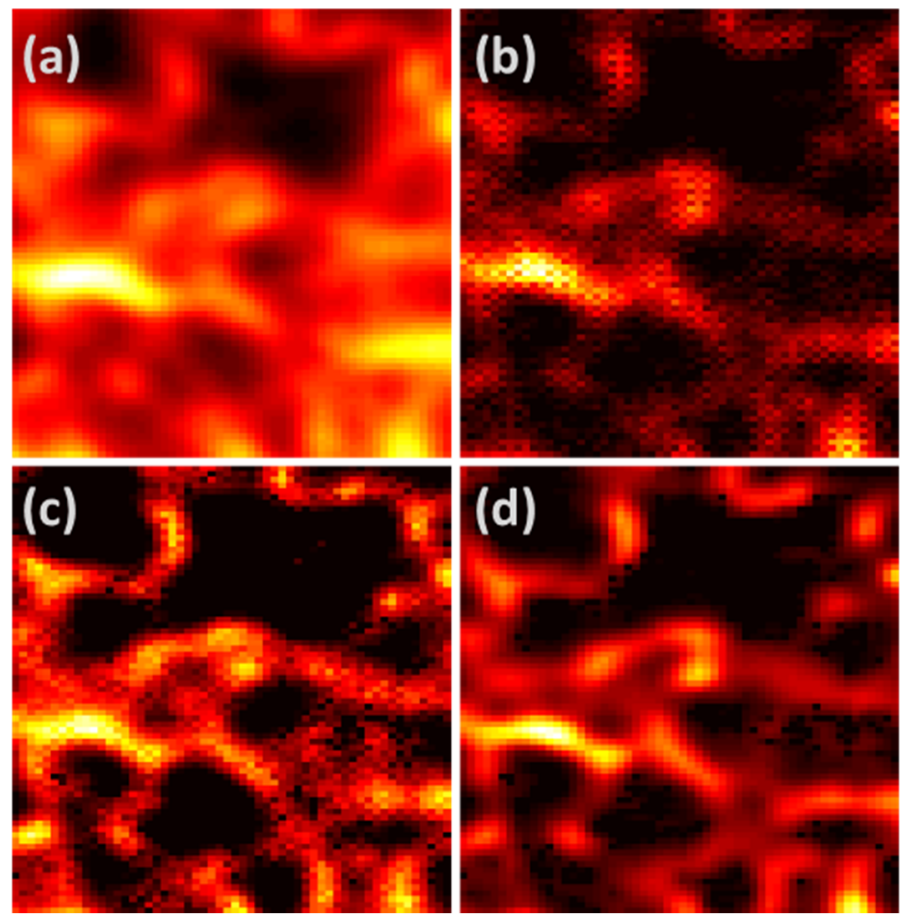

Figure 7.7 SOFI image deconvolution. (a) average image, (b) $2^{\text {nd }}$ order cross-cumulant, (c) deconvolved image by existing open software [94], (d) deconvolved image by using Hessian regularized deconvolution.

The structure and the resolution of the restored object obtained by two deconvolution methods look quite similar. However, artifacts caused by the cross-cumulant calculation cannot be removed completely by RL deconvolution, while Hessian-regularized deconvolution results in a smoother and clearer image. The reason is that, even though RL deconvolution results in a higher contrast and resolution, it is sensitive to noise. Thus, high-frequency components are also be amplified. In contrast, deconvolution with Hessian regularization does not only suppress noise very well, but also highlights connected structures in the object, and its contrast is closer to that of the average image. Therefore, Hessian regularization is very suitable for restoring image that are supposed to be continuous and smooth.

\subsubsection{SDC-ISM image deconvolution}

The image used here was obtained with the SDC-ISM system developed in our group. The sample is an EPC2 cell which is labeled with phalloidin and illuminated with a $647 \mathrm{~nm}$ laser. The image was recorded with a Hamamatsu camera (pixel size/magnification $=54.2 \mathrm{~nm}$ ). The measured FWHM of one excitation focus is 7.1 pixels. Six laser pulses are triggered for each imaged frame, and 125 frames were taken in total. The average image has the same resolution as that of conventional SDC as shown in figure 7.8 (a), and the ISM image is shown in panel (b). It is apparent that stripe artifacts appear in the reconstructed ISM image. The main reason of these artifacts is the uneven- 
spatial-sampling due to the spatial distribution of pinholes in the spinning disk. Therefore, completely correcting this type of artifact by system calibration is very difficult as information of system errors relative to these artifacts is unavailable. Even filtering by the mean of the images is quite challenging, as the frequency of artifacts overlaps with the bandwidth of the object image.
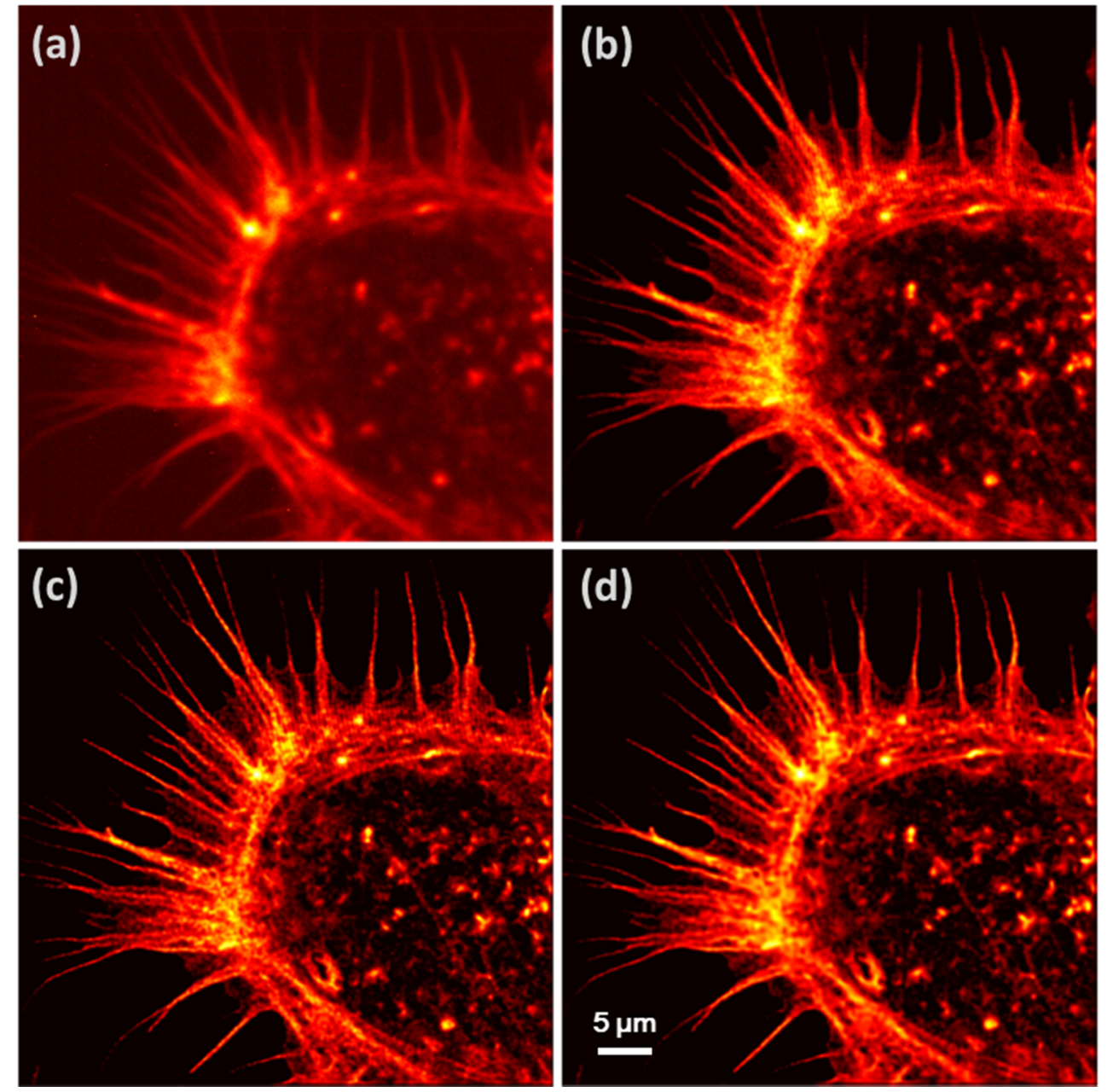

Figure 7.8 SDC-ISM image deconvolution. (a) average of raw images, (b) reconstructed ISM image, (c) deconvolved image by RL algorithm, (d) deconvolved image by Hessian-regularized deconvolution method.

The results show that artifacts are enhanced by general deconvolution methods, while Hessian-regularized deconvolution can remove the artifacts very well. However, the degree of smoothening is too big, so that high frequency information is filtered out. These two examples show that the choice of the regularization parameter is very important in practical applications. It is always a trade-off between smoothening and resolution enhancement. Unfortunately, there is no formula to determine the value of the regularization parameter. Simulations and experiment results show that a good value is around $10^{-3}$, and tuning around this value is a good option in practice. 


\subsection{Discussion}

In this chapter, I have proposed a method for enhancing resolution and removing potential artifacts in SOFI and ISM images based on a Hessian-regularized deconvolution. I showed that Hessian-regularized deconvolution can be efficiently done via non-linear optimization. The simulation results show that Hessian regularization with a proper regularization parameter results in very smooth and clear images. The results of deconvolved ISM images show that artifacts can be removed very well, but resolution is lost due to over-smoothening. Deconvolved SOFI images show that, although RL deconvolution results in high contrast images, it also enhances artifacts at the same time. Hessian-regularized deconvolution highlights the connection of the imaged object, as it assumes that the unknown object is continuous and smooth, and that is why it can restore missing structures of a sample from partial knowledge. Since the structure of a biological sample is usually continuous and smooth, this property makes it very suitable for continuous object imaging. In future work, it would be interesting to develop a GPU accelerated Hessian-regularized deconvolution based on a MLE, which is more suitable for low SNR fast 3D imaging. 


\section{Chapter 8}

\section{Airy Beam}

\section{Light-Sheet Microscopy}

\subsection{Introduction}

Light sheet fluorescence microscopy is a fast imaging technique with high performance of optical sectioning [95]. By using a laser light-sheet for excitation, background and bleaching can be reduced. Light sheet microscopy results in large Field-of-View (FOV) high contrast images $[96,97]$. In practice, Gaussian beam, Bessel beam, and Airy beam excitations have been employed for light-sheet microscopy. In comparison, Airy beam excitation outperforms Gaussian beam and Bessel beam excitation because it provides a much larger FOV. Airy beams have the following interesting properties: they are nondiffractive over very long propagation paths and they are "self-healing" during propagation [98]. Since the first observation of optical Airy beams [99], they have been extensively investigated $[100,101]$, and show promising potential for application in photonics and bio-imaging [102, 103].

Since 2014, Kishan Dolakia's group in St. Andrews, Scotland, has developed Airy beam light-sheet microscopy (ALSM) to obtain high contrast and very large FOV images [104]. As it profits from high spatial frequency content of Airy beams, the singephoton modulation-transfer-function (MTF) of ALSM is much better than that of Bessel beam light-sheet microscopy, while it retains a very good axial resolution very close to that of Gaussian light-sheet microscopy. The FOV of an Airy light sheet microscope can reach $173 \mu \mathrm{m}$, which is about 10 times larger than that of Gaussian light sheet microscopy. The latest version of ALSM provides even higher contrast images [105].

The high performance of ALSM comes with the problem of image reconstruction by deconvolution. The reason is that the sample is not only convolved by the point-spreadfunction of the microscope, but also with the Airy-beam illumination that is non-symmetric along the sectioning direction. This results in comet or flame artifacts in the measured image. These artifacts are not uniform along the optical axis, which is very different from traditional linear imaging methods. Such imaging behavior of Airy lightsheet microscopy requires that the measured image has to be deconvolved with the illumination function to restore the real image. Basically, the ALSM image can be reconstructed by deconvolving the 3D raw image by Wiener filer [39] along the $z$-direction pixel by pixel in the $x y$-plane [104].

The 1D deconvolution approach has the advantage of efficiently restoring the true image. However, deconvolution along the $z$-direction only is not sufficient. Besides, 1D deconvolution does not work well for large aperture objectives, because then the detection PSF strongly diverges along the lateral directions. Therefore, to recover a high- 
quality image, a full 3D deconvolution is required. Unfortunately, existing deconvolution methods with a single PSF cannot be directly used for light-sheet microscopy image reconstruction. As mentioned above, the imaging model of light-sheet microscopy does not match the convolution theorem as explicitly as in general deconvolution microscopy [106]. However, notice that the Airy beam illumination is quite structured along the $z$-direction and is only locally homogenous in the $x y$-plane; such features require a $3 \mathrm{D}$ deconvolution with a local PSF model. Based on this fact, I propose a blockby-block deconvolution approach to reconstruct images of light-sheet microscopy.

Several deconvolution algorithms have been proposed for 3D image deconvolution [107]. The well-known Richardson-Lucy deconvolution algorithm [41, 42], which assumes Poissonian noise in the measured images and estimates an object based on a MLE, is widely used in practice. It is indeed a very simple but powerful deconvolution technique. The advantages of the RL algorithm are that it is very easy to implement and intrinsically adapted to Poissonian noise which is very common in bio-imaging, and the non-negativity constraint, which is a very important constraint for image restoration, is already introduced implicitly. In addition, the RL algorithm is very suitable for volume image processing, because the core of the algorithm can be calculated by a Fast Fourier Transform (FFT) [108], which has big advantages for 3D image processing when using acceleration by GPUs [109]. The drawback of the RL algorithm is that noisy data can result in image artifacts, and that it converges very slowly. In practice, a regularization is usually introduced to avoid artifacts, and denoising is used in image deconvolution [110], which takes advantage of prior sample information. Based on this point of view, a modified RL algorithm with Total-Variation (TV) [111] regularization has been proposed [89]. Total Variation regularization is a very successful regularization technique, which results in sharp edges, high contrast, and a clear image, and it has been widely used to remove noise and to avoid ill-posed problems in image restoration [112]. As TV regularization imposes a penalty on first-order derivatives, RL deconvolution with Total Variation (RLTV) regularization is very suitable for noisy and blurred images that consist of numerous segment-flattened objects. However, there is a risk to introduce staircasing artifacts in continuous and smooth image restoration [113]. In reality, TV regularization is not always the best choice for smooth and continuous object restoration. For bio-imaging, a smoothening regularization would be more suitable, since biological samples are usually continuous and smooth.

Good's roughness penalty has been demonstrated to be a very good regularization for bio-imaging $[91,114]$. It has a very similar form as TV, but results in smooth and continuous images and outperforms many other regularization techniques in terms of meansquare-error [115]. Therefore, combining RL algorithm with roughness regularization is a very good alternative to RLTV to obtain smooth and continuous images. In this chapter, a 3D deconvolution approach with roughness regularization is proposed to reconstruct ALSM images. Volume data is divided into blocks and then each block is deconvolved with a local PSF. In the following sections, I firstly analyze the imaging model and derive the RLTV and RLRF deconvolution for ALSM. Then, I check the 
proposed algorithm by simulations and real sample image deconvolutions. Finally, I give a summary of my work.

\subsection{Airy Beam Light-Sheet Microscopy}

Experimentally, an Airy beam can be generated by modulating a Gaussian beam with a cubic phase mask, and then optically Fourier-transforming the beam with a lens. It has a structure as shown in figure 8.1.
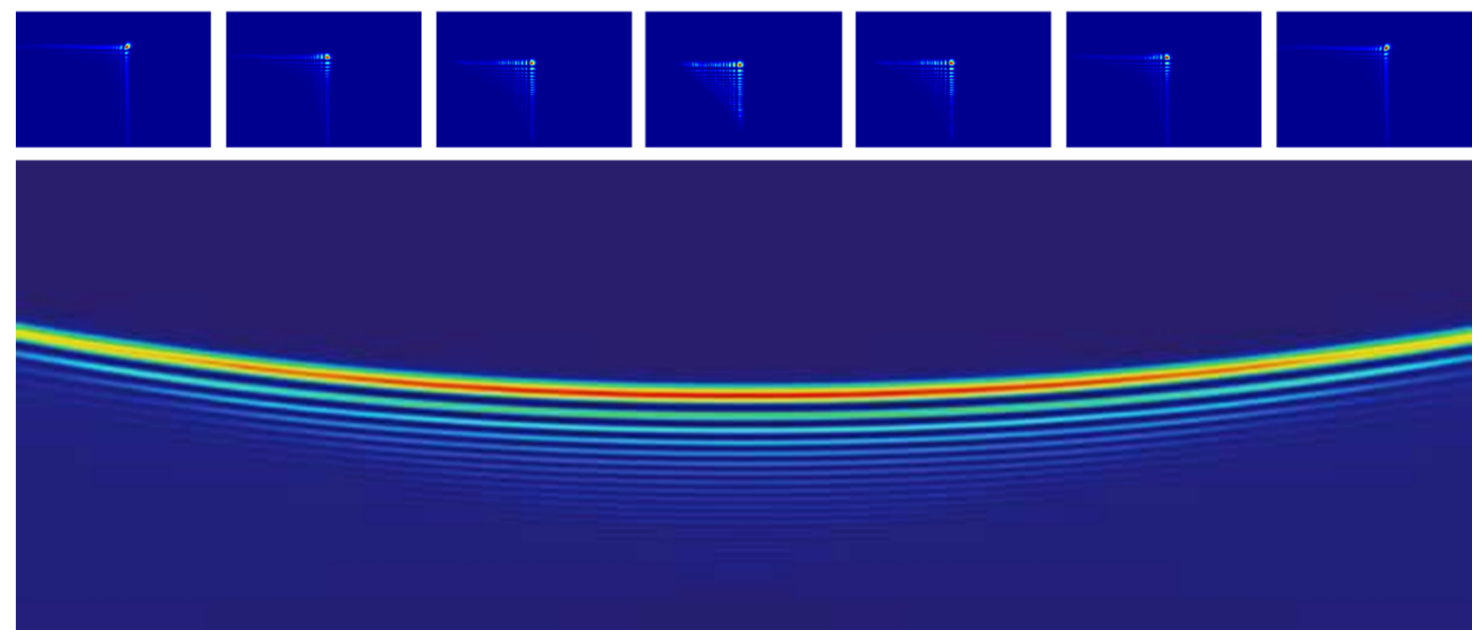

Figure 8.1 The structure of Airy beam. Upper: the transverse view in different x-position, bottom: $x-z$ view. The $x$ range (from left to right) spans $165 \mu \mathrm{m}$.

Their non-diffractive property makes Airy beams attractive for use in light-sheet microscopy, to obtain very large FOV images. The ALSM system consists of two arms, as shown in figure 8.2.

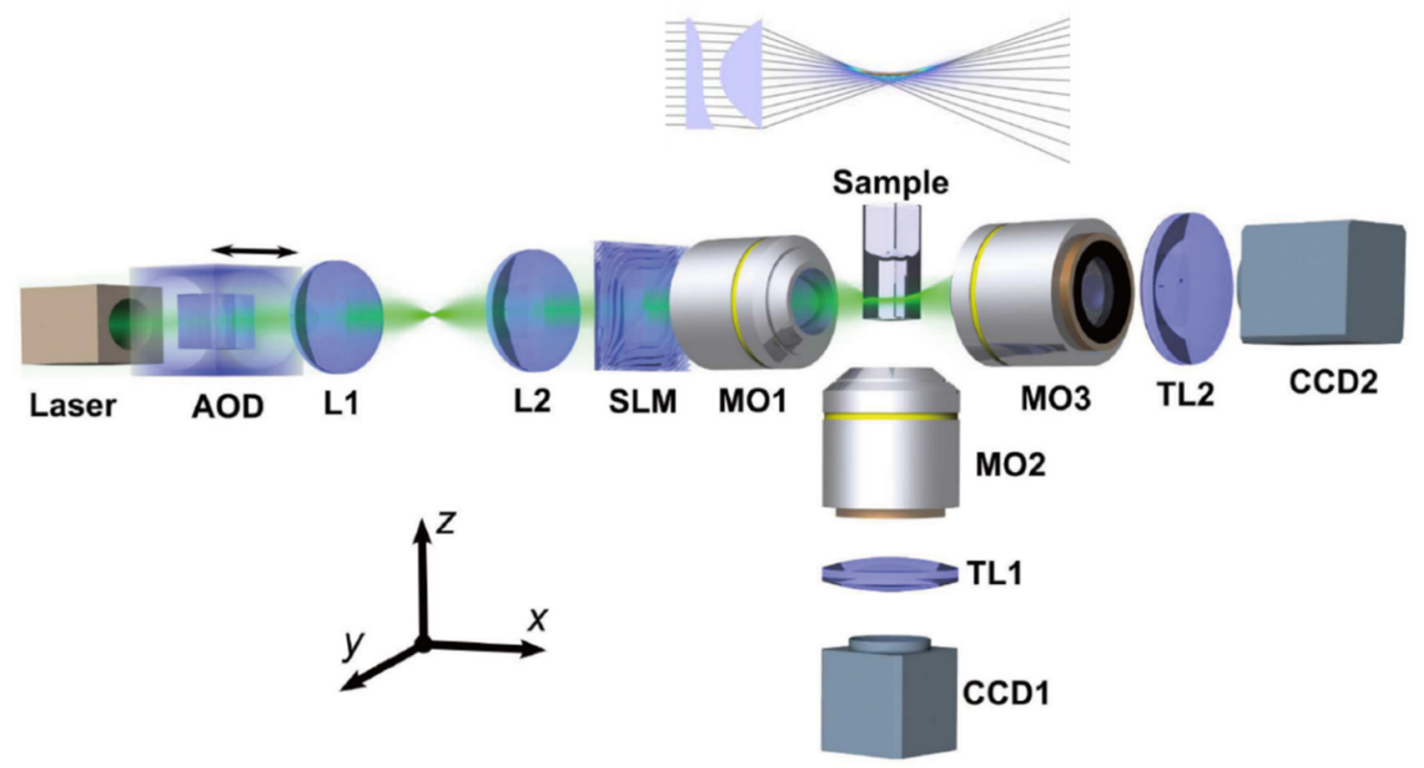

Figure 8.2 ALSM system [104]. 
In the horizontal arm, the Airy light sheet is generated by passing a laser beam (Laser, wavelength $488 \mathrm{~nm}$ or $532 \mathrm{~nm}$ ) through a fast digital scanning ( $y$-axis) acousto-optic deflector (AOD). Then, the beam goes through a cubic phase mask (SLM). Finally, it is resized by a proper telescope system (L1-L2) and projected onto the back aperture of the excitation objective (MO1), which Fourier-transforms the input light into the Airy beam. Not necessary but helpful are the detection objective (MO3), tube lens (TL2) and camera (CCD2) that are used for calibration of the light sheet microscope.

The sample is scanned by the AOD with the Airy light sheet, which is generated by the horizontal arm and propagates from the excitation objective (MO1) along the $x$-direction. In the vertical arm, the detection objective (MO2) and tube lens (TL1) are used to collect fluorescence excited by the Airy beam along the $z$-axis, and finally form an image on the camera (CCD1).

The coordinate system is defined as shown in figure 8.2. The Airy beam propagates along the $x$-direction and scans the sample along the $y$-direction. Beam scanning is realized by the AOD, which is synchronized with the camera. The sample and camera move together along the $z$-direction for $z$-sectioning.

Mathematically, the imaging function of a light-sheet microscope reads

$$
Y(x, y, z)=\iiint U\left(x-x^{\prime}, y-y^{\prime}, z-z^{\prime}\right) P\left(x^{\prime}, y^{\prime}, z^{\prime}-z\right) X\left(x^{\prime}, y^{\prime}, z^{\prime}\right) d x^{\prime} d y^{\prime} d z^{\prime}
$$

where $U(x, y, z)$ is the PSF of the imaging system, e.g. the PSF of a wide-field microscope, $P(x, y, z)$ denotes the illumination function caused by beam-scanning, $X(x, y, z)$ is the sample function, and $Y(x, y, z)$ is the image detected by the camera. Equation (8.1) shows that the imaging model is quite different from that of other imaging systems such as a wide-field or a confocal microscope, where it can be simply described by a convolution between the object function and the PSF of the imaging system. In light-sheet microscopy, the sample function is not only convolved by the PSF of the microscope in all dimensions, but also convolved with the illumination function along the $z$-direction. The convolution results in comet or flame artifacts in the measured image, which is similar to motion artifacts. To obtain the actual image, the raw image must be deconvolved. Even though ALSM is still a linear transformation system, it is challenging to deconvolve the raw image of ALSM in all dimensions. There is no final solution to this problem so far. However, if the illumination function $P(x, y, z)$ varies along the $z$-direction but is independent along the $x y$-diections, e.g. is constant in the $x y$-plane, the PSF then can be combined with the illumination function to create an equivalent PSF. I make use of this property of ALSM to derive the following deconvolution approach.

Beam scanning results in an illumination function that is equal to integrating the beam function over the scanning direction, which can be written as 


$$
P(x, z)=\int B(x, y, z) d y
$$

where $B(x, y, z)$ denotes the $3 \mathrm{D}$ beam function as figure 8.1 shows. The beam-scanning along the $y$-direction results in a parabolic iso-surface of a spatial structured illumination pattern, just as figure 8.3 shows.

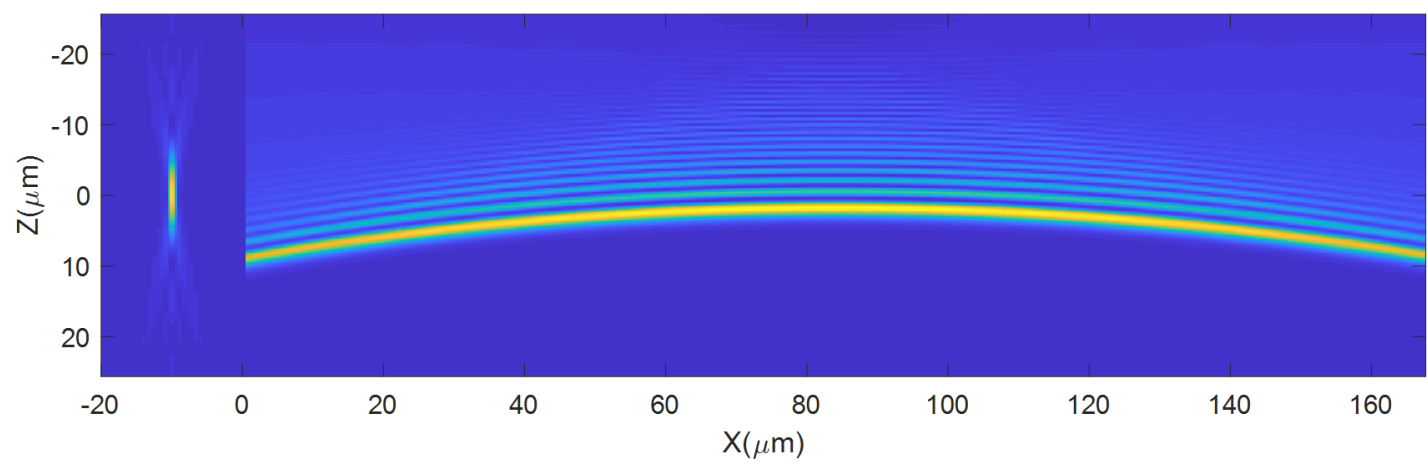

Figure 8.3 Illumination pattern by Airy beam scanning, left: Widefield PSF, right: Illumination pattern of Airy beam scanning. All images shown are in x-z plane at the center of the $y-$ range.

In a local area within a relatively small $x$-range, typically within $20 \mu \mathrm{m}$, the illumination pattern is quite structured along the $z$-direction and close to a plane in the $x y$-directions, which allows me to calculate a local PSF for ALSM by

$$
P S F(x, y, z)=U(x, y, z) \cdot P(x, z)
$$

With local PSFs, the raw image can be divided into several blocks along the $x$-direction, and then deconvolved block by block by conventional deconvolution algorithms. The PSF and the corresponding OTF at different $x$-positions is shown in figure 8.4.

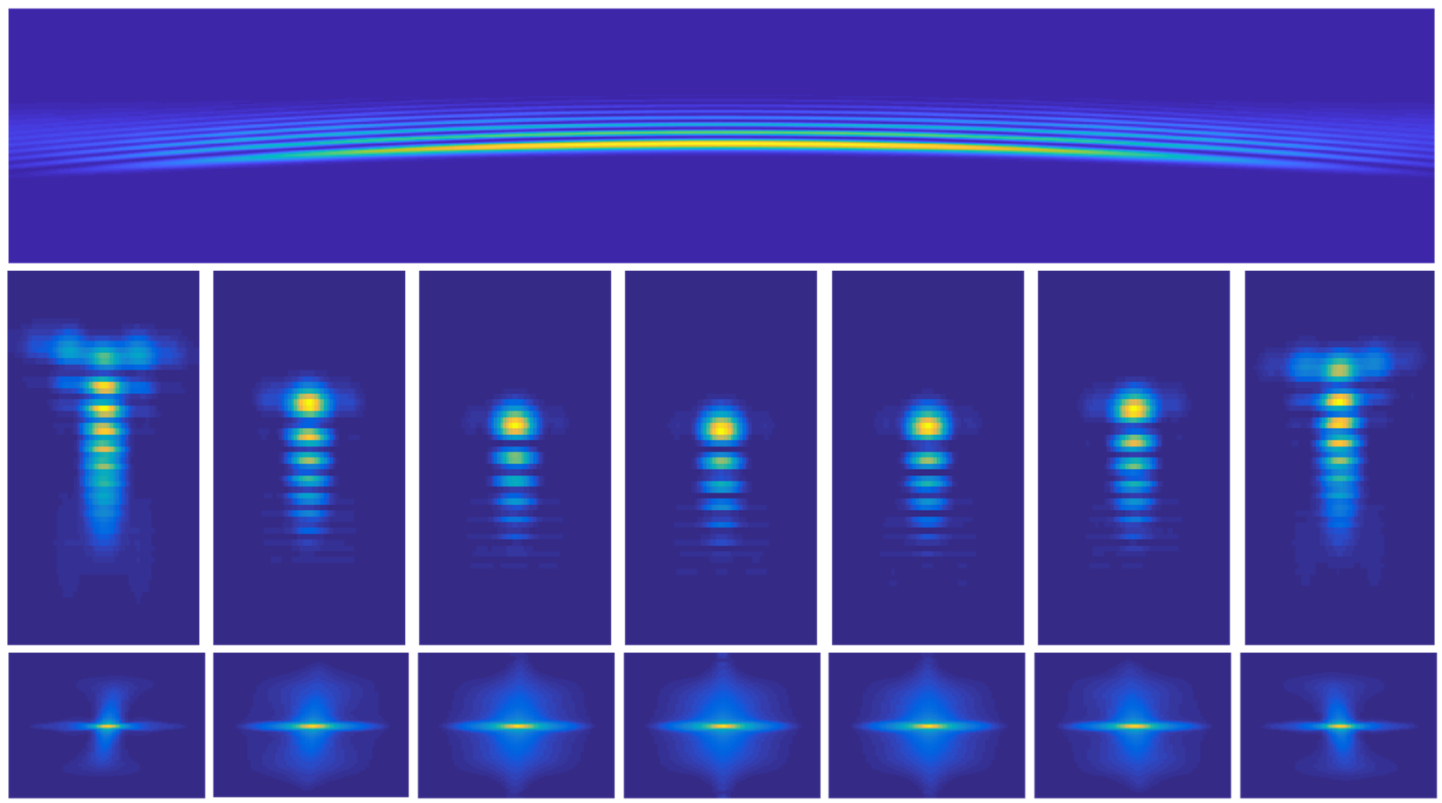

Figure 8.4 Upper: Illumination pattern of Airy beam scanning which is modulated by widefield PSF. Middle: The local PSFs in different x positions. Bottom: Corresponding OTF. All images shown are shown are the middle slice of $y$-range ( $x-z$ view). 
It can be seen from figure 8.4 that the local PSFs vary from block to block along the $x$ direction. However, it can be regarded as homogenous illumination in the $x y$-plane within short $x$-ranges. In practice, a very large range of the middle part can be cut off as a single block for deconvolution as the illumination pattern is perfectly homogeneous there. In contrast, the PSF on both side-blocks are quite different from the middle ones; the main lobe of the Airy beam is cut off and side lobes dominate. This, indeed, has a negative impact on the finally reconstructed image, as the support of the OTF is much narrower than of that in the middle. Nevertheless, the other parts still occupy a very large FOV with high image contrast. The block strategy does not necessarily have to be the same as shown in figure 8.4 , which will depend on the $x$-range of illumination homogeneity.

The block technique simplifies significantly the deconvolution as it divides a data volume into smaller blocks and makes it possible to employ parallel computing to accelerate $3 \mathrm{D}$ image reconstruction. Furthermore, each block can be de- convolved by a

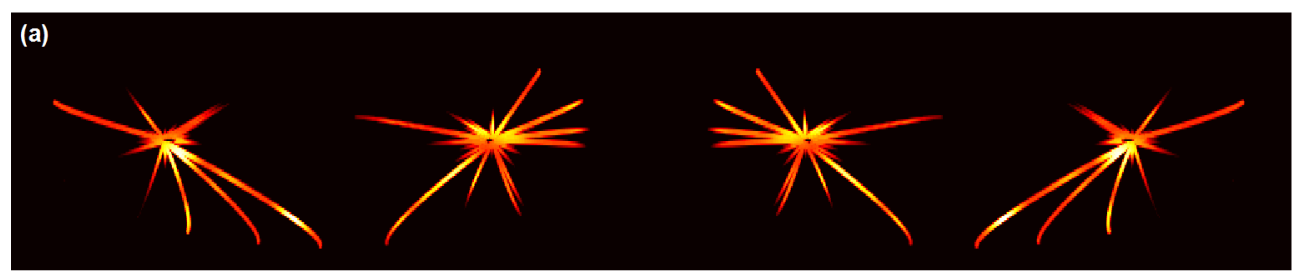

(b)

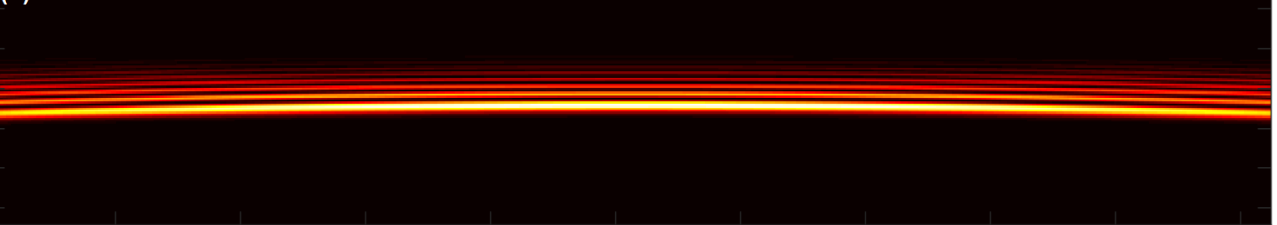

(c)

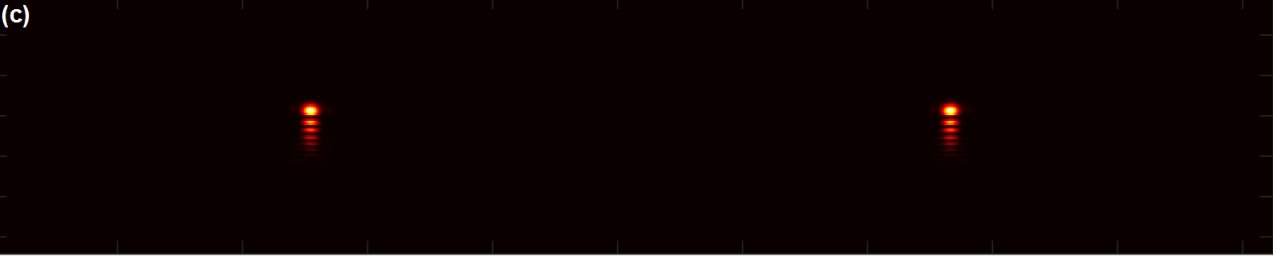

(d)
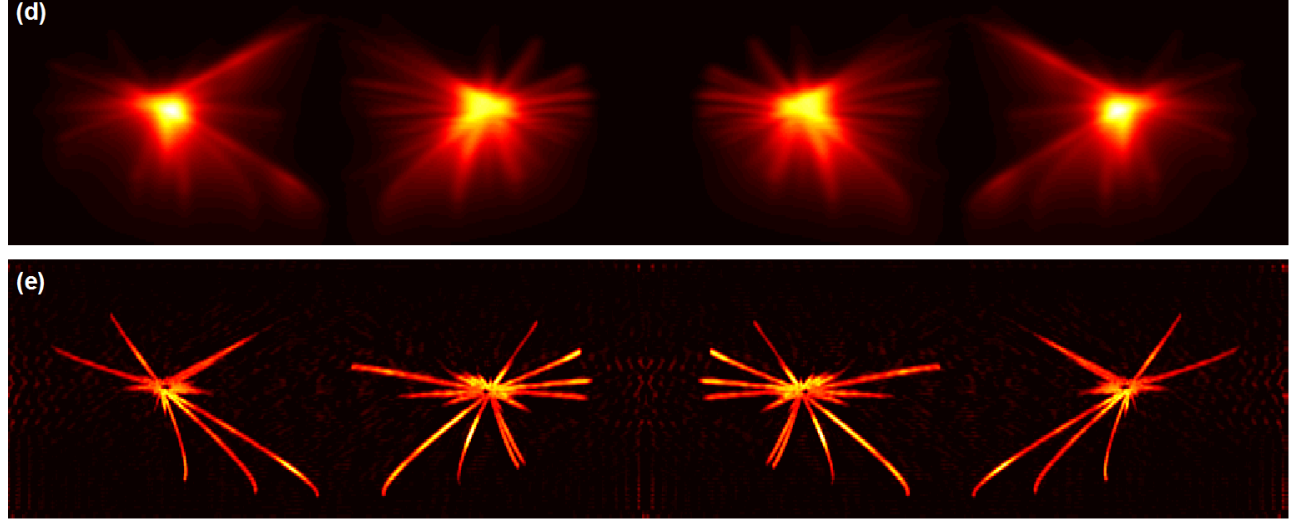

Figure 8.5 Deconvolution block-by-block. The blurred image is divided into two blocks and then deconvolved with local PSF by Wiener deconvolution. (a) The ground-truth, (b) illumination pattern (c) local PSF, (d) blurred image without noise, (e) deconvolved image. 
variety of existing deconvolution methods, such as Wiener or RL deconvolution. All this makes image reconstruction of ALSM image very easy. The feasibility of block-byblock deconvolution is demonstrated by the following simulations.

\subsection{Regularized Richardson-Lucy Deconvolution}

Poissonian noise is very common in imaging, based on which a MLE-based deconvolution can be derived. For a Poissonian noise image restoration model [116], the goal function is based on a MLE and can be written as

$$
E(f)=\sum_{i}[H * f-G \cdot \log (H * f)]_{i}
$$

where $G$ denotes the measured image, $H$ the PSF, $*$ is the convolution operator, and $\log$ denotes the natural logarithm. Let the gradient of the objective function be equal to zero, so that we have

$$
\frac{\partial E}{\partial f}=H * 1-H^{T} * \frac{G}{H * f}=0
$$

where $T$ denotes matrix transposition. Rearranging the above equation, we get

$$
1=H^{T} * \frac{G}{H * f}
$$

The goal function can minimized by using the following iteration [117]

$$
\frac{f_{k+1}}{f_{k}}=H^{T} * \frac{G}{H * f_{k}}
$$

Finally, RL deconvolution is done by

$$
f_{k+1}=f_{k} \cdot\left(H^{T} * \frac{G}{H * f_{k}}\right)
$$

where $f_{k}$ denotes the estimated object in the $k^{\text {th }}$ iteration. In practice, the core of the iteration formula can be calculated by a FFT, which is highly efficient for volume data processing, especially for 3D image deconvolution. Furthermore, acceleration of the RL algorithm is possible [118]. Just as many other deconvolution techniques, convergence of RL cannot be guaranteed, because noise could be amplified after a few number of iterations [89]. In practice, regularization is commonly used for artifact removal, denoising, and algorithm stabilization. Therefore, it is quite attractive to introduce regularization into RL to enhance its performance.

\subsubsection{Richardson-Lucy Deconvolution with TV regulariza- tion (RLTV)}

In image restoration, TV regularization is introduced to process very noisy data and to make the restoration algorithms result in very sharp and clear images. Total variation is defined by

$$
T V(f)=\int|\nabla f(X)| d X
$$

where $\nabla$ denotes the gradient operator. Usually, the gradient can be approximated by 
a discrete difference operator. Let us define the operator

$$
\left\{\begin{array}{l}
\nabla_{y} f_{i, j, k}=f_{i, j, k}-f_{i-1, j, k} \\
\nabla_{x} f_{i, j, k}=f_{i, j, k}-f_{i, j-1, k} \\
\nabla_{z} f_{i, j, k}=f_{i, j, k}-f_{i, j, k-1}
\end{array}\right.
$$

and the function

$$
\begin{gathered}
S(f)_{i, j, k}=\sqrt{\left(f_{i, j, k}-f_{i-1, j, k}\right)^{2}+\left(f_{i, j, k}-f_{i, j-1, k}\right)^{2}+\left(f_{i, j, k}-f_{i, j, k-1}\right)^{2}} \\
=\sqrt{\left|\nabla_{x} f_{i, j, k}\right|^{2}+\left|\nabla_{y} f_{i, j, k}\right|^{2}+\left|\nabla_{z} f_{i, j, k}\right|^{2}}
\end{gathered}
$$

where $i, j, k$ denote pixel indices along the $x, y, z$ directions, respectively. The total variation regularization function can then be represented as

$$
R_{T V}(f)=\sum_{i, j, k} S(f)_{i, j, k}
$$

The corresponding gradient can be derived as

$$
\nabla R_{T V}=\nabla_{x}^{*} \frac{\nabla_{x} f}{S(f)}+\nabla_{y}^{*} \frac{\nabla_{y} f}{S(f)}+\nabla_{z}^{*} \frac{\nabla_{z} f}{S(f)}
$$

where $\nabla_{x}^{*} f_{i, j, k}=x_{i, j, k}-x_{i+1, j, k}, \nabla_{y}^{*} f_{i, j, k}=x_{i, j, k}-x_{i, j+1, k}, \nabla_{z}^{*} f_{i, j, k}=x_{i, j, k}-x_{i, j, k+1}$. Similar to the derivation of the RL deconvolution algorithm based on a MLE, the iterative formula of RL deconvolution with TV regularization is found as

$$
f_{k+1}=\frac{f_{k}}{1+\lambda \nabla R_{T V}} \cdot\left(H^{T} * \frac{G}{H * f_{k}}\right)
$$

Compared to the formula of the RL algorithm, only one term is added, which does not increase the complexity of the algorithm but improves the performance of the RL algorithm significantly. In addition, the introduced term is quite easy to implement. Simulations show that it does not computationally affect the performance of the RL algorithm too much.

However, the problem of determining the value of the regularization parameter, an additional input parameter, which depends on the signal-to-noise ratio of the measured image, is involved. In practice, it is recommended to set it to around $10^{-3}$ [89]. Even though TV regularization preserves the sharp edges of an object and results in high contrast images, it can cause staircasing artifacts, as the gradient of the objective function is penalized. Therefore, tuning the regularization parameter is usually needed to restore good-quality images.

\subsubsection{Richardson-Lucy Deconvolution with Roughness Reg- ularization (RLRF)}

Usually, we hope to restore a continuous and smooth image of the object when smoothening regularization is introduced. Roughness regularization is very suitable for image restoration, as it is very similar to TV regularization and very easy to implement. I 
introduce here roughness regularization of RL to design a deconvolution algorithm for ALSM image reconstruction. Mathematically, the Roughness regularization function is defined by

$$
R_{R F}(f)=\int \frac{|\nabla f(X)|^{2}}{f(X)} d X
$$

where $X$ refers to any direction. Forward difference can be used to approximate the gradient, by which we can get its discrete form,

$$
R_{R F}^{X}(f)=\sum_{n} \frac{\left(f_{n+1}-f_{n}\right)^{2}}{f_{n}}
$$

The corresponding gradient

$$
\nabla_{X} R_{R F}=\frac{\partial R_{R F}^{X}}{\partial f_{n}}=-\frac{2\left(f_{n+1}-f_{n}\right)}{f_{n}}-\frac{\left(f_{n+1}-f_{n}\right)^{2}}{f_{n}^{2}}+\frac{2\left(f_{n}-f_{n-1}\right)}{f_{n-1}}
$$

In case of three-dimensional regularization, the Roughness regularization function can be represented as

$$
R_{R F}(f)=R_{R F}^{x}(f)+R_{R F}^{y}(f)+R_{R F}^{Z}(f)
$$

The corresponding gradient is

$$
\nabla R_{R F}=\nabla_{x} R_{R F}+\nabla_{y} R_{R F}+\nabla_{z} R_{R F}
$$

Similarly, the iteration formula of RLRF can be written as

$$
f_{k+1}=\frac{f_{k}}{1+\lambda \nabla R_{R F}} \cdot\left(H^{T} * \frac{G}{H * f_{k}}\right)
$$

Like RLTV, the term added into RLRF is quite simple, and only basic matrix operations are involved. Again, the calculation core, e.g. the term inside the bracket, can be calculated by FFT. Therefore, the above iterative algorithm is very easy to implement on any programming platform.

One may notice that the denominator in (17) could be zero, which will result in failure of calculation by a computer. Indeed, this is a real problem. Therefore, we can add a constraint to the algorithm, which is defined by

$$
f_{k+1}=\left\{\begin{array}{cc}
1, & f_{k}<1 \\
f_{k}, & f_{k} \geq 1
\end{array}\right.
$$

in each iteration. Setting the pixel-value of the goal function to one rather than zero will not affect the result of the deconvolution algorithm, since the pixel-value of an image is usually much larger than one. In reality, the pixel-value of an image is supposed to be non-negative or positive, therefore, it is a very useful constraint condition for image restoration [119]. 


\subsection{Results}

\subsubsection{Simulation of Noisy Image Deconvolution}

In order to verify the proposed methods, I firstly use an artificial 3D structure of microtubules [45] to simulate imaging and reconstruction of the original image by the proposed algorithm. The configuration of the computer used for these simulations is: i56600 CPU@3.3GHz, 32G RAM, 64-bit Windows 10 OS and the platform for programming and testing is based on Matlab R2017b.

The size of the original image is $256 \times 2048 \times 128$, and the pixels size (divided by image magnification) is $81.5 \mathrm{~nm}$ in the $x y$-plane and $400 \mathrm{~nm}$ in the $z$-direction. The 3D raw image is divided into 8 blocks with 256 pixels in $x$. Each block is deconvolved independently with a corresponding local 3D PSF, which is calculated using Equation (8.3). The 3D PSF of the microscope is calculated by a PSF Generator [120], an opensource plugin of ImageJ [121]. The Airy function is based on existing data. The blurred image is calculated using Equation (8.1), and Poissonian noise and background (=100) are added to the blurred image whose intensity range is between 0 and 2000 . The regularization parameter is set to $\lambda=10^{-3}$, and the number of iterations is set to 300 .
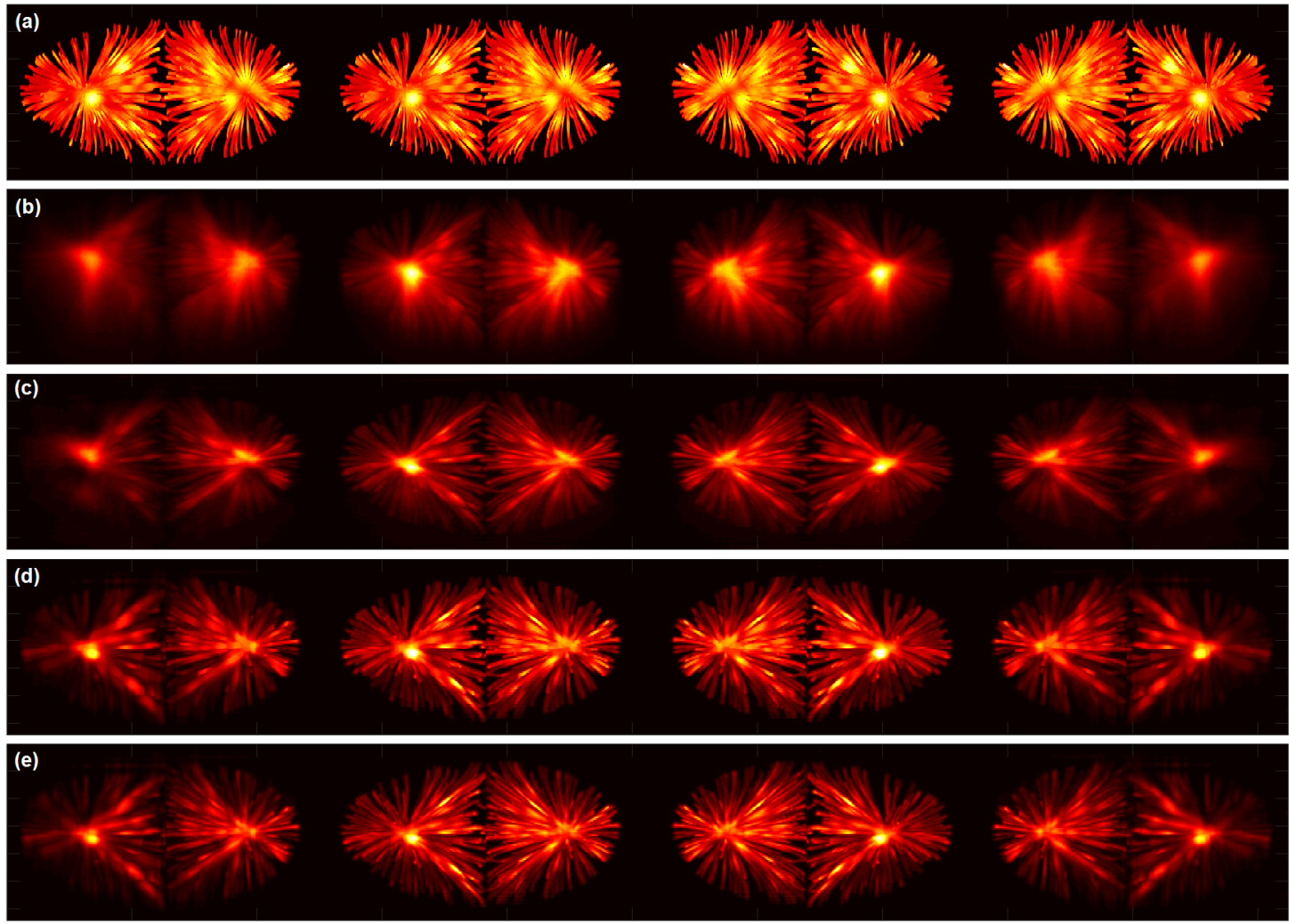

Figure 8.6 Light-sheet microscopy imaging and image reconstruction. (a) ground-truth, (b) blurred image with Poisson noise, (c) deconvolved image by 1D deconvolution, (d) deconvolved image by RL-TV algorithm, (d) deconvolved image by RL-RF algorithm. All images are visualized by maximum intensity projections (x-z view). 
All images are visualized by a maximum intensity projection. The PSF, Airy beam illumination and $x$-range here are completely the same as those shown in as figure 8.3. There are 4 pairs of twin microtubules, and they are put in different $x$-positions, so that we can compare the reconstructed image quality between different $x$-positions.

The simulation results show that the 3D deconvolution results in higher contrast and resolution compared to $1 \mathrm{D}$ deconvolution. The $1 \mathrm{D}$ deconvolved images are still very noisy and blurred, while the 3D deconvolved images are sharper and clearer. This implies that the regularization is very important for image denoising. The performance of RLRF and RLTV is very similar, both result in high quality images. However, RLRF is theoretically more suitable for continuous and smooth image restoration. This can be seen in the following experiments.

It also shows that the restored images in the middle of the $x$-range are quite good, while the most left and right images cannot be restored so well. The images degrade from $x<20 \mu \mathrm{m}$ and $x>140 \mu \mathrm{m}$ towards the boundary. The reason is that the PSF is not perfect due to the fact that the main lobe of the Airy beam is out of focus. In fact, the main lobe of the Airy beam has more impact on the quality of a restored image, because its frequency spectrum dominates the low frequency area in the Fourier domain, which is the main part of the support of a real image. Therefore, the use of the side parts (within about $20 \mu \mathrm{m}$ from the boundaries) should be avoided to guarantee a high quality image reconstruction.

\subsubsection{Deconvolution of Real Sample Image}

Based on the same platform, I also applied my algorithm to real sample 3D image deconvolution. The image data set was recorded by the ALSM set-up of the Dholakia group that has been used for multiple applications and which generates very good results [122]. The sample used for imaging are fluorescently labeled neurons in a cleared mouse-brain tissue section. The details of sample preparation can be found in Ref. [105].

A recorded raw image is shown in figure 8.6. The structure of the tissue is hardly recognizable. The total size of the sample is $88.7 \mu \mathrm{m} \times 83.5 \mu \mathrm{m} \times 128 \mu \mathrm{m}$, corresponding to an image size of $1088 \times 1024 \times 320$ voxels. Here, only the middle of the $x$-range with a suitable shape of the Airy beam was used. The existing method deconvolves the raw image along the $z$-direction using Wiener filtering and the simulated illumination function shown in figure 8.3.

For 3D deconvolution, the image data set is so large that it requires a proper sectioning strategy to perform the computations on a typical computer. In our experiment, the data set is not only divided into blocks along the $x$-direction, but also along the $y$-direction. In both directions, the data is divided into two equivalent blocks, 4 parts in total, but only two local PSFs are needed. This sectioning strategy significantly downsizes the data set for the deconvolution algorithm, by which less memory is required and parallel 
computing technology can be used to accelerate the image reconstruction. After deconvolution calculation, all de-convolved images are combined to restore the full 3D sample image.
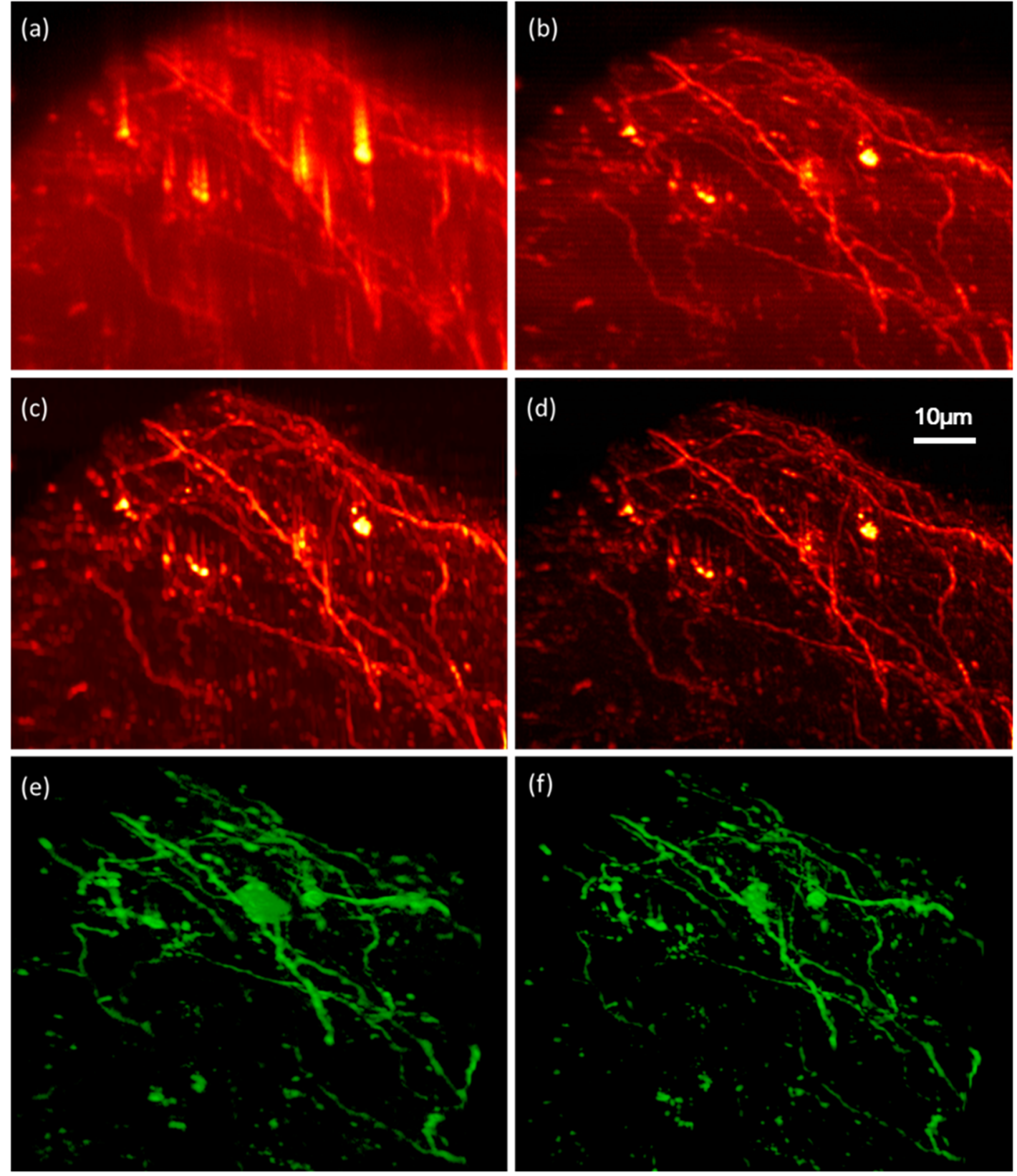

Figure 8.7 ALSM 3D sample image reconstructions, images (a d) are visualized by Maximum intensity projections (x-z view). (a) Recorded image, (b) 1D deconvolved image, (c) 3D deconvolved image by RLTV, (d) deconvolved image by RLRF, (e) 3D view of 1D deconvolved image, (f) 3D view of 3D image deconvolved by RLRF.

The results show that the $3 \mathrm{D}$ deconvolution results in higher contrast and resolution compared to $1 \mathrm{D}$ deconvolution. The $1 \mathrm{D}$ deconvolved image looks still very noisy and blurred. It is possible to improve the image quality by further deconvolution, however, estimating the correct PSF could be a problem. In contrast, the 3D deconvolved images look clearer and sharper, and more details are visible. 
The image deconvolved by RLTV shows very sharp edges and high contrast. However, it also shows obvious staircasing artifacts, which is not expected. In contrast, RLRF results in a smoother image, which looks more natural and clearer. By roughness penalty, the connected structures are better visible, while noise is significantly removed. Compared to TV regularization, roughness regularization is more suitable for bio-image restoration.

The deconvolution results for the real sample image match the results from the simulations very well. Both show the feasibility of the proposed deconvolution algorithm and demonstrate that the block-by-bock RL deconvolution with roughness regularization is a very promising approach for light-sheet microscopy image reconstruction.

\subsection{Discussion}

In this chapter, I proposed a block-by-block 3D deconvolution approach for image reconstruction in Airy-beam light-sheet microscopy. This microscopy is based on the structured illumination of an Airy beam. I employ the well-known Richardson-Lucy deconvolution with Good's roughness regularization to obtain smooth and continuous result. The simulation results show that the proposed approach outperforms the existing methods in reconstructing an ALSM image. Compared to 1D deconvolution reconstruction, the 3D deconvolution reconstruction results in higher contrast and resolution. Finally, I apply my algorithm to real sample image reconstruction. The experimental results show that it is a very promising approach for light-sheet microscopy image reconstruction. Actually, the proposed method is not only applicable to ALSM, but also to other light-sheet microscopy techniques, such as Bessel beam light-sheet microscopy whose illumination pattern is locally similar to Airy beam illumination. However, one problem still remains: the computation requires large amounts of memory and a highperformance computing platform. Therefore, acceleration of the proposed $3 \mathrm{D}$ deconvolution algorithm is necessary to realize fast $3 \mathrm{D}$ imaging. 


\section{Chapter 9}

\section{Discussion} and Outlook

In summary, the work presented in this thesis consists of three parts: Upgrading the Spinning Disk confocal ISM software package, deconvolution techniques for fluorescence microscopy, and efficient algorithm for L1-norm regularized optimization problems.

The SDC-ISM software package provides flexible functions and a friendly GUI for fast 3D confocal imaging, by which image acquisition can be done with a single click, as well as image reconstruction. This works is aimed to support any potential user who wants to upgrade and existing SDC system to SDC-ISM with super-resolution. It allows to convert the SDC system quickly, and to obtain high-quality images. In future work, deep learning methods could be considered for use ISM image reconstruction, which would not require the estimation of too many parameters that have to be tuned very carefully in the image reconstruction algorithm.

Deconvolution algorithms based on efficient non-linear optimization methods with regularizations, such as Total Variation regularization, Hessian regularization, roughness regularization, were developed for SOFI and ISM image enhancement and artifact removal. Furthermore, an accelerated regularized 3D Richardson-Lucy algorithm was developed for Airy light-sheet microscopy image reconstruction. The regularized 3D RL algorithm is very promising for processing 3D data acquired with modern high-speed and high-resolution imaging systems. In future work, it is very worth to try applying the proposed deconvolution method to large aperture objective Airy light-sheet microscopy, to which the 1D deconvolution does not work well any more. Meanwhile, the estimation and calculation of 3D PSFs for complex optical systems, such as SDC-ISM and ALSM, will become important for future applications.

L1-norm regularization is one important technique for sparse signal representation. The involved reconstruction algorithm is the key for applications in compressed sensing. I converted the very challenging L1-norm-regularized optimization problem to a normal non-linear optimization problem by approximating the L1-norm with a flexible smooth function. Then, the problem can be solved by existing and very powerful non-linear optimization methods, such as the LBFGS algorithm or the non-linear conjugate gradient methods. In future work, it would be worth to try out a novel technique, ADMM, which was developed to solve many kinds of complex optimization problem. 


\section{BIBLIOGRAPHY}

1. B. Herman and J. J. Lemasters, Optical microscopy: emerging methods and applications (Elsevier, 2012).

2. J. W. Lichtman and J.-A. Conchello, "Fluorescence microscopy," Nature Methods 2, 910-919 (2005).

3. M. J. Sanderson, I. Smith, I. Parker, and M. D. Bootman, "Fluorescence microscopy," Cold Spring Harbor protocols 2014, pdb.top071795-pdb.top071795 (2014).

4. M. Schrader, F. Meinecke, K. Bahlmann, M. Kroug, C. Cremer, E. Soini, and S. W. Hell, "Monitoring the excited state of a fluorophore in a microscope by stimulated emission," Bioimaging 3, 147-153 (1995).

5. P. T. Tran and F. Chang, "Transmitted Light Fluorescence Microscopy Revisited," The Biological Bulletin 201, 235-236 (2001).

6. J. Ries, S. Chiantia, and P. Schwille, "Accurate determination of membrane dynamics with linescan FCS," Biophysical journal 96, 1999-2008 (2009).

7. J. Pawley, Handbook of biological confocal microscopy (Springer Science \& Business Media, 2010).

8. R. Bracewell, "Convolution Theorem.," The Fourier Transform and Its Applications, 3rd ed. New York: McGraw-Hill, pp. 108-112, 1999.

9. J. Enderlein, "Advanced Fluorescence Microscopy" in: Comprehensive Biomedical Physics (ed. in chief A. Brahme), vol.4: Optical Molecular Imaging, eds. F. Alves and F. Kiessling, Elsevier, 2014, ch. 4.04, pp. 111-152.

10. J. Vangindertael, R. Camacho, W. Sempels, H. Mizuno, P. Dedecker, and K. P. F. Janssen, "An introduction to optical super-resolution microscopy for the adventurous biologist," Methods and Applications in Fluorescence 6, 022003 (2018).

11. S. W. Hell and J. Wichmann, "Breaking the diffraction resolution limit by stimulated emission: stimulated-emission-depletion fluorescence microscopy," Opt. Lett. 19, 780-782 (1994).

12. E. Betzig, G. H. Patterson, R. Sougrat, O. W. Lindwasser, S. Olenych, J. S. Bonifacino, M. W. Davidson, J. Lippincott-Schwartz, and H. F. Hess, "Imaging Intracellular Fluorescent Proteins at Nanometer Resolution," Science 313, 1642-1645 (2006).

13. X. Huang, J. Fan, L. Li, H. Liu, R. Wu, Y. Wu, L. Wei, H. Mao, A. Lal, P. Xi, L. Tang, Y. Zhang, Y. Liu, S. Tan, and L. Chen, "Fast, long-term, super-resolution imaging with Hessian structured illumination microscopy," Nature Biotechnology 36, 451 (2018).

14. J. Bewersdorf, A. Egner, and S. W. Hell, "4Pi-confocal microscopy is coming of age," GIT Imaging Microsc 4, 24-25 (2004).

15. S. W. Hell, E. H. K. Stelzer, S. Lindek, and C. Cremer, "Confocal microscopy with an increased detection aperture: type-B 4Pi confocal microscopy," Opt. Lett. 19, 222-224 (1994).

16. R. Heintzmann and T. Huser, "Super-resolution structured illumination microscopy," Chemical reviews 117, 13890-13908 (2017).

17. C. J. R. Sheppard, "Super-resolution in confocal imaging," Optik 80, 53-54 (1988).

18. C. B. Müller and J. Enderlein, "Image Scanning Microscopy," Physical Review Letters 104, 198101 (2010).

19. R. M. Power and J. Huisken, "A guide to light-sheet fluorescence microscopy for multiscale imaging," Nature Methods 14, 360 (2017). 
20. T. Dertinger, R. Colyer, R. Vogel, J. Enderlein, and S. Weiss, "Achieving increased resolution and more pixels with Superresolution Optical Fluctuation Imaging (SOFI)," Opt. Express 18, 1887518885 (2010).

21. S. Geissbuehler, C. Dellagiacoma, and T. Lasser, "Comparison between SOFI and STORM," Biomed. Opt. Express 2, 408-420 (2011).

22. T. Dertinger, R. Colyer, G. Iyer, S. Weiss, and J. Enderlein, "Fast, background-free, 3D superresolution optical fluctuation imaging (SOFI)," Proceedings of the National Academy of Sciences 106, 22287-22292 (2009).

23. T. Dertinger, J. Xu, O. F. Naini, R. Vogel, and S. Weiss, "SOFI-based 3D superresolution sectioning with a widefield microscope," Optical Nanoscopy 1, 2 (2012).

24. S. Geissbuehler, A. Sharipov, A. Godinat, N. L. Bocchio, P. A. Sandoz, A. Huss, N. A. Jensen, S. Jakobs, J. Enderlein, F. Gisou van der Goot, E. A. Dubikovskaya, T. Lasser, and M. Leutenegger, "Live-cell multiplane three-dimensional super-resolution optical fluctuation imaging," Nature Communications 5, 5830 (2014).

25. M. G. L. Gustafsson, "Surpassing the lateral resolution limit by a factor of two using structured illumination microscopy," Journal of Microscopy 198, 82-87 (2000).

26. M. G. L. Gustafsson, L. Shao, P. M. Carlton, C. J. R. Wang, I. N. Golubovskaya, W. Z. Cande, D. A. Agard, and J. W. Sedat, "Three-Dimensional Resolution Doubling in Wide-Field Fluorescence Microscopy by Structured Illumination," Biophysical Journal 94, 4957-4970 (2008).

27. R. Fiolka, L. Shao, E. H. Rego, M. W. Davidson, and M. G. L. Gustafsson, "Time-lapse two-color 3D imaging of live cells with doubled resolution using structured illumination," Proceedings of the National Academy of Sciences 109, 5311 (2012).

28. C. J. R. Sheppard, S. B. Mehta, and R. Heintzmann, "Superresolution by image scanning microscopy using pixel reassignment," Opt. Lett. 38, 2889-2892 (2013).

29. R. Tenne, U. Rossman, B. Rephael, Y. Israel, A. Krupinski-Ptaszek, R. Lapkiewicz, Y. Silberberg, and D. Oron, "Super-resolution enhancement by quantum image scanning microscopy," Nature Photonics 13, 116-122 (2019).

30. M. Castello, G. Tortarolo, M. Buttafava, T. Deguchi, F. Villa, S. Koho, L. Pesce, M. Oneto, S. Pelicci, L. Lanzanó, P. Bianchini, C. J. R. Sheppard, A. Diaspro, A. Tosi, and G. Vicidomini, "A robust and versatile platform for image scanning microscopy enabling super-resolution FLIM," Nature Methods 16, 175-178 (2019).

31. F. Strasser, M. Offterdinger, R. Piestun, and A. Jesacher, "Spectral image scanning microscopy," Biomed. Opt. Express 10, 2513-2527 (2019).

32. A. G. York, S. H. Parekh, D. D. Nogare, R. S. Fischer, K. Temprine, M. Mione, A. B. Chitnis, C. A. Combs, and H. Shroff, "Resolution doubling in live, multicellular organisms via multifocal structured illumination microscopy," Nature Methods 9, 749 (2012).

33. O. Schulz, C. Pieper, M. Clever, J. Pfaff, A. Ruhlandt, R. H. Kehlenbach, F. S. Wouters, J. Großhans, G. Bunt, and J. Enderlein, "Resolution doubling in fluorescence microscopy with confocal spinning-disk image scanning microscopy," Proceedings of the National Academy of Sciences 110, 21000-21005 (2013).

34. T. Azuma and T. Kei, "Super-resolution spinning-disk confocal microscopy using optical photon reassignment," Opt. Express 23, 15003-15011 (2015).

35. I. Gregor, M. Spiecker, R. Petrovsky, J. Großhans, R. Ros, and J. Enderlein, "Rapid nonlinear image scanning microscopy," Nature Methods 14, 1087 (2017). 
36. G. M. R. De Luca, R. M. P. Breedijk, R. A. J. Brandt, C. H. C. Zeelenberg, B. E. de Jong, W. Timmermans, L. N. Azar, R. A. Hoebe, S. Stallinga, and E. M. M. Manders, "Re-scan confocal microscopy: scanning twice for better resolution," Biomed. Opt. Express 4, 2644-2656 (2013).

37. O. Tzang, D. Feldkhun, A. Agrawal, A. Jesacher, and R. Piestun, "Two-photon PSF-engineered image scanning microscopy," Opt. Lett. 44, 895-898 (2019).

38. C. J. R. Sheppard, M. Buttafava, M. Castello, A. Diaspro, G. Tortarolo, A. Tosi, G. Vicidomini, and F. Villa, "Image scanning microscopy (ISM) with a single photon avalanche diode (SPAD) array detector," in (International Society for Optics and Photonics, 106790H.

39. R. C. Gonzalez and R. E. Woods, Digital Image Processing (Addison-Wesley Longman Publishing Co., Inc., 2001), p. 793.

40. W. H. F. Press, B. P.; Teukolsky, S. A.; and Vetterling, W. T, "Optimal (Wiener) Filtering with the FFT," Numerical Recipes in FORTRAN: The Art of Scientific Computing (1992).

41. L. B. Lucy, "An iterative technique for the rectification of observed distributions," The astronomical journal 79, 745 (1974).

42. W. H. Richardson, "Bayesian-Based Iterative Method of Image Restoration*," J. Opt. Soc. Am. 62, 55-59 (1972).

43. P. Getreuer, "Total variation deconvolution using split Bregman," Image Processing On Line 2, 158-174 (2012).

44. B.-J. Zandt, J. H. Liu, M. L. Veruki, and E. Hartveit, "All amacrine cells: quantitative reconstruction and morphometric analysis of electrophysiologically identified cells in live rat retinal slices imaged with multi-photon excitation microscopy," Brain Structure and Function 222, 151-182 (2017).

45. D. Sage, L. Donati, F. Soulez, D. Fortun, G. Schmit, A. Seitz, R. Guiet, C. Vonesch, and M. Unser, "DeconvolutionLab2: An open-source software for deconvolution microscopy," Methods 115, 28-41 (2017).

46. S. Geissbuehler, N. L. Bocchio, C. Dellagiacoma, C. Berclaz, M. Leutenegger, and T. Lasser, "Mapping molecular statistics with balanced super-resolution optical fluctuation imaging (bSOFI)," Optical Nanoscopy 1, 4 (2012).

47. J. K. Peter McCullagh, "Cumulants."

48. P. J.verveer, "Computational and Optical Methods for Improving Resolution and Signal Quality in Fluoresence Microscopy," PhD thesis.

49. A. Girsault, T. Lukes, A. Sharipov, S. Geissbuehler, M. Leutenegger, W. Vandenberg, P. Dedecker, J. Hofkens, and T. Lasser, "SOFI Simulation Tool: A Software Package for Simulating and Testing Super-Resolution Optical Fluctuation Imaging," PLOS ONE 11, e0161602 (2016).

50. S. C. Stein, A. Huss, D. Hähnel, I. Gregor, and J. Enderlein, "Fourier interpolation stochastic optical fluctuation imaging," Opt. Express 23, 16154-16163 (2015).

51. B. Matsumoto, Cell biological applications of confocal microscopy (Elsevier, 2003), Vol. 70.

52. S. Isbaner, "Extending Resolution in All Directions: Image Scanning Microscopy and Metalinduced Energy Transfer," PhD thesis (2019).

53. S. Palnitkar, Verilog HDL: a guide to digital design and synthesis (Pearson Education India, 2003).

54. J. Bergeron, Writing testbenches: functional verification of HDL models (Springer Science \& Business Media, 2012).

55. M. Graphics, "ModelSim SE User's Manual," Software Version 6(2006).

56. M. J. Rust, M. Bates, and X. Zhuang, "Sub-diffraction-limit imaging by stochastic optical 
reconstruction microscopy (STORM)," Nature Methods 3, 793 (2006).

57. P. Elena and Z. Michal, "Comparison of Algorithms For Fitting a Gaussian Function Used in Testing Smart Sensors," Journal of Electrical Engineering 66, 178-181 (2015).

58. M. Nord, P. E. Vullum, I. MacLaren, T. Tybell, and R. Holmestad, "Atomap: a new software tool for the automated analysis of atomic resolution images using two-dimensional Gaussian fitting," Advanced structural and chemical imaging 3, 9-9 (2017).

59. R. A. Caruana, R. B. Searle, T. Heller, and S. I. Shupack, "Fast algorithm for the resolution of spectra," Analytical Chemistry 58, 1162-1167 (1986).

60. H. Guo, "A Simple Algorithm for Fitting a Gaussian Function [DSP Tips and Tricks]," IEEE Signal Processing Magazine 28, 134-137 (2011).

61. S. Wolter, A. Löschberger, T. Holm, S. Aufmkolk, M.-C. Dabauvalle, S. van de Linde, and M. Sauer, "rapidSTORM: accurate, fast open-source software for localization microscopy," Nature Methods 9, 1040 (2012).

62. S. J. Miller, "The Method Of Least Squares," (2006).

63. R. W. Siregar, Tulus, and M. Ramli, "Analysis Local Convergence of Gauss-Newton Method," IOP Conference Series: Materials Science and Engineering 300, 012044 (2018).

64. K. Levenberg, "A method for the solution of certain non-linear problems in least squares," Quarterly of applied mathematics 2, 164-168 (1944).

65. D. Marquardt, "An Algorithm for Least-Squares Estimation of Nonlinear Parameters," Journal of the Society for Industrial and Applied Mathematics 11, 431-441 (1963).

66. G. Arfken, "The method of steepest descents," Mathematical methods for physicists 3, 428436 (1985).

67. E. W. Weisstein, "Gaussian Function," From MathWorld--A Wolfram Web Resource. http://mathworld.wolfram.com/GaussianFunction.html.

68. S. Boyd and L. Vandenberghe, Convex optimization (Cambridge university press, 2004).

69. R. Byrd, P. Lu, J. Nocedal, and C. Zhu, "A Limited Memory Algorithm for Bound Constrained Optimization," SIAM Journal on Scientific Computing 16, 1190-1208 (1995).

70. J. N. a. S. J. Wright, "Numerical Optimization," (2006).

71. L. Armijo, "Minimization of functions having Lipschitz continuous first partial derivatives," Pacific J. Math. 16, 1-3 (1966).

72. J. M. Guerra, "Super - resolution through illumination by diffraction - born evanescent waves," Applied physics letters 66, 3555-3557 (1995).

73. M. Müller, V. Mönkemöller, S. Hennig, W. Hübner, and T. Huser, "Open-source image reconstruction of super-resolution structured illumination microscopy data in ImageJ," Nature Communications 7, 10980 (2016).

74. T. Lukeš, P. Kř́žek, Z. Švindrych, J. Benda, M. Ovesný, K. Fliegel, M. Klíma, and G. M. Hagen, "Three-dimensional super-resolution structured illumination microscopy with maximum a posteriori probability image estimation," Opt. Express 22, 29805-29817 (2014).

75. N. Chakrova, B. Rieger, and S. Stallinga, "Deconvolution methods for structured illumination microscopy," J. Opt. Soc. Am. A 33, B12-B20 (2016).

76. J. Boulanger, N. Pustelnik, L. Condat, L. Sengmanivong, and T. Piolot, "Nonsmooth convex optimization for structured illumination microscopy image reconstruction," Inverse Problems 34, 095004 (2018).

77. A. Lal, C. Shan, and P. Xi, "Structured Illumination Microscopy Image Reconstruction 
Algorithm," IEEE Journal of Selected Topics in Quantum Electronics 22, 50-63 (2016).

78. E. Candes, and Justin Romberg., "I1-magic: Recovery of sparse signals via convex programming," URL: www. acm. caltech. edu/l1magic/downloads/l1magic.pdf, 14 (2005).

79. "Stable signal recovery from incomplete and inaccurate measurements," Communications on Pure and Applied Mathematics 59, 1207-1223 (2006).

80. W. Yin, S. Osher, D. Goldfarb, and J. Darbon, "Bregman Iterative Algorithms for L1-Minimization with Applications to Compressed Sensing," SIAM Journal on Imaging Sciences 1, 143-168 (2008).

81. T. Goldstein and S. Osher, "The Split Bregman Method for L1-Regularized Problems," SIAM Journal on Imaging Sciences 2, 323-343 (2009).

82. I. Selesnick, "Total variation denoising (an MM algorithm)," NYU Polytechnic School of Engineering Lecture Notes (2012).

83. G. Andrew and J. Gao, "Scalable training of L1-regularized log-linear models," in Proceedings of the 24th international conference on Machine learning, (ACM, 2007), 33-40.

84. E. J. Candes and M. B. Wakin, "An Introduction To Compressive Sampling," IEEE Signal Processing Magazine 25, 21-30 (2008).

85. R. G. Baraniuk, "Compressive Sensing [Lecture Notes]," IEEE Signal Processing Magazine 24, 118-121 (2007).

86. G. Strang, "Wavelet transforms versus Fourier transforms," Bulletin of the American Mathematical Society 28, 288-305 (1993).

87. J. M. Bioucas-Dias and M. A. T. Figueiredo, "A New TwIST: Two-Step Iterative Shrinkage/Thresholding Algorithms for Image Restoration," IEEE Transactions on Image Processing 16, 2992-3004 (2007).

88. I. Daubechies, M. Defrise, and C. De Mol, "An iterative thresholding algorithm for linear inverse problems with a sparsity constraint," Communications on Pure and Applied Mathematics: A Journal Issued by the Courant Institute of Mathematical Sciences 57, 1413-1457 (2004).

89. N. Dey, L. Blanc-Feraud, C. Zimmer, P. Roux, Z. Kam, J.-C. Olivo-Marin, and J. Zerubia, "Richardson-Lucy algorithm with total variation regularization for 3D confocal microscope deconvolution," Microscopy Research and Technique 69, 260-266 (2006).

90. S. Lefkimmiatis, A. Bourquard, and M. Unser, "Hessian-Based Norm Regularization for Image Restoration With Biomedical Applications," IEEE Transactions on Image Processing 21, 983-995 (2012).

91. S. Joshi and M. I. Miller, "Maximum a posteriori estimation with Good's roughness for threedimensional optical-sectioning microscopy," J. Opt. Soc. Am. A 10, 1078-1085 (1993).

92. G. M. P. v. Kempen, H. T. M. v. d. Voort, J. G. J. Bauman, and K. C. Strasters, "Comparing maximum likelihood estimation and constrained Tikhonov-Miller restoration," IEEE Engineering in Medicine and Biology Magazine 15, 76-83 (1996).

93. "https://commons.wikimedia.org/wiki/Category:Resolution_test_charts."

94. A. Girsault, T. Lukes, A. Sharipov, S. Geissbuehler, M. Leutenegger, W. Vandenberg, P. Dedecker, J. Hofkens, and T. Lasser, "SOFI Simulation Tool: A Software Package for Simulating and Testing Super-Resolution Optical Fluctuation Imaging," 11, 13 (2016).

95. O. E. Olarte, J. Andilla, E. J. Gualda, and P. Loza-Alvarez, "Light-sheet microscopy: a tutorial," Adv. Opt. Photon. 10, 111-179 (2018).

96. R. Tomer, K. Khairy, F. Amat, and P. J. Keller, "Quantitative high-speed imaging of entire 
developing embryos with simultaneous multiview light-sheet microscopy," Nature Methods $\mathbf{9}$, 755 (2012).

97. U. Krzic, S. Gunther, T. E. Saunders, S. J. Streichan, and L. Hufnagel, "Multiview light-sheet microscope for rapid in toto imaging," Nature Methods 9, 730 (2012).

98. J. Broky, G. A. Siviloglou, A. Dogariu, and D. N. Christodoulides, "Self-healing properties of optical Airy beams," Opt. Express 16, 12880-12891 (2008).

99. G. A. Siviloglou, J. Broky, A. Dogariu, and D. N. Christodoulides, "Observation of Accelerating Airy Beams," Physical Review Letters 99, 213901 (2007).

100. G. A. Siviloglou, J. Broky, A. Dogariu, and D. N. Christodoulides, "Ballistic dynamics of Airy beams," Opt. Lett. 33, 207-209 (2008).

101. J. Morris, T. Čižmár, H. Dalgarno, R. Marchington, F. Gunn-Moore, and K. Dholakia, "Realization of curved Bessel beams: propagation around obstructions," Journal of Optics 12, 124002 (2010).

102. J. Baumgartl, M. Mazilu, and K. Dholakia, "Optically mediated particle clearing using Airy wavepackets," Nature Photonics 2, 675 (2008).

103. S. Jia, J. C. Vaughan, and X. Zhuang, "Isotropic three-dimensional super-resolution imaging with a self-bending point spread function," Nature Photonics 8, 302 (2014).

104. T. Vettenburg, H. I. C. Dalgarno, J. Nylk, C. Coll-Lladó, D. E. K. Ferrier, T. Čižmár, F. J. Gunn-Moore, and K. Dholakia, "Light-sheet microscopy using an Airy beam," Nature Methods 11, 541 (2014).

105. J. Nylk, K. McCluskey, S. Aggarwal, J. A. Tello, and K. Dholakia, "Enhancement of image quality and imaging depth with Airy light-sheet microscopy in cleared and non-cleared neural tissue," Biomed. Opt. Express 7, 4021-4033 (2016).

106. J. G. McNally, T. Karpova, J. Cooper, and J. A. Conchello, "Three-Dimensional Imaging by Deconvolution Microscopy," Methods 19, 373-385 (1999).

107. G. M. P. Van Kempen, L. J. Van Vliet, P. J. Verveer, and H. T. M. Van Der Voort, "A quantitative comparison of image restoration methods for confocal microscopy," Journal of Microscopy 185, 354-365 (1997).

108. P. Duhamel and M. Vetterli, "Fast Fourier transforms: A tutorial review and a state of the art," pp. 259-299-259-299.

109. N. K. Govindaraju, B. Lloyd, Y. Dotsenko, B. Smith, and J. Manferdelli, "High performance discrete Fourier transforms on graphics processors," in SC '08: Proceedings of the 2008 ACM/IEEE Conference on Supercomputing, 2008), 1-12.

110. P. Sarder and A. Nehorai, "Deconvolution methods for 3-D fluorescence microscopy images," IEEE Signal Processing Magazine 23, 32-45 (2006).

111. L. I. Rudin and S. Osher, "Total variation based image restoration with free local constraints," in Proceedings of 1st International Conference on Image Processing, 1994), 31-35 vol.31.

112. S. Osher, M. Burger, D. Goldfarb, J. Xu, and W. Yin, "An Iterative Regularization Method for Total Variation-Based Image Restoration," Multiscale Modeling \& Simulation 4, 460-489 (2005).

113. T. Chan, A. Marquina, and P. Mulet, "High-Order Total Variation-Based Image Restoration," SIAM Journal on Scientific Computing 22, 503-516 (2000).

114. M. I. Miller and B. Roysam, "Bayesian image reconstruction for emission tomography incorporating Good's roughness prior on massively parallel processors," Proceedings of the National Academy of Sciences of the United States of America 88, 3223-3227 (1991).

115. P. J. Verveer and T. M. Jovin, "Image restoration based on Good's roughness penalty with 
application to fluorescence microscopy," J. Opt. Soc. Am. A 15, 1077-1083 (1998).

116. P. J. Verveer and T. M. Jovin, "Efficient superresolution restoration algorithms using maximum a posteriori estimations with application to fluorescence microscopy," J. Opt. Soc. Am. A 14, 1696-1706 (1997).

117. A. S. Carasso, "Linear and nonlinear image deblurring: A documented study," SIAM journal on numerical analysis 36, 1659-1689 (1999).

118. D. S. C. Biggs and M. Andrews, "Acceleration of iterative image restoration algorithms," Appl. Opt. 36, 1766-1775 (1997).

119. G. L. Zeng, "Gibbs artifact reduction by nonnegativity constraint," Journal of nuclear medicine technology 39, 213-219 (2011).

120. H. Kirshner, F. Aguet, D. Sage, and M. Unser, "3-D PSF fitting for fluorescence microscopy: implementation and localization application," Journal of Microscopy 249, 13-25 (2013).

121. T. J. Collins, "ImageJ for microscopy," BioTechniques 43, S25-S30 (2007).

122. J. Nylk, K. McCluskey, M. A. Preciado, M. Mazilu, Z. Yang, F. J. Gunn-Moore, S. Aggarwal, J. A. Tello, D. E. K. Ferrier, and K. Dholakia, "Light-sheet microscopy with attenuation-compensated propagation-invariant beams," Science Advances 4, eaar4817 (2018). 


\section{ACKNOWLEDGEMENTS}

This PhD project is supported by the European BE-Optical project and the GGNB programs of the Georg-August-Universität Göttingen. Firstly of all, I would like to thank my supervisor Prof. Dr. Jörg Enderlein, who offered me this great opportunity to pursue my $\mathrm{PhD}$ in the prestige Georg-August-Universität Göttingen three years' ago. In our eyes, Jörg is not just a supervisor, but also a friend. During the $\mathrm{PhD}$, Jörg guided me with his high enthusiasm and patient, by which I learned quickly and overcame the challenges in learning new things and solving complex physical and mathematical problems in my research topics. His helpful suggestion always let me had deeper insight to the problems I studied. What I really appreciate is that he supplied us enough freedom to do research in my work, which I think is the best way to foster strong research abilities. Together with Jörg, I would like to thank the other two thesis supervisors, Prof. Dr. Ulrich Parlitz and Dr. Michael Habeck, who gave a lot of helpful suggestions for my work and thesis. Without their guide, the work shown in this thesis could not be done.

I would like to thank Dr. Ingo Gregor and Sebastian Isbaner, together with whom I accomplished the SDC-ISM project. Our cooperation seems quite successful, even though there were so many unexpected problems emerged in the beginning. Ingo always happy to discuss with us any problem and help us to solve many unexpected technological problems. Sebastian built the setup and led me to do the experiments, by which many potential problems in the developed software were found; he is actually a very rigorous and efficient science researcher. I learned quite a lot from working with them. Without their help, many problems could not be solved.

I would like to thank Prof. Kishan Dholakia, the head of Optical Manipulation Group at the University of St Andrews, and Dr. Jonathan Nylk, who guided me to do the project of Airy-beam light-sheet microscopy image reconstruction during my 2 nd BEOptical secondment in their group last year.

I would like to thank Dr. Felix Koberling, the head of Systems Division of PicoQuant $\mathrm{GmbH}$, and Dr. Marcelle Koenig, who guided me to do the project of Correlation analysis of FCS during the my first BE-Optical secondment in PicoQuant. I also would like to thank Mariano Gonzalez Pisfil, who helped quite a lot in my work.

I would like to thank our sectaries and IT support, whose work guarantee the group running well. I would like to thank all the other group members in our group for their help during the period of my $\mathrm{PhD}$. In our group, we have many nice members from all over the world. People help each other and work in close cooperation to achieve good results and high-quality publications. Except research work, we also had a lot of fun together in many events such as conference travelling, barbecue, games, etc. In a word, I really enjoy working here. 



\title{
CURRICULUM VITAE
}

\author{
NAME : Shun Qin \\ TITLE : Mr. \\ NATIONALITY : China
}

\section{EDUCATION}

08.2016-present PhD candidate in Physics, Georg-August University Göttingen

Thesis: Advanced Image Deconvolution Techniques for Superresolution Microscopy

Supervisor: Prof. Dr. Jörg Enderlein

09.2011-03.2014 Master of Science in Electronic Science and Technology, Beijing Institute of Technology

Thesis: Research on Phase Retrieval Algorithm

Supervisor: Prof. Xinqi Hu

\section{PUBLICATIONS}

[1] Qin S, Hu X, Qin Q. Compressed sensing phase retrieval with phase diversity. OPT COMMUN. 2014 Jan 1;310:193-8.

[2] Shun Qin, Sebastian Isbaner, Ingo Gregor, Jörg Enderlein, Image Scanning Microscopy Upgrade Protocol: Achieving Double Resolution with a Standard Confocal Spinning-Disk (in preparation).

\section{CONFERENCES AND TALLKS}

\section{口 1st BE-Optical School, Göttingen, 2016}

Talk: Advanced Image Deconvolution Techniques for Super-resolution Microscopy

$\square$ PicoQuant 24th International Workshop on Single Molecule Spectroscopy and Super-resolution Microscopy in the Life Sciences, Berlin, 2017

Poster: Upgrade for Confocal Spinning Disk System to Spinning Disk Confocal Image canning Microscopy.

$\square$ The 1st Be-Optical workshop within the frame of the 3rd Biophysics by the Sea conference, Mallorca, Spain, 2017

Talk and poster: Spinning Disk Confocal Image Scanning Microscopy.

$\square$ 2nd Be-Optical Workshop, Montpellier, France, 2018

Talk: Introduction of Structured Illumination Microscopy (SIM) and its Application in Multi-plane Super Resolution Wide-field Microscopy.

$\square$ Be-Optical Final conference, Göttingen, Germany, 2019

Talk: Introduction of Maximum Likelihood Estimation and its Application in Data Analysis for Fluorescence Microscopy.

\section{SECONDMENTS}

$\square$ PicoQuant, Berlin, 2017

Project: Correlation Calculation and Visualization for Line Scanning FCS.

$\square$ University of St. Andrew, Prof. Kishan Dholakia's Group, St. Andrew, Scotland, 2018 Project: Airy Beam Light-sheet Microscopy Image Deconvolution. 
